\title{
Running in the ALPs
}

\author{
Mikael Chala ${ }^{1}$, Guilherme Guedes ${ }^{1,2, a}$, Maria $\operatorname{Ramos}^{1,2}$, Jose Santiago ${ }^{1}$ \\ ${ }^{1}$ CAFPE and Departamento de Física Teórica y del Cosmos, Universidad de Granada, Campus de Fuentenueva, 18071 Granada, Spain \\ ${ }^{2}$ Laboratório de Instrumentaçao e Física Experimental de Partículas, Departamento de Física da Universidade do Minho, Campus de Gualtar, \\ 4710-057 Braga, Portugal
}

Received: 29 December 2020 / Accepted: 7 February 2021 / Published online: 23 February 2021

(C) The Author(s) 2021

\begin{abstract}
The couplings of axion-like particles are probed by different experiments across a huge range of energy scales. Accordingly, a consistent analysis of the corresponding constraints requires the use of the renormalization group equations. We compute the full one-loop renormalization group evolution of all - relevant and marginal - parameters in the effective field theory for axion-like particles up to dimension five, above and below the electroweak scale, assuming only that new physics does not violate $\mathrm{CP}$. We also include a detailed discussion of the different bases used in the literature, the relations among them and the interplay of the CP and shift symmetries.
\end{abstract}

\section{Contents}

1 Introduction ................ 1

2 Effective field theory for ALPs . . . . . . . . . . 2

3 Divergences at one loop . . . . . . . . . . . . 4

3.1 Eliminating redundancy . . . . . . . . . 5

4 Anomalous dimensions and comparison with the literature ................ 6

5 Matching and running below the electroweak scale . 8

5.1 Divergences at one loop . . . . . . . . . . . 9 9

5.2 Eliminating redundancy . . . . . . . . . . . 12

5.3 Anomalous dimensions . . . . . . . . . . 13

6 Some phenomenological applications . . . . . . 15

7 Conclusions . . . . . . . . . . . . 16

Appendix A: One-loop diagrams in the ALP SMEFT . 17

Appendix B: Shift symmetry and relations between dif-

ferent bases . . . . . . . . . . . . . . . 21

Appendix C: Running of renormalizable parameters above the electroweak scale . . . . . . . . . . 22 Appendix D: One-loop diagrams in the ALP LEFT . . 22 References . . . . . . . . . . . . . . . 33

a e-mail: gguedes@lip.pt (corresponding author)

\section{Introduction}

Axion-like particles (ALPs), $s$, are pseudo-scalar singlets of the Standard Model (SM) gauge group, whose interactions are typically assumed to respect an approximate shift symmetry $s \rightarrow s+\sigma$ with constant $\sigma$. They arise naturally in theories with a spontaneously broken global symmetry such as the Peccei-Quinn solution to the strong CP problem [14], composite Higgs models [5-7] and others [8,9], as well as in different explanations for dark matter [10-12] and for the flavour [8,13-15] and hierarchy [16] problems. The shift symmetry must be broken explicitly by at least the ALP mass, and potentially also by its marginal couplings to the Higgs boson and even by higher-dimensional interactions. In the following we will consider a generic pseudo-scalar in which the shift symmetry is not imposed at any level. As we will discuss in detail below, it is easy to enforce shift invariance in the couplings we consider a posteriori.

The phenomenology of ALPs is mostly triggered by effective operators, the first of which arise at dimension five. Their impact has been studied at photon regeneration experiments [17-20], beam dumps [21,22] and high-energy colliders including LEP [23-25] and more recently the LHC [23-34] and future facilities [35-37]; see also Refs. [38-40]. Searches for ALPs produced from the blackbody photons in the solar core [41] and in other astrophysical events [42-49] have been performed too. ALP searches in flavour experiments have been studied recently in Refs. [50-55]; while CP violation signatures of ALPs have been considered in Ref. [56]. (Recent reviews on the physics of ALPs can be found for example in Refs. [57,58].)

These experiments span a huge range of energies, across which the Wilson coefficients of the ALP effective field theory (EFT) run, and mix, following the corresponding renormalization group equations (RGEs). Different computations of parts of the RGEs are spread in the literature $[24,59,60]$. However, to the best of our knowledge, there is no system- 
atic study of the entire ALP anomalous dimension matrix in any concrete basis of operators. We fill this gap in this work, extending also previous computations in different ways. In particular we compute the gauge dependence of the ALPfermion-fermion operators running, as well as the RGE dependence on the ALP-Higgs marginal coupling. Moreover, we work in a basis closer in spirit to the Warsaw basis [61] of the SMEFT; i.e. involving operators with less derivatives, not limiting to purely shift-invariant interactions. However, we cross check (and update where necessary) previous partial results performed with different sets of operators. Most importantly, we match at tree level at the electroweak (EW) scale the ALP EFT onto the low-energy version in which the heavy top quark, the Higgs and the $Z$ and $W$ gauge bosons are integrated out, and we compute the running within this ALP low-energy EFT (ALP LEFT) too, including the mixing of higher-dimensional operators into renormalizable ones, as well as the mixing between purely SM EFT operators and others that do involve the ALP. For the sake of generality, we compute this running for an arbitrary ALP LEFT, i.e. independently of whether the EFT above the EW scale is the ALP EFT or a more generic theory. As far as we are aware, essentially all results in this latter EFT are completely new.

The article is organised as follows. In Sect. 2 we introduce the ALP Lagrangian, including a Green basis of effective operators and their on-shell relations. We compute the one-loop counterterms for effective operators in Sect. 3. In Sect. 4 we obtain the complete anomalous dimension matrix for dimension-five operators at one loop. The different layers of the EFT valid at energies below the EW scale as well as their connection through renormalization and matching are discussed in Sect. 5. In Sect. 6 we present some phenomenological implications of the previous results, most importantly the possibility of probing ALP interactions to the $Z$ boson or to the top quark through their mixing into ALP-lepton operators. We conclude in Sect. 7. In Appendix A we provide the different Feynman diagrams computed for the renormalization of the ALP EFT. In Appendix B we report our results in a different basis commonly used too in phenomenological studies, while in Appendix C we collect the renormalization group running of renormalizable parameters within this EFT. Finally, in Appendix D we report the Feynman diagrams necessary for the computation of the RGEs in the ALP LEFT.

\section{Effective field theory for ALPs}

The renormalizable Lagrangian of the SM extended with a real pseudo-scalar singlet, $s$, reads,

$$
\begin{aligned}
& \mathcal{L}_{S M+s}=-\frac{1}{4} G_{\mu \nu}^{A} G_{A}^{\mu \nu}-\frac{1}{4} W_{\mu \nu}^{a} W_{a}^{\mu \nu}-\frac{1}{4} B_{\mu \nu} B^{\mu \nu} \\
& +\overline{q_{L}^{\alpha}} \mathrm{i} \not D q_{L}^{\alpha}+\overline{l_{L}^{\alpha}} \mathrm{i} \not D l_{L}^{\alpha}+\overline{u_{R}^{\alpha}} \mathrm{i} \not D u_{R}^{\alpha}+\overline{d_{R}^{\alpha}} \mathrm{i} \not D d_{R}^{\alpha}+\overline{e_{R}^{\alpha}} \mathrm{i} \not D e_{R}^{\alpha}
\end{aligned}
$$

$$
\begin{aligned}
& +\left(D_{\mu} \phi\right)^{\dagger}\left(D^{\mu} \phi\right)-\mu^{2}|\phi|^{2}-\lambda|\phi|^{4} \\
& -\left(y_{\alpha \beta}^{u} \overline{q_{L}^{\alpha}} \widetilde{\phi} u_{R}^{\beta}+y_{\alpha \beta}^{d} \overline{q_{L}^{\alpha}} \phi d_{R}^{\beta}+y_{\alpha \beta}^{e} \overline{l_{L}^{\alpha}} \phi e_{R}^{\beta}+\text { h.c. }\right) \\
& +\frac{1}{2}\left(\partial_{\mu} s\right)\left(\partial^{\mu} s\right)-\frac{1}{2} m^{2} s^{2}-\frac{\kappa_{s}}{3 !} s^{3} \\
& -\frac{\lambda_{s}}{4 !} s^{4}-\kappa_{s \phi} s|\phi|^{2}-\frac{\lambda_{s \phi}}{2} s^{2}|\phi|^{2}
\end{aligned}
$$

where $\alpha$ and $\beta$ are flavour indices, $q_{L}$ and $l_{L}$ denote the lefthanded (LH) quark and lepton doublets, respectively, and $u_{R}, d_{R}$ and $e_{R}$ the right-handed (RH) up-type, down-type quark and charged lepton singlets, respectively. The gluon and the EW gauge bosons are represented, as usual, by $G$ and by $W$ and $B$, respectively. The Higgs doublet is called $\phi$, while its conjugate is given by $\widetilde{\phi}=\epsilon \phi^{*}=\mathrm{i} \sigma_{2} \phi^{*}$, with $\sigma_{2}$ being the second Pauli matrix. A possible tadpole term has been eliminated via a field redefinition of $s$ and we use the minus-sign convention for the covariant derivative. In the renormalizable Lagrangian above, all coefficients are real except for the Yukawa couplings. Complex phases in these Yukawa couplings, as well as the couplings $\kappa_{s}$ and $\kappa_{s \phi}$, induce $\mathrm{CP}$ violation.

The first tower of effective interactions arise at dimension five. We provide a Green basis (checked with BasisGen [62]) of such set of operators in Table 1. Any other dimensionfive operator can be written in terms of these via algebraic or integration by parts identities. We have collected the operators in the table according to their $\mathrm{CP}$ properties, with $\mathrm{CP}$ even and $\mathrm{CP}$-odd operators in the top and bottom panels of the table, respectively. All the operators in the table are hermitian (for fixed values of flavour indices if present) and the corresponding Wilson coefficients are therefore real parameters (real matrices for operators involving flavour). A minimal basis of non-redundant operators, enough to describe all physical processes, is given by the operators named with $\mathcal{O}$. The ones denoted by $\mathcal{R}$ can be written in terms of the ones in the minimal basis by performing field redefinitions and are therefore equivalent to them in all physical observables. To $\mathcal{O}(1 / \Lambda)$ accuracy, these field redefinitions can be enforced through the equations of motion of the dimensionfour Lagrangian, namely,

$$
\begin{aligned}
\partial^{2} s= & -m^{2} s-\frac{\kappa_{s}}{2} s^{2}-\frac{\lambda_{s} s^{3}}{3 !}-\kappa_{s \phi}|\phi|^{2}-\lambda_{s \phi} s|\phi|^{2}, \\
D^{2} \phi_{k}= & -\mu^{2} \phi_{k}-2 \lambda|\phi|^{2} \phi_{k}-\kappa_{s \phi} s \phi_{k} \\
& -\frac{\lambda_{s \phi}}{2} s^{2} \phi_{k}-y_{\alpha \beta}^{u} \overline{q_{L j}^{\alpha}} \epsilon_{j k} u_{R}^{\beta} \\
& -y_{\alpha \beta}^{d *} \overline{d_{R}^{\beta}} q_{L k}^{\alpha}-y_{\alpha \beta}^{e *} e_{R}^{\beta} l_{L k}^{\alpha}, \\
i \not D q_{L k}^{\alpha}= & y_{\alpha \beta}^{d} \phi_{k} d_{R}^{\beta}+y_{\alpha \beta}^{u} \widetilde{\phi}_{k} u_{R}^{\beta}, \quad i \not D l_{L k}^{\alpha}=y_{\alpha \beta}^{e} \phi_{k} e_{R}^{\beta}, \\
i \not D u_{R}^{\alpha}= & y_{\beta \alpha}^{u *} \tilde{\phi}_{k}^{\dagger} q_{L k}^{\beta}, \quad i \not D d_{R}^{\alpha}=y_{\beta \alpha}^{d *} \phi_{k}^{\dagger} q_{L k}^{\beta}, \quad i \not D e_{R}^{\alpha}=y_{\beta \alpha}^{e *} \phi_{k}^{\dagger} l_{L k}^{\beta} ;
\end{aligned}
$$


where we use Latin indices for $S U$ (2). Using these equations we can arrive at the following identities, valid for physical observables:

$$
\begin{aligned}
& r_{s \phi \square} \mathcal{R}_{s \phi \square}=-r_{s \phi \square} \operatorname{Re}\left(y^{u}\right) \mathcal{O}_{s u \phi} \\
& +r_{s \phi \square} \operatorname{Re}\left(y^{d}\right) \mathcal{O}_{s d \phi}+r_{s \phi \square} \operatorname{Re}\left(y^{e}\right) \mathcal{O}_{s e \phi} \\
& +r_{s \phi \square} \operatorname{Im}\left(y^{u}\right) \mathcal{O}_{\text {su } \phi}
\end{aligned}
$$

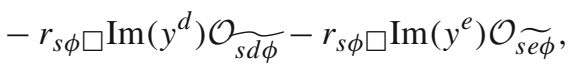

$$
\begin{aligned}
& r_{s q} \mathcal{R}_{s q}=-r_{s q} \operatorname{Re}\left(y^{u}\right) \mathcal{O}_{s u \phi}-r_{s q} \operatorname{Re}\left(y^{d}\right) \mathcal{O}_{s d \phi}
\end{aligned}
$$

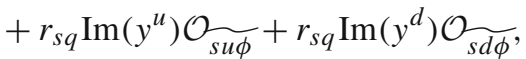

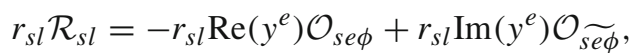

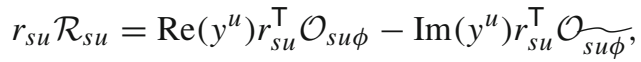

$$
\begin{aligned}
& r_{s d} \mathcal{R}_{s d}=\operatorname{Re}\left(y^{d}\right) r_{s d}^{\top} \mathcal{O}_{s d \phi}-\operatorname{Im}\left(y^{d}\right) r_{s d}^{\top} \mathcal{O}_{\text {sd }}, \\
& r_{s e} \mathcal{R}_{s e}=\operatorname{Re}\left(y^{e}\right) r_{s e}^{\top} \mathcal{O}_{s e \phi}-\operatorname{Im}\left(y^{e}\right) r_{s e}^{\top} \mathcal{O}_{\widetilde{s e \phi}} ;
\end{aligned}
$$

where flavour indices are left implicit and we have always assumed that each Wilson coefficient and its corresponding operator have the flavour indices in the same order so that, for instance,

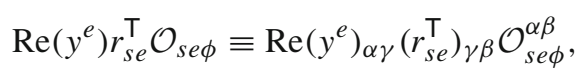

with repeated indices summed over.

For the $\mathrm{CP}$-odd ones we have

$$
\begin{aligned}
& r_{s \square} \mathcal{R}_{s} \square=-r_{s} \square m^{2} s^{3}-r_{s} \square \frac{\kappa_{s}}{2} s^{4}-r_{s} \square \frac{\lambda_{s}}{3 !} \mathcal{O}_{s} 5 \\
& -r_{s \square} \kappa_{s \phi} s^{2}|\phi|^{2}-r_{s \square} \square \lambda_{s \phi} \mathcal{O}_{s^{3}}, \\
& r_{s \phi \square} \mathcal{R} \widetilde{s \phi \square}=-2 r_{s \phi \square} \mu^{2} s|\phi|^{2}-4 r_{s \phi \square} \lambda \mathcal{O}_{s} \\
& -2 r \underset{s \phi \square}{\widetilde{N}} \kappa_{s \phi} s^{2}|\phi|^{2}-r_{s \phi \square} \lambda_{s \phi} \mathcal{O}_{s^{3}} \\
& -r_{\text {s } \square \square} \operatorname{Re}\left(y^{u}\right) \mathcal{O}_{\text {su } \phi}
\end{aligned}
$$

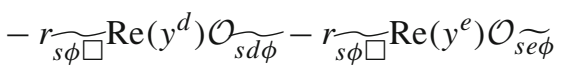

$$
\begin{aligned}
& -r_{s \phi \square} \operatorname{Im}\left(y^{u}\right) \mathcal{O}_{s u \phi}-r_{s \phi \square} \operatorname{Im}\left(y^{d}\right) \mathcal{O}_{s d \phi} \\
& -r_{s \phi \square} \operatorname{Im}\left(y^{e}\right) \mathcal{O}_{s e \phi}, \\
& r_{\phi s} \square \mathcal{R}_{\phi s \square}=-r_{\phi s \square} m^{2} s|\phi|^{2}-r_{\phi s} \square \frac{\kappa_{s}}{2} s^{2}|\phi|^{2} \\
& -r_{\phi s \square} \frac{\lambda_{s}}{3 !} \mathcal{O}_{s^{3}}-r_{\phi s \square} \kappa_{s \phi}|\phi|^{4}-\lambda_{s \phi} \mathcal{O}_{s}, \\
& r_{\tilde{s q}} \mathcal{R}_{\widetilde{s q}}=r_{\tilde{s q}} \operatorname{Re}\left(y^{u}\right) \mathcal{O}_{\tilde{s u \phi}}+r_{\widetilde{s q}} \operatorname{Re}\left(y^{d}\right) \mathcal{O}_{\widetilde{s d \phi}} \\
& +r_{\widetilde{s q}} \operatorname{Im}\left(y^{u}\right) \mathcal{O}_{s u \phi}+r_{\widetilde{s q}} \operatorname{Im}\left(y^{d}\right) \mathcal{O}_{s d \phi},
\end{aligned}
$$

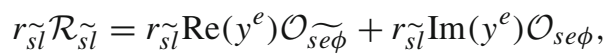

$$
\begin{aligned}
& r_{\widetilde{s u}} \mathcal{R}_{\widetilde{s u}}=\operatorname{Re}\left(y^{u}\right) r_{\widetilde{s u}}^{\top} \mathcal{O}_{\widetilde{s u \phi}}+\operatorname{Im}\left(y^{u}\right) r_{\widetilde{s u}}^{\top} \mathcal{O}_{s u \phi}, \\
& r_{\tilde{s d}} \mathcal{R}_{\tilde{s d}}=\operatorname{Re}\left(y^{d}\right) r_{\tilde{s d}}^{\top} \mathcal{O}_{s d \phi}+\operatorname{Im}\left(y^{d}\right) r_{\tilde{s d}}^{\top} \mathcal{O}_{s d \phi}, \\
& r_{\widetilde{s e}} \mathcal{R}_{\widetilde{s e}}=\operatorname{Re}\left(y^{e}\right) r_{\widetilde{s e}}^{\top} \mathcal{O}_{\widetilde{s e \phi}}+\operatorname{Im}\left(y^{e}\right) r_{\tilde{s d}}^{\top} \mathcal{O}_{s e \phi} .
\end{aligned}
$$

For the sake of generality we have not made use of the freedom to make $y^{e}$ and one of $y^{u}$ or $y^{d}$ diagonal, with real and positive entries, in the equations above.

Note that, despite their field content, the operators $\mathcal{R}_{s \phi \square}$ and $\mathcal{R}_{\widetilde{s \phi \square}}$ do not induce the process $s \phi \rightarrow \phi Z$. Indeed, by using the field redefinitions above we note that these operators are equivalent to Yukawa-like operators (or to operators with no gauge bosons in the CP-odd case), which clearly do not trigger the aforementioned process. An explicit calculation with these operators shows indeed that the corresponding amplitude goes with $p_{Z}^{2}$, which vanishes on-shell as the $Z$ boson is massless before EW symmetry breaking (EWSB). ${ }^{1}$

In the following we will consider that $\mathrm{CP}$ is a good symmetry of the EFT. This amounts to setting the coefficients of the CP-odd operators to zero, including $\kappa_{s}=\kappa_{s \phi}=0$ in the renormalizable Lagrangian and the ones in the bottom panel of Table 1. This is a radiatively stable choice up to the complex phase in the SM Yukawa couplings. The main goal of the present paper is to obtain the RGEs for the CP-even sector in isolation. However, we will provide our results for arbitrary Yukawa couplings, so that the mixing of the CPeven operators into the $\mathrm{CP}$-odd sector via the imaginary part of the SM Yukawa couplings can be easily obtained. Under these conditions, the relevant Lagrangian reads:

$$
\begin{aligned}
\mathcal{L}_{\mathrm{CP}-\mathrm{even}}= & \sum_{\psi=u, d, e} a_{s \psi \phi} \mathcal{O}_{s \psi \phi}+\sum_{X=G, W, B} a_{s \widetilde{X}} \mathcal{O}_{s \tilde{X}} \\
& +r_{s \phi \square} \mathcal{R}_{s \phi \square}+\sum_{\Psi=q, l, u, d, e} r_{s \Psi} \mathcal{R}_{s \Psi},
\end{aligned}
$$

where all the Wilson coefficients are real or real matrices in flavour space.

For the sake of generality we are not enforcing shift symmetry. However, in Appendix B we provide conditions on the Wilson coefficients that guarantee that this symmetry is preserved. We also explain why, although customary, trading the Yukawa-like operators $\mathcal{O}_{s \psi \phi}$, with $\psi=u, d, e$, by the explicitly shift-invariant terms $\partial_{\mu} s\left(\bar{\Psi} \gamma^{\mu} \Psi\right)$, with $\Psi=q_{L}, l_{L}, u_{R}, d_{R}, e_{R}[24,27,29,60,63]$, is not necessarily an optimal choice, as the set of operators thus constructed is overcomplete. Still, in the same appendix we will also provide the RGEs of these operators under some simplifying assumptions.

\footnotetext{
1 This can be trivially shown by considering the on-shell scattering amplitude $\mathcal{M}\left(1_{s} 2_{\phi} 3_{\phi} 4_{Z}\right)$. By dimensional arguments, $\mathcal{M} \sim\langle i j\rangle / \Lambda$ (or with squares). The only way to satisfy little-group covariance is therefore $i=j=4$, which makes $\mathcal{M}$ vanish due to the antisymmetry of brackets.
} 
Table 1 Green basis of effective operators of dimension five. All operators are hermitian (operators with flavour indices are hermitian for each fixed value of $\alpha$ and $\left.\beta,\left(\mathcal{O}_{\alpha \beta}\right)^{\dagger}=\mathcal{O}_{\alpha \beta}\right)$. The ones in the top (bottom) panel are $\mathrm{CP}$ conserving (violating). The dual field strength tensor is defined by $\widetilde{B}^{\mu \nu}=\frac{1}{2} \epsilon_{\mu \nu \rho \sigma} B^{\rho \sigma}$ (with $\epsilon^{0123}=1$ ) and likewise for $W$ and $G$

\begin{tabular}{|c|c|c|c|}
\hline Scalar & Yukawa & Derivative & Gauge \\
\hline & $\mathcal{O}_{s u \phi}^{\alpha \beta}=\mathrm{i} s\left(\overline{q_{L}^{\alpha}} \widetilde{\phi} u_{R}^{\beta}-\overline{u_{R}^{\beta}} \widetilde{\phi}^{\dagger} q_{L}^{\alpha}\right)$ & $\mathcal{R}_{s \phi \square}=\mathrm{i} s\left(\phi^{\dagger} D^{2} \phi-\left(D^{2} \phi\right)^{\dagger} \phi\right)$ & $\mathcal{O}_{s \widetilde{G}}=s G_{\mu \nu}^{A} \widetilde{G}_{A}^{\mu \nu}$ \\
\hline & $\mathcal{O}_{s d \phi}^{\alpha \beta}=\mathrm{i} s\left(\overline{q_{L}^{\alpha}} \phi d_{R}^{\beta}-\overline{d_{R}^{\beta}} \phi^{\dagger} q_{L}^{\alpha}\right)$ & $\mathcal{R}_{s q}^{\alpha \beta}=s\left(\overline{q_{L}^{\alpha}} \not D q_{L}^{\beta}+\overline{q_{L}^{\beta}} \overleftarrow{\not D} q_{L}^{\alpha}\right)$ & $\mathcal{O}_{s} \widetilde{W}=s W_{\mu \nu}^{a} \widetilde{W}_{a}^{\mu \nu}$ \\
\hline & $\mathcal{O}_{s e \phi}^{\alpha \beta}=\mathrm{is}\left(\overline{l_{L}^{\alpha}} \phi e_{R}^{\beta}-\overline{e_{R}^{\beta}} \phi^{\dagger} l_{L}^{\alpha}\right)$ & $\mathcal{R}_{s l}^{\alpha \beta}=s\left(\overline{l_{L}^{\alpha}} \not D l_{L}^{\beta}+\overline{l_{L}^{\beta}} \overleftarrow{\not D} l_{L}^{\alpha}\right)$ & $\mathcal{O}_{s \widetilde{B}}=s B_{\mu \nu} \widetilde{B}^{\mu \nu}$ \\
\hline & & $\mathcal{R}_{s u}^{\alpha \beta}=s\left(\overline{u_{R}^{\alpha}} \not D u_{R}^{\beta}+\overline{u_{R}^{\beta}} \overleftarrow{\not D} u_{R}^{\alpha}\right)$ & \\
\hline & & $\mathcal{R}_{s d}^{\alpha \beta}=s\left(\overline{d_{R}^{\alpha}} \not D d_{R}^{\beta}+\overline{d_{R}^{\beta}} \overleftarrow{D D} d_{R}^{\alpha}\right)$ & \\
\hline & & $\mathcal{R}_{s e}^{\alpha \beta}=s\left(\overline{e_{R}^{\alpha}} \not D e_{R}^{\beta}+\overline{e_{R}^{\beta}} \overleftarrow{\not D} e_{R}^{\alpha}\right)$ & \\
\hline $\mathcal{O}_{s^{5}}=s^{5}$ & $\mathcal{O}_{s u \phi}^{\alpha \beta}=s\left(\overline{q_{L}^{\alpha}} \widetilde{\phi} u_{R}^{\beta}+\overline{u_{R}^{\beta}} \widetilde{\phi}^{\dagger} q_{L}^{\alpha}\right)$ & $\mathcal{R}_{s \square}=s^{2} \partial_{\mu} \partial^{\mu} s$ & $\mathcal{O}_{s G}=s G_{\mu \nu}^{A} G_{A}^{\mu \nu}$ \\
\hline $\mathcal{O}_{s^{3}}=s^{3}|\phi|^{2}$ & $\mathcal{O}_{\frac{\alpha \beta}{\alpha \beta} \phi}^{\alpha \beta}=s\left(\overline{q_{L}^{\alpha}} \phi d_{R}^{\beta}+\overline{d_{R}^{\beta}} \phi^{\dagger} q_{L}^{\alpha}\right)$ & $\mathcal{R}_{\phi s \square}=|\phi|^{2} \partial^{2} s$ & $\mathcal{O}_{s W}=s W_{\mu \nu}^{a} W_{a}^{\mu \nu}$ \\
\hline \multirow[t]{6}{*}{$\mathcal{O}_{s}=s|\phi|^{4}$} & $\mathcal{O}_{\operatorname{se\phi }}^{\alpha \beta}=s\left(\overline{l_{L}^{\alpha}} \phi e_{R}^{\beta}+\overline{e_{R}^{\beta}} \phi^{\dagger} l_{L}^{\alpha}\right)$ & $\mathcal{R}_{\widetilde{s \phi \square}}=s\left(\phi^{\dagger} D^{2} \phi+\left(D^{2} \phi\right)^{\dagger} \phi\right)$ & $\mathcal{O}_{s B}=s B_{\mu \nu} B^{\mu \nu}$ \\
\hline & & $\mathcal{R}_{\widetilde{s q}}^{\alpha \beta}=s\left(\overline{q_{L}^{\alpha}} \mathrm{i} \not D q_{L}^{\beta}-\overline{q_{L}^{\beta}} \mathrm{i} \overleftarrow{\not D} q_{L}^{\alpha}\right)$ & \\
\hline & & $\mathcal{R}_{\widetilde{s l}}^{\alpha \beta}=s\left(\overline{l_{L}^{\alpha}} \mathrm{i} \not D l_{L}^{\beta}-\overline{l_{L}^{\beta}} \mathrm{i} \overleftarrow{D D} l_{L}^{\alpha}\right)$ & \\
\hline & & $\mathcal{R}_{\tilde{s u}}^{\alpha \beta}=s\left(\overline{u_{R}^{\alpha}} \mathrm{i} \not D u_{R}^{\beta}-\overline{u_{R}^{\beta}} \mathrm{i} \overleftarrow{\not D} u_{R}^{\alpha}\right)$ & \\
\hline & & $\mathcal{R}_{\widetilde{s d}}^{\alpha \beta}=s\left(\overline{d_{R}^{\alpha}} \mathrm{i} \not D d_{R}^{\beta}-\overline{d_{R}^{\beta}} \mathrm{i} \overleftarrow{\not D} d_{R}^{\alpha}\right)$ & \\
\hline & & $\mathcal{R}_{\tilde{s e}}^{\alpha \beta}=s\left(\overline{e_{R}^{\alpha}} \mathrm{i} \not D e_{R}^{\beta}-\overline{e_{R}^{\beta}} \mathrm{i} \overleftarrow{\not D} e_{R}^{\alpha}\right)$ & \\
\hline
\end{tabular}

\section{Divergences at one loop}

In order to obtain the RGEs of the ALP EFT, we have computed the divergences generated by one-particle-irreducible (1PI) diagrams at one loop with off-shell momenta and to order $\mathcal{O}(1 / \Lambda)$. In doing so, we have employed the background field method in the Feynman gauge in dimensional regularisation with space-time dimension $d=4-2 \epsilon$. The $1 / \epsilon$ poles obtained this way are gauge invariant. We have subsequently matched these onto the Green basis of operators of Table 1. We have implemented the model in FeynRules [64] and used FeynArts [65] and FormCalc [66] for the calculations. In a completely independent cross-check, we have evaluated by hand the Yukawa and $\lambda_{s \phi}$ pieces of each of the Feynman diagrams as obtained with Qgraf [67].

In the remainder of this section we will go through the different amplitudes that we need for matching the divergences in the ALP EFT. For each amplitude we will provide the ultraviolet (UV) divergence, matched onto our Green basis. We will denote the corresponding Wilson coefficients with a prime in order to distinguish them from the ones of the operator insertion in the one-loop calculation (which appear, without a prime, on the right-hand side of our equations). Recall that we assume the EFT to preserve $\mathrm{CP}$ and we are interested in the RGEs of the CP-even operators among themselves. In particular we will consider only insertions of CP-even oper- ators in the one-loop calculation. Within our assumptions the corresponding divergences can be again parameterized in terms of the CP-even operators up to the imaginary part of the SM Yukawa couplings. In this section we will provide the matching in the full basis, including the contribution via complex Yukawa couplings to the CP-odd operators so that the interested reader can obtain the corresponding mixing. In all these equations we leave flavour indices implicit.

- $s\left(p_{1}\right) \phi_{i}^{\dagger}\left(p_{2}\right) \rightarrow q_{L j}^{\alpha}\left(p_{3}\right) \overline{u_{R}^{\beta}}\left(p_{4}\right)$

The relevant diagrams, given in Fig. 1, produce the following UV divergence,

$$
\begin{aligned}
a_{\text {su } \phi}^{\prime} & -\mathrm{i} a_{\text {su } \phi}^{\prime} \\
= & -\frac{1}{(4 \pi)^{2} \epsilon}\left\{\left[\lambda_{s \phi}-\left(\frac{25 g_{1}^{2}}{36}+\frac{3 g_{2}^{2}}{4}+\frac{16 g_{3}^{2}}{3}\right)\right] a_{s u \phi}\right. \\
& \left.-y^{d} y^{d \dagger} a_{s u \phi}-a_{s d \phi} y^{d \dagger} y^{u}+y^{d} a_{s d \phi}^{\top} y^{u}\right\}
\end{aligned}
$$

- $s\left(p_{1}\right) \phi_{i}\left(p_{2}\right) \rightarrow q_{L j}^{\alpha}\left(p_{3}\right) \overline{d_{R}^{\beta}}\left(p_{4}\right)$

The relevant diagrams, given in Fig. 2, produce the following UV divergence,

$$
a_{s d \phi}^{\prime}-\mathrm{i} a_{s d \phi}^{\prime}
$$




$$
\begin{aligned}
= & -\frac{1}{(4 \pi)^{2} \epsilon}\left\{\left[\lambda_{s \phi}-\left(\frac{g_{1}^{2}}{36}+\frac{3 g_{2}^{2}}{4}+\frac{16 g_{3}^{2}}{3}\right)\right] a_{s d \phi}\right. \\
& \left.-y^{u} y^{u^{\dagger}} a_{s d \phi}-a_{s u \phi} y^{u^{\dagger}} y^{d}+y^{u} a_{s u \phi}^{\top} y^{d}\right\} .
\end{aligned}
$$

- $s\left(p_{1}\right) \phi_{i}\left(p_{2}\right) \rightarrow l_{L j}^{\alpha}\left(p_{3}\right) \overline{e_{R}^{\beta}}\left(p_{4}\right)$

The diagrams in Fig. 3 give,

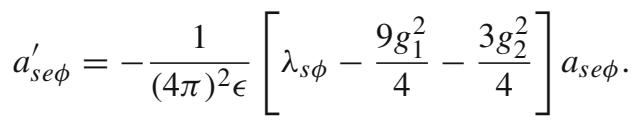

- $s\left(p_{1}\right) \rightarrow \phi_{i}\left(p_{2}\right) \phi_{j}^{\dagger}\left(p_{3}\right)$

The diagrams are shown in Fig. 4, and give

$$
\begin{aligned}
r_{s \phi \square}^{\prime}-\mathrm{i} r_{s \phi \square}^{\prime}= & -\frac{1}{16 \pi^{2} \epsilon}\left\{\operatorname{Tr}\left[y^{e} a_{s e \phi}^{\top}\right]\right. \\
& \left.+3 \operatorname{Tr}\left[y^{d} a_{s d \phi}^{\top}-a_{s u \phi} y^{u^{\dagger}}\right]\right\}, \\
r_{\phi s \square}^{\prime}= & 0 .
\end{aligned}
$$

- $s\left(p_{1}\right) \rightarrow \Psi^{\alpha}\left(p_{2}\right) \overline{\Psi^{\beta}}\left(p_{3}\right)$

For the process $s\left(p_{1}\right) \rightarrow \Psi^{\alpha}\left(p_{2}\right) \overline{\Psi^{\beta}}\left(p_{3}\right)$, with $\Psi=$ $q_{L}, l_{L}, u_{R}, d_{R}$ and $e_{R}$, we collect the one-loop diagrams in Figs. 5, 6, 7, 8, 9, respectively. The resulting divergences read ${ }^{2}$ :

$$
\begin{aligned}
r_{s q}^{\prime}+\mathrm{i} r_{s q}^{\prime}= & \frac{1}{32 \pi^{2} \epsilon}\left[a_{s u \phi} y^{u \dagger}+a_{s d \phi} y^{d \dagger}\right. \\
& \left.-\frac{g_{1}^{2}}{3} a_{s \widetilde{B}}-9 g_{2}^{2} a_{s \widetilde{W}}-16 g_{3}^{2} a_{s \widetilde{G}}\right], \\
r_{s l}^{\prime}+\mathrm{i} r_{\tilde{s l}}^{\prime}= & \frac{1}{32 \pi^{2} \epsilon}\left[a_{s e \phi} y^{e \dagger}-3 g_{1}^{2} a_{s \widetilde{B}}-9 g_{2}^{2} a_{s \widetilde{W}}\right], \\
r_{s u}^{\prime}+\mathrm{i} r_{s \widetilde{u}}^{\prime}= & -\frac{1}{16 \pi^{2} \epsilon}\left[a_{s u \phi}^{\top} y^{u}-\frac{8}{3} g_{1}^{2} a_{s \widetilde{B}}-8 g_{3}^{2} a_{s \widetilde{G}}\right] \\
r_{s d}^{\prime}+\mathrm{i} r_{\tilde{s d}}^{\prime}= & -\frac{1}{16 \pi^{2} \epsilon}\left[a_{s d \phi}^{\top} y^{d}-\frac{2}{3} g_{1}^{2} a_{s \widetilde{B}}-8 g_{3}^{2} a_{s \widetilde{G}}\right] \\
r_{s e}^{\prime}+\mathrm{i} r_{\tilde{s e}}^{\prime}= & -\frac{1}{16 \pi^{2} \epsilon}\left[a_{s e \phi}^{\top} y^{e}-6 g_{1}^{2} a_{s \widetilde{B}}\right] .
\end{aligned}
$$

We have partially cross-checked the results above by computing some amplitudes related to the ones above by gauge invariance. As an example the process $s B \rightarrow \bar{\Psi} \Psi$ allows us to cross check the anti-symmetric combination $\left(r_{s} \Psi\right)_{\alpha \beta}-\left(r_{s} \Psi\right)_{\beta \alpha}$.

${ }^{2}$ Note that only the terms proportional to the imaginary part of the Yukawa couplings contribute to the corresponding CP-odd operators.
- $s\left(p_{1}\right) \rightarrow V\left(p_{2}\right) V\left(p_{3}\right)$

No diagrams can be written at the order we are considering for the process $s \rightarrow B B$. The Feynman diagrams for the amplitudes $s \rightarrow W^{3} W^{3}$ and $s \rightarrow G G$ are shown in Figs. 10 and 11, respectively. The corresponding amplitudes are all non divergent. For $W$ bosons, the second diagram is zero while the divergences of all others together vanish. In the case of gluons, the second and third diagrams vanish, while the divergences of the rest of the diagrams cancel each other. It is evident from the diagrams that Yukawa-like operators do not renormalize ALP-vector-vector ones (redundant operators do not contribute to ALP-vector-vector couplings either). This is in agreement with the non-renormalization results in Refs. [68,69].

CP-even renormalizable couplings do not receive any contribution from dimension-five operators at one loop. This is easy to see from the fact that $\mathrm{CP}$-even renormalizable operators are even under $s \rightarrow-s$ whereas all dimension-five operators are odd under such replacement and therefore they cannot induce one-loop corrections to the renormalizable ones.

\subsection{Eliminating redundancy}

Once we have matched all the possible one-loop divergences onto our Green basis, we can use the relations in Eq. (11) to obtain the divergences in the minimal basis. From this point on, even though we will continue writing Yukawa couplings in matrix form, we will neglect their complex phases. This amounts to the following replacements:

$$
\begin{aligned}
a_{s u \phi}^{\prime} \rightarrow & a_{s u \phi}^{\prime}-r_{s \phi \square}^{\prime} y^{u}-r_{s q}^{\prime} y^{u}+y^{u} r_{s u}^{\prime \top} \\
= & \frac{-1}{(4 \pi)^{2} \epsilon}\left[\left(\lambda_{s \phi}-\frac{25 g_{1}^{2}}{36}-\frac{3 g_{2}^{2}}{4}-\frac{16 g_{3}^{2}}{3}\right) a_{s u \phi}\right. \\
& -y^{d} y^{d \dagger} a_{s u \phi}-a_{s d \phi} y^{d \dagger} y^{u}+y^{d} a_{s d \phi}^{\top} y^{u}+\frac{1}{2} a_{s u \phi} y^{u \dagger} y^{u} \\
& +\frac{1}{2} a_{s d \phi} y^{d \dagger} y^{u}+y^{u} y^{u \dagger} a_{s u \phi} \\
& -\operatorname{Tr}\left[y^{e} a_{s e \phi}^{\top}+3 y^{d} a_{s d \phi}^{\top}-3 a_{s u \phi} y^{u \dagger}\right] y^{u} \\
& \left.-\left(\frac{17}{6} g_{1}^{2} a_{s \widetilde{B}}+\frac{9}{2} g_{2}^{2} a_{s} \widetilde{W}+16 g_{3}^{2} a_{s} \widetilde{G}\right) y^{u}\right]
\end{aligned}
$$

$$
\begin{aligned}
a_{s d \phi}^{\prime} \rightarrow & a_{s d \phi}^{\prime}+r_{s \phi \square}^{\prime} y^{d}-r_{s q}^{\prime} y^{d}+y^{d} r_{s d}^{\prime \top} \\
= & \frac{-1}{(4 \pi)^{2} \epsilon}\left[\left(\lambda_{s \phi}-\frac{g_{1}^{2}}{36}-\frac{3 g_{2}^{2}}{4}-\frac{16 g_{3}^{2}}{3}\right) a_{s d \phi}\right. \\
& -y^{u} y^{u \dagger} a_{s d \phi}-a_{s u \phi} y^{u \dagger} y^{d} \\
& +y^{u} a_{s u \phi}^{\top} y^{d}+\frac{1}{2} a_{s u \phi} y^{u \dagger} y^{d}
\end{aligned}
$$




$$
\begin{aligned}
& +\frac{1}{2} a_{s d \phi} y^{d \dagger} y^{d}+y^{d} y^{d \dagger} a_{s d \phi} \\
& +\operatorname{Tr}\left[y^{e} a_{s e \phi}^{\top}+3 y^{d} a_{s d \phi}^{\top}-3 a_{s u \phi} y^{u \dagger}\right] y^{d} \\
& \left.-\left(\frac{5}{6} g_{1}^{2} a_{s \widetilde{B}}+\frac{9}{2} g_{2}^{2} a_{s \widetilde{W}}+16 g_{3}^{2} a_{s \widetilde{G}}\right) y^{d}\right], \\
a_{s e \phi}^{\prime} \rightarrow & a_{s e \phi}^{\prime}+r_{s \phi \square}^{\prime} y^{e}-r_{s l}^{\prime} y^{e}+y^{e} r_{s e}^{\prime \top} \\
= & \frac{-1}{(4 \pi)^{2} \epsilon}\left[\left(\lambda_{s \phi}-\frac{9 g_{1}^{2}}{4}-\frac{3 g_{2}^{2}}{4}\right) a_{s e \phi}+\frac{1}{2} a_{s e \phi} y^{e^{\dagger}} y^{e}\right. \\
& +y^{e} y^{e \dagger} a_{s e \phi}+\operatorname{Tr}\left[y^{e} a_{s e \phi}^{\top}+3 y^{d} a_{s d \phi}^{\top}-3 a_{s u \phi} y^{u \dagger}\right] y^{e} \\
& \left.-\left(\frac{15}{2} g_{1}^{2} a_{s \widetilde{B}}+\frac{9}{2} g_{2}^{2} a_{s} \tilde{W}\right) y^{e}\right] .
\end{aligned}
$$

\section{Anomalous dimensions and comparison with the literature}

In the previous section we have determined completely the divergent Lagrangian in the physical basis as

$\mathcal{L}_{\text {div }}=\mathcal{O}_{n} a_{n}^{\prime} \equiv \mathcal{O}_{n} \frac{\mathcal{C}_{n m}}{32 \pi^{2} \epsilon} a_{m}$,

where $n, m$ run over all operators (including flavour indices when present) and the coefficients $\mathcal{C}_{n m}$ involve only dimensionfour couplings. The $\beta$-function governing the RGEs is given by

$\beta_{a_{n}}=16 \pi^{2} \mu \frac{d a_{n}}{d \mu}=\gamma_{n m} a_{m}$,

where $\gamma$ is the anomalous dimension matrix. It is completely determined by the divergence matrix $\mathcal{C}$ up to the wave function renormalization factor for the different operators:

$\gamma_{n m}=-\left(\mathcal{C}_{n m}+K_{n}^{F} \delta_{n m}\right)$,

where $K^{F}$ parametrises the divergences in the wave function renormalization factors of each operator:

$Z_{n}^{F}=1+\frac{K_{n}^{F}}{32 \pi^{2} \epsilon}$,

with

$$
\begin{aligned}
& Z_{\mathcal{O}_{s u \phi}}^{F}=\sqrt{Z_{q_{L}} Z_{\phi} Z_{u_{R}}}, \quad Z_{\mathcal{O}_{s} \widetilde{G}}^{F}=\sqrt{Z_{G}}, \\
& Z_{\mathcal{O}_{s d \phi}}^{F}=\sqrt{Z_{q_{L}} Z_{\phi} Z_{d_{R}}}, \quad Z_{\mathcal{O}_{s \tilde{W}}}^{F}=\sqrt{Z_{W}}, \\
& Z_{\mathcal{O}_{\text {sed }}}^{F}=\sqrt{Z_{l_{L}} Z_{\phi} Z_{e_{R}}}, \quad Z_{\mathcal{O}_{s \tilde{B}}}^{F}=\sqrt{Z_{B}},
\end{aligned}
$$

and the following wave function renormalization factors, in agreement with Refs. [70,71], ${ }^{3}$

$$
\begin{aligned}
& Z_{q_{L}}=1-\frac{1}{96 \pi^{2} \epsilon}\left[\frac{1}{6} g_{1}^{2}+\frac{9}{2} g_{2}^{2}+8 g_{3}^{2}+3 y^{u} y^{u \dagger}+3 y^{d} y^{d \dagger}\right] \\
& Z_{l_{L}}=1-\frac{1}{64 \pi^{2} \epsilon}\left[g_{1}^{2}+3 g_{2}^{2}+2 y^{e} y^{e \dagger}\right] \\
& Z_{u_{R}}=1-\frac{1}{48 \pi^{2} \epsilon}\left[\frac{4}{3} g_{1}^{2}+4 g_{3}^{2}+3 y^{u \dagger} y^{u}\right] \\
& Z_{d_{R}}=1-\frac{1}{48 \pi^{2} \epsilon}\left[\frac{1}{3} g_{1}^{2}+4 g_{3}^{2}+3 y^{d \dagger} y^{d}\right] \\
& Z_{e_{R}}=1-\frac{1}{16 \pi^{2} \epsilon}\left[g_{1}^{2}+y^{e \dagger} y^{e}\right] \\
& Z_{\phi}=1+\frac{1}{32 \pi^{2} \epsilon}\left[g_{1}^{2}+3 g_{2}^{2}-2 \gamma_{\phi}^{(Y)}\right] \\
& Z_{B}=1-\frac{41 g_{1}^{2}}{96 \pi^{2} \epsilon} \\
& Z_{W}=1+\frac{19 g_{2}^{2}}{96 \pi^{2} \epsilon} \\
& Z_{G}=1+\frac{14 g_{3}^{2}}{32 \pi^{2} \epsilon}
\end{aligned}
$$

where we have defined

$\gamma_{\phi}^{(Y)} \equiv \operatorname{Tr}\left[y^{e \dagger} y^{e}+3 y^{u \dagger} y^{u}+3 y^{d \dagger} y^{d}\right]$.

The final result for the $\beta$-functions, written as usual in matrix form with flavour indices implicit, reads:

$$
\begin{aligned}
\beta_{a_{s u \phi}=} & 2\left[\left(\lambda_{s \phi}-\frac{17 g_{1}^{2}}{24}-\frac{9 g_{2}^{2}}{8}-4 g_{3}^{2}+\frac{1}{2} \gamma_{\phi}^{(Y)}\right) a_{s u \phi}\right. \\
& -\frac{3}{4} y^{d} y^{d \dagger} a_{s u \phi}+\frac{5}{4} y^{u} y^{u \dagger} a_{s u \phi}+a_{s u \phi} y^{u \dagger} y^{u} \\
& +y^{d} a_{s d \phi}^{\top} y^{u}-\frac{1}{2} a_{s d \phi} y^{d \dagger} y^{u} \\
& -\left(\frac{17 g_{1}^{2}}{6} a_{s \widetilde{B}}+\frac{9 g_{2}^{2}}{2} a_{s} \widetilde{W}+16 g_{3}^{2} a_{s} \widetilde{G}\right. \\
& \left.\left.+\operatorname{Tr}\left[y^{e} a_{s e \phi}^{\top}+3 y^{d} a_{s d \phi}^{\top}-3 a_{s u \phi} y^{u \dagger}\right]\right) y^{u}\right] \\
\beta_{a_{s d \phi}}= & 2\left[\left(\lambda_{s \phi}-\frac{5 g_{1}^{2}}{24}-\frac{9 g_{2}^{2}}{8}-4 g_{3}^{2}+\frac{1}{2} \gamma_{\phi}^{(Y)}\right) a_{s d \phi}\right. \\
& -\frac{3}{4} y^{u} y^{u \dagger} a_{s d \phi}+\frac{5}{4} y^{d} y^{d \dagger} a_{s d \phi}+a_{s d \phi} y^{d \dagger} y^{d} \\
& +y^{u} a_{s u \phi}^{\top} y^{d}-\frac{1}{2} a_{s u \phi} y^{u^{\dagger}} y^{d} \\
& -\left(\frac{5 g_{1}^{2}}{6} a_{s \widetilde{B}}+\frac{9 g_{2}^{2}}{2} a_{s} \widetilde{W}+16 g_{3}^{2} a_{s} \widetilde{G}\right.
\end{aligned}
$$

${ }^{3}$ Note however that in Ref. [70] the Higgs is also split into background and quantum fields, therefore comparing $Z_{\phi}$ in this case is not straightforward. It can be also trivially seen that $Z_{s}$ vanishes. 


$$
\begin{aligned}
& \left.\left.-\operatorname{Tr}\left[y^{e} a_{s e \phi}^{\top}+3 y^{d} a_{s d \phi}^{\top}-3 a_{s u \phi} y^{u \dagger}\right]\right) y^{d}\right], \\
\beta_{a_{s e \phi}}= & 2\left[a_{s e \phi}\left(\lambda_{s \phi}-\frac{15 g_{1}^{2}}{8}-\frac{9 g_{2}^{2}}{8}+\frac{1}{2} \gamma_{\phi}^{(Y)}\right)\right. \\
& +\frac{5}{4} y^{e} y^{e \dagger} a_{s e \phi}+a_{s e \phi} y^{e \dagger} y^{e} \\
& -\left(\frac{15 g_{1}^{2}}{2} a_{s \widetilde{B}}+\frac{9 g_{2}^{2}}{2} a_{s \widetilde{W}}\right. \\
& \left.\left.-\operatorname{Tr}\left[y^{e} a_{s e \phi}^{\top}+3 y^{d} a_{s d \phi}^{\top}-3 a_{s u \phi} y^{u \dagger}\right]\right) y^{e}\right], \\
\beta_{a_{s \widetilde{B}}}= & \frac{41}{3} g_{1}^{2} a_{s \widetilde{B}}, \\
\beta_{a_{s} \widetilde{W}}= & -\frac{19}{3} g_{2}^{2} a_{s \widetilde{W}}, \\
\beta_{a_{s \widetilde{G}}}= & -14 g_{3}^{2} a_{s \widetilde{G}} .
\end{aligned}
$$

A more graphic picture of the operator mixing can be obtained for the case in which different fermion families factorize, so that all dimension-five Wilson coefficients are flavour diagonal, $a_{\alpha \beta}=\delta_{\alpha \beta} a_{\alpha}$ (and neglecting also offdiagonal Yukawa couplings). Writing $\gamma_{n m}$, where $n$ runs over $\mathcal{O}_{s u \phi}^{\alpha}, \mathcal{O}_{s d \phi}^{\alpha}, \mathcal{O}_{s e \phi}^{\alpha}, \mathcal{O}_{s \widetilde{G}}, \mathcal{O}_{s \widetilde{W}}$ and $\mathcal{O}_{s \widetilde{B}}$, and $m$ over the same operators but with flavour index $\rho$, we can express the anomalous dimensions in the following form:
Note that, due to the contribution to $r_{s \phi \square}$, even in the flavour diagonal case there is inter-generational mixing but the choice of diagonal Wilson coefficients is radiatively stable (up to the small non-diagonal terms in the SM Yukawa couplings).

Different pieces of the anomalous dimension matrix have been previously computed in the literature. In particular, the mixing of the operators $\mathcal{O}_{s u \phi}, \mathcal{O}_{s d \phi}$ and $\mathcal{O}_{s e \phi}$ driven by Yukawa interactions has been obtained in Ref. [60]. Such work relies, however, on a different basis of effective interactions, where the fermionic operators take the form $\left(\partial_{\mu} s\right) \bar{\Psi} C_{\Psi} \gamma^{\mu} \Psi$. Using the relation between their Wilson coefficients and $a_{s u \phi}, a_{s d \phi}$ and $a_{s e \phi}$ we have obtained the beta functions for the latter from the results in Ref. [60] and compared them with our direct calculation reported in Eqs. (51)-(53). The results completely agree up to a sign difference in the terms proportional to $\operatorname{Tr}\left[y^{e} a_{s e \phi}^{\top}+3 y^{d} a_{s d \phi}^{\top}-3 a_{s u \phi} y^{u^{\dagger}}\right] y^{\psi}$, with $\psi=u, d, e$. Unfortunately, we do not find enough information to track the origin of this discrepancy.

It is also worth emphasizing that the set of effective operators used in Ref. [60] is over-complete. As we discuss in detail in Appendix B, if the Yukawa couplings commute with $C_{\Psi}$, a minimal explicitly shift-invariant basis involves only $\mathrm{RH}$ currents. The RGEs in this new basis read:

$\gamma=\left(\begin{array}{cccccc}\gamma_{11}+6 y_{u}^{\alpha} y_{u}^{\rho} & y_{d}^{\alpha} y_{u}^{\alpha}-6 y_{u}^{\alpha} y_{d}^{\rho} & -2 y_{u}^{\alpha} y_{e}^{\rho} & -32 g_{3}^{2} y_{u}^{\alpha} & -9 g_{2}^{2} y_{u}^{\alpha} & -\frac{17}{3} g_{1}^{2} y_{u}^{\alpha} \\ y_{u}^{\alpha} y_{d}^{\alpha}-6 y_{d}^{\alpha} y_{u}^{\rho} & \gamma_{22}+6 y_{d}^{\alpha} y_{d}^{\rho} & 2 y_{d}^{\alpha} y_{e}^{\rho} & -32 g_{3}^{2} y_{d}^{\alpha} & -9 g_{2}^{2} y_{d}^{\alpha} & -\frac{5}{3} g_{1}^{2} y_{d}^{\alpha} \\ -6 y_{e}^{\alpha} y_{u}^{\rho} & 6 y_{e}^{\alpha} y_{d}^{\rho} & \gamma_{33}+2 y_{e}^{\alpha} y_{e}^{\rho} & 0 & -9 g_{2}^{2} y_{e}^{\alpha} & -15 g_{1}^{2} y_{e}^{\alpha} \\ 0 & 0 & 0 & -14 g_{3}^{2} & 0 & 0 \\ 0 & 0 & 0 & 0 & -\frac{19}{3} g_{2}^{2} & 0 \\ 0 & 0 & 0 & 0 & 0 & \frac{41}{3} g_{1}^{2}\end{array}\right)$,

where a $\delta_{\alpha \rho}$ should be understood in every entry in which the $\rho$-index does not explicitly appear, and we have defined

$$
\begin{aligned}
\gamma_{11}= & 2 \lambda_{s \phi}-\frac{3}{2}\left(y_{d}^{\alpha}\right)^{2}+\frac{9}{2}\left(y_{u}^{\alpha}\right)^{2} \\
& -\frac{17}{12} g_{1}^{2}-\frac{9}{4} g_{2}^{2}-8 g_{3}^{2}+\gamma_{\phi}^{(Y)}, \\
\gamma_{22}= & 2 \lambda_{s \phi}-\frac{3}{2}\left(y_{u}^{\alpha}\right)^{2}+\frac{9}{2}\left(y_{d}^{\alpha}\right)^{2} \\
& -\frac{5}{12} g_{1}^{2}-\frac{9}{4} g_{2}^{2}-8 g_{3}^{2}+\gamma_{\phi}^{(Y)}, \\
\gamma_{33}= & 2 \lambda_{s \phi}+\frac{9}{2}\left(y_{e}^{\alpha}\right)^{2}-\frac{15}{4} g_{1}^{2}-\frac{9}{4} g_{2}^{2}+\gamma_{\phi}^{(Y)} .
\end{aligned}
$$

$$
\begin{aligned}
& \beta_{C_{u}}=y^{u \dagger} y^{u} C_{u}+2 C_{u} y^{u \dagger} y^{u} \\
& +2 \lambda_{s \phi} C_{u}+\left(y^{u}\right)^{-1} y^{d} C_{d} y^{d \dagger} y^{u} \\
& +\frac{17}{3} g_{1}^{4} C_{B}+9 g_{2}^{4} C_{W}+32 g_{3}^{4} C_{G}-2 \gamma^{\prime}, \\
& \beta_{C_{d}}=y^{d \dagger} y^{d} C_{d}+2 C_{d} y^{d \dagger} y^{d} \\
& +2 \lambda_{s \phi} C_{d}+\left(y^{d}\right)^{-1} y^{u} C_{u} y^{u \dagger} y^{d} \\
& +\frac{5}{3} g_{1}^{4} C_{B}+9 g_{2}^{4} C_{W}+32 g_{3}^{4} C_{G}+2 \gamma^{\prime}, \\
& \beta_{C_{e}}=y^{e \dagger} y^{e} C_{e}+2 C_{e} y^{e^{\dagger}} y^{e} \\
& +2 \lambda_{s \phi} C_{e}+15 g_{1}^{4} C_{B}+9 g_{2}^{4} C_{W}+2 \gamma^{\prime},
\end{aligned}
$$

with 
$\gamma^{\prime} \equiv \operatorname{Tr}\left[y^{e} C_{e} y^{e \dagger}+3 y^{d} C_{d} y^{d \dagger}-3 y^{u} C_{u} y^{u \dagger}\right]$.

$C_{B}, C_{W}$ and $C_{G}$ are the Wilson coefficients of $g_{1}^{2} \mathcal{O}_{s \widetilde{B}}$, $g_{2}^{2} \mathcal{O}_{s \widetilde{W}}$ and $g_{3}^{2} \mathcal{O}_{s \widetilde{G}}$, respectively.

The dependence of the RGEs above on $C_{B}, C_{W}$ and $C_{G}$ was computed already in Ref. [24]. We find exact agreement. Likewise, in Ref. [59] it was shown that $C_{B}, C_{W}$ and $C_{G}$ are scale invariant, i.e. they do not depend on $\mu$. This is consistent with our results, for which all the running of $a_{s} \widetilde{G}, s \widetilde{W}, s \widetilde{B}$ can be accounted for by the running of the corresponding gauge couplings, which are determined by the wave function renormalization of the gauge fields in the background field method.

Finally, the RGEs for the dimension-four operators in the ALP EFT Lagrangian must be provided in order to fully determine the theory. We have obtained these with the help of Pyr@te [72]; they are reported in Appendix C. Let us remind once more that the RGEs of renormalizable interactions are not perturbed by higher-dimensional operators at order $\mathcal{O}(1 / \Lambda)$.

\section{Matching and running below the electroweak scale}

At energies smaller than the EW scale, set by the Higgs vacuum expectation value (VEV), $v \sim 246 \mathrm{GeV}$, the ALP phenomenology must be described by a different EFT, that we call ALP LEFT, organised in inverse powers of $v$, in which the now massive top quark and the Higgs, $Z$ and $W$ bosons are not present. Assuming still $\mathrm{CP}$ conservation, the corresponding ALP LEFT Lagrangian, to dimension five, takes the following form:

$$
\begin{aligned}
\mathcal{L}_{\mathrm{LEFT}}= & \frac{1}{2}\left(\partial_{\mu} s\right)\left(\partial^{\mu} s\right)-\frac{1}{2} \tilde{m}^{2} s^{2}-\frac{\tilde{\lambda}_{s}}{4 !} s^{4} \\
& -\frac{1}{4} A_{\mu \nu} A^{\mu \nu}-\frac{1}{4} G_{\mu \nu}^{A} G^{A \mu \nu} \\
& +\sum_{\psi=u, d, e}\left\{\overline{\psi^{\alpha}} \mathrm{i} \not D \psi^{\alpha}-\left[\left(\tilde{m}_{\psi}\right)_{\alpha \beta} \overline{\psi_{L}^{\alpha}} \psi_{R}^{\beta}\right.\right. \\
& \left.\left.-s \mathrm{i}\left(\tilde{c}_{\psi}\right)_{\alpha \beta} \overline{\psi_{L}^{\alpha}} \psi_{R}^{\beta}+\text { h.c. }\right]\right\} \\
& +\tilde{a}_{s \widetilde{G}} s G_{\mu \nu}^{A} \widetilde{G}^{A \mu \nu}+\tilde{a}_{s} \tilde{A}^{s} A_{\mu \nu} \widetilde{A}^{\mu \nu} \\
& +\sum_{\psi=u, d, e}\left\{\left(\tilde{a}_{\psi A}\right)_{\alpha \beta} \overline{\psi_{L}^{\alpha}} \sigma^{\mu \nu} \psi_{R}^{\beta} A_{\mu \nu}\right. \\
& \left.+\left(\tilde{a}_{\psi G}\right)_{\alpha \beta} \overline{\psi_{L}^{\alpha}} \sigma^{\mu \nu} T_{A} \psi_{R}^{\beta} G_{\mu \nu}^{A}+s^{2}\left(\tilde{a}_{\psi}\right)_{\alpha \beta} \overline{\psi_{L}^{\alpha}} \psi_{R}^{\beta}+\text { h.c. }\right\},
\end{aligned}
$$

where $\alpha, \beta$ are flavour indices that run over the three families for $d$ and $e$ and over the lighter two for the case of $u$.

The assumed CP invariance forces all coefficients to be real (matrices in case flavour is involved). When no chiralities for the fermions are explicitly written we assume
$\psi=\psi_{L}+\psi_{R}$ and the covariant derivative in this regime reads $D_{\mu}=\partial_{\mu}-\mathrm{i} \tilde{e} Q A_{\mu}-\mathrm{i} \tilde{g}_{3} T^{A} G_{\mu}^{A}$, with $Q$ being the electric charge. We emphasise that contrary to the ALP EFT above the EW scale, in this case there are (lepton-number conserving) effective operators of the same dimension with and without the ALP. Note that, as explicitly written above, we work in a flavour basis in which mass matrices are not necessarily diagonal. At the level of computation, this is equivalent to promoting the masses to Yukawa couplings of a spurion scalar field which is later set to its VEV. Technically, every time we have to integrate out one of the SM fermions (the top in this case, lighter fermions as we go to lower energies, see below) we go to the physical basis in which the mass matrix is diagonalised; off-diagonal mass terms being generated by running to lower energies.

The following redundant operators arise at dimension five:

$$
\begin{aligned}
\mathcal{L}_{R}= & \sum_{\psi=u, d, e}\left[\left(\tilde{r}_{\psi \square}\right)_{\alpha \beta} \overline{\psi_{L}^{\alpha}} D^{2} \psi_{R}^{\beta}+\mathrm{i}\left(\tilde{r}_{s \psi_{L}}\right)_{\alpha \beta} s \overline{\psi_{L}^{\alpha}} \mathrm{i} \not D \psi_{L}^{\beta}\right. \\
& \left.+\mathrm{i}\left(\tilde{r}_{s \psi_{R}}\right)_{\alpha \beta} s \overline{\psi_{R}^{\alpha}} \mathrm{i} \not D \psi_{R}^{\beta}+\text { h.c. }\right],
\end{aligned}
$$

where again the operators are $\mathrm{CP}$-even for real Wilson coefficients. The purely SMEFT redundant operator can be removed by making use of the relation

$$
D^{2}=\not D^{2}+\frac{\sigma_{\mu \nu}}{2}\left(\tilde{e} Q A^{\mu \nu}+\tilde{g}_{3} G_{A}^{\mu \nu} T_{A}\right) .
$$

The first term in the equation above can then be further reduced by applying the equations of motion for fermions in the ALP LEFT,

$$
\begin{aligned}
\mathrm{i} \not \supset \psi_{\alpha}= & \left(\tilde{m}_{\psi}\right)_{\alpha \beta} \psi_{R}^{\beta}+\left(\tilde{m}_{\psi}^{\dagger}\right)_{\alpha \beta} \psi_{L}^{\beta} \\
& -\mathrm{i}\left(\tilde{c}_{\psi}\right)_{\alpha \beta} s \psi_{R}^{\beta}+\mathrm{i}\left(\tilde{c}_{\psi}^{\dagger}\right)_{\alpha \beta} s \psi_{L}^{\beta} .
\end{aligned}
$$

There is an apparent ambiguity in this process for the $\not D^{2}$ term due to the possibility of performing integration by parts before applying the equations of motion. However, this ambiguity simply corresponds to a chiral rotation and therefore has no physical consequences (see Ref. [73] for a related discussion). ${ }^{4}$ We choose to split the covariant derivative sym-

\footnotetext{
${ }^{4}$ As an example of the mentioned apparent ambiguity, we could choose to apply the equations of motion without the splitting in Eq. (70). In that case we obtain the same contribution to the dimension-five operators but a different contribution to the renormalizable ones. This difference is however removed, after canonical normalization, by the following chiral unitary rotation:
}

$$
\begin{aligned}
& \psi_{L} \rightarrow\left(1+\frac{\tilde{r}_{\psi \square} \tilde{m}_{\psi}^{\dagger}-\tilde{m}_{\psi} \tilde{r}_{\psi \square}^{\dagger}}{4}\right) \psi_{L}, \\
& \psi_{R} \rightarrow\left(1+\frac{\tilde{m}_{\psi}^{\dagger} \tilde{r}_{\psi \square}-\tilde{r}_{\psi \square}^{\dagger} \tilde{m}_{\psi}}{4}\right) \psi_{R} .
\end{aligned}
$$


metrically:

$\not D^{2}=\frac{1}{2}\left(\not D^{2}+\overleftarrow{D D}^{2}\right)$

In this case we obtain the following on-shell equivalence relations (as usual we write our equations in matrix form in flavour space):

$$
\begin{aligned}
& \overline{\psi_{L}} \tilde{r}_{\psi \square} D^{2} \psi_{R}+\text { h.c. }
\end{aligned}
$$

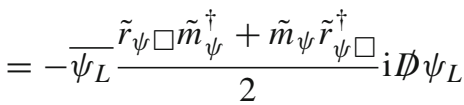

$$
\begin{aligned}
& -\bar{\psi}_{R} \frac{\tilde{r}_{\psi \square}^{\dagger} \tilde{m}_{\psi}+\tilde{m}_{\psi}^{\dagger} \tilde{r}_{\psi \square}}{2} \mathrm{i} \not D \psi_{R} \\
& +\left[\mathrm{i} s \overline{\psi_{L}} \frac{\tilde{m}_{\psi} \tilde{r}_{\psi \square}^{\dagger} \tilde{c}_{\psi}+\tilde{c}_{\psi} \tilde{r}_{\psi \square}^{\dagger} \tilde{m}_{\psi}}{2} \psi_{R}\right. \\
& +s^{2} \overline{\psi_{L}} \tilde{c}_{\psi} \tilde{r}_{\psi \square}^{\dagger} \tilde{c}_{\psi} \psi_{R}+\overline{\psi_{L}} \frac{e Q_{\psi} \tilde{r}_{\psi \square}}{2} \sigma_{\mu \nu} \psi_{R} A^{\mu \nu} \\
& \left.+\overline{\psi_{L}} \frac{\tilde{g}_{3} \tilde{r}_{\psi \square}}{2} T_{A} \sigma_{\mu \nu} \psi_{R} G_{A}^{\mu \nu}+\text { h.c. }\right], \\
& \text { is } \overline{\psi_{L}} \tilde{r}_{s} \psi_{L} \mathrm{i} \not D \psi_{L}+\text { h.c. } \\
& =\mathrm{is} \overline{\psi_{L}} \tilde{r}_{s} \psi_{L} \tilde{m}_{\psi} \psi_{R}+s^{2} \overline{\psi_{L}} \tilde{r}_{s} \psi_{L} \tilde{c}_{\psi} \psi_{R}+\text { h.c. } \\
& \text { is } \overline{\psi_{R}} \tilde{r}_{s \psi_{R}} \mathrm{i} \not D \psi_{R}+\text { h.c. } \\
& =-\mathrm{i} s \overline{\psi_{L}} \tilde{m}_{\psi} \tilde{r}_{s \psi_{R}}^{\dagger} \psi_{R}-s^{2} \overline{\psi_{L}} \tilde{c}_{\psi} \tilde{r}_{s \psi_{R}}^{\dagger} \psi_{R}+\text { h.c.. }
\end{aligned}
$$

The parameters of the ALP LEFT can be fully fixed at the scale $\mu=v$ by requiring that it describes exactly the same physics as the EFT before EWSB at the scale $\mu$. Proceeding this way at tree level, we obtain the following matching conditions for the interactions in Eq. (65):

$$
\begin{array}{ll}
\tilde{e}=g_{2} s_{w}=g_{1} c_{w}, & \tilde{m}^{2}=m^{2}+\frac{\lambda_{s \phi}}{2} v^{2}, \\
\tilde{g}_{3}=g_{3}, & \tilde{\lambda}_{s}=\lambda_{s}-3 \frac{v^{2}}{m_{h}^{2}} \lambda_{s \phi}^{2}, \\
\left(\tilde{m}_{u}\right)_{\alpha \beta}=\frac{v}{\sqrt{2}}\left(y^{u}\right)_{\alpha \beta}, & \left(\tilde{c}_{u}\right)_{\alpha \beta}=\frac{v}{\sqrt{2}}\left(a_{s u \phi}\right)_{\alpha \beta}, \\
\left(\tilde{m}_{d}\right)_{\alpha \beta}=\frac{v}{\sqrt{2}}\left(y^{d}\right)_{\alpha \beta}, & \left(\tilde{c}_{d}\right)_{\alpha \beta}=\frac{v}{\sqrt{2}}\left(a_{s d \phi}\right)_{\alpha \beta}, \\
\left(\tilde{m}_{e}\right)_{\alpha \beta}=\frac{v}{\sqrt{2}}\left(y^{e}\right)_{\alpha \beta}, & \left(\tilde{c}_{e}\right)_{\alpha \beta}=\frac{v}{\sqrt{2}}\left(a_{s e \phi}\right)_{\alpha \beta}, \\
\tilde{a}_{s \widetilde{G}}=a_{s \widetilde{G}}, & \tilde{a}_{s \tilde{A}}=a_{s} \tilde{W}_{w}^{2}+a_{s \widetilde{B}} c_{w}^{2} ;
\end{array}
$$

where, as before, $\alpha$ and $\beta$ are flavour indices that run over the three families for $d$ and $e$ and over the first two for $u ; c_{\omega}$ and $s_{\omega}$ are the cosine and sine of the Weinberg angle, respectively. All the other Wilson coefficients vanish at the order we are computing. The fact that the three coefficients $\tilde{a}_{u, d, e}$ vanish might be surprising at first glance, as the Higgs couples to both $s^{2}$ and to fermionic currents with overall strength $\sim \lambda_{s \phi} y^{\psi} / v$. However, precisely because the Higgs boson sets the scale of light masses [74], i.e. because $y^{\psi} \sim m_{\psi} / v$, the product $\lambda_{s \phi} y^{\psi} / v \sim \lambda_{s \phi} m_{\psi} / v^{2}$ is of higher order in the low-energy power counting and therefore negligible. This is no longer true at dimension six (it is in the pure SM EFT even at dimension six because an s-channel Higgs always involves two powers of Yukawa couplings). Similarly, a possible contribution proportional to two powers of $v a_{s u \phi}$ is also higher order in the $1 / \Lambda$ expansion.

At energies below the bottom quark mass $m_{b}$, the effective Lagrangian takes exactly the same form as in Eq. (65) except that the flavour indices now run only over the remaining fermions and the Wilson coefficients in the new EFT have to be matched accordingly. The same logic applies as we cross new fermionic thresholds. At the order we are considering, however, the matching is straightforward and the only thing we have to do is to remove the Wilson coefficients involving the particle being integrated out. The only exception arises if $\tilde{c}_{\psi}$ is unsuppressed, in which case integrating out a massive fermion would result in the following matching condition:

$\left(\tilde{a}_{\psi}\right)_{\alpha \beta}=-\frac{\left(\tilde{c}_{\psi}\right)_{\alpha \gamma}\left(\tilde{c}_{\psi}\right)_{\gamma \beta}}{\left(\tilde{m}_{\psi}\right)_{\gamma}}($ no sum over $\gamma)$,

where $\gamma$ corresponds to the flavour that is being integrated out while $\alpha$ and $\beta$ run over lighter flavours of the same type of fermion. This term is higher order if the ALP LEFT is obtained from the ALP EFT. However, we prefer to keep this section completely general, independently of which theory completes the ALP LEFT in the UV.

The running of the Wilson coefficients between different thresholds is very different from the running above the EW scale (the operators in Table 1). In particular, operators of different energy dimensions, as well as operators with and without the ALP field, will now mix under renormalization.

\subsection{Divergences at one loop}

Similar to how we proceeded in Sect. 3, we fix the ALP LEFT divergences by computing a reduced set of 1PI amplitudes, the $1 / v$ term of which we reproduce below. Since the dimension-five operators mix into renormalizable ones, we start with the divergences that can be absorbed in the renormalizable operators. In particular, the divergences associated to the kinetic terms can be parametrised, at the one-loop order, in terms of the wave function renormalization factors as follows:

$$
\begin{aligned}
\mathcal{L}_{\mathrm{kin}}= & \overline{\psi_{L}}\left(1-\delta Z_{L}\right) \mathrm{i} \not D \psi_{L}+\overline{\psi_{R}}\left(1-\delta Z_{R}\right) \mathrm{i} \not D \psi_{R} \\
& +\frac{1}{2}\left(1-\delta Z_{S}\right)\left(\partial_{\mu} s\right)\left(\partial^{\mu} s\right)-\frac{1}{4}\left(1-\delta Z_{A}\right) A_{\mu \nu} A^{\mu \nu} \\
& -\frac{1}{4}\left(1-\delta Z_{G}\right) G_{\mu \nu}^{A} G^{A \mu \nu}
\end{aligned}
$$


where the wave function renormalization factors are defined in general by

$Z=1+\delta Z$,

and the relative minus sign is due to the fact that the $Z$ factors are conventionally defined to absorb, rather than parametrise, the corresponding divergences. As discussed above, the wave function renormalization factors have contributions proportional to only renormalizable couplings (that contribute to the running of the non-renormalizable ones; see Figs. 12, 13, 14, 15,16 and 17) and to dimension-five couplings (that contribute to the mixing into renormalizable ones). We obtain the following result:

$$
\begin{aligned}
& Z_{e_{L}}= 1-\frac{\alpha}{4 \pi \epsilon}-\frac{1}{32 \pi^{2} \epsilon}\left(\tilde{c}_{e} \tilde{c}_{e}^{\dagger}\right) \\
&-\frac{3 \tilde{e}}{16 \pi^{2} \epsilon}\left(\tilde{m}_{e} \tilde{a}_{e A}^{\dagger}+\tilde{a}_{e A} \tilde{m}_{e}^{\dagger}\right), \\
& Z_{e_{R}}= 1-\frac{\alpha}{4 \pi \epsilon}-\frac{1}{32 \pi^{2} \epsilon}\left(\tilde{c}_{e}^{\dagger} \tilde{c}_{e}\right) \\
&-\frac{3 \tilde{e}}{16 \pi^{2} \epsilon}\left(\tilde{a}_{e A}^{\dagger} \tilde{m}_{e}+\tilde{m}_{e}^{\dagger} \tilde{a}_{e A}\right), \\
& Z_{d_{L}}= 1-\frac{1}{3 \pi \epsilon}\left[\frac{1}{12} \alpha+\alpha_{s}\right]-\frac{1}{32 \pi^{2} \epsilon}\left(\tilde{c}_{d} \tilde{c}_{d}^{\dagger}\right) \\
&-\frac{\tilde{e}}{16 \pi^{2} \epsilon}\left(\tilde{m}_{d} \tilde{a}_{d A}^{\dagger}+\tilde{a}_{d A} \tilde{m}_{d}^{\dagger}\right) \\
&+\frac{\tilde{g}_{3}}{4 \pi^{2} \epsilon}\left(\tilde{m}_{d} \tilde{a}_{d G}^{\dagger}+\tilde{a}_{d G} \tilde{m}_{d}^{\dagger}\right), \\
& Z_{d_{R}}= 1-\frac{1}{3 \pi \epsilon}\left[\frac{1}{12} \alpha+\alpha_{s}\right]-\frac{1}{32 \pi^{2} \epsilon}\left(\tilde{c}_{d}^{\dagger} \tilde{c}_{d}\right) \\
&-\frac{\tilde{e}}{16 \pi^{2} \epsilon}\left(\tilde{a}_{d A}^{\dagger} \tilde{m}_{d}+\tilde{m}_{d}^{\dagger} \tilde{a}_{d A}\right) \\
&+\frac{\tilde{g}_{3}}{4 \pi^{2} \epsilon}\left(\tilde{a}_{d G}^{\dagger} \tilde{m}_{d}+\tilde{m}_{d}^{\dagger} \tilde{a}_{d G}\right), \\
& Z_{A}= 1-\frac{1}{3 \pi \epsilon}\left[\frac{1}{3} \alpha+\alpha_{s}\right]-\frac{1}{32 \pi^{2} \epsilon}\left(\tilde{c}_{u} \tilde{c}_{u}^{\dagger}\right) \\
&+\frac{\tilde{e}}{2 \pi \pi^{2} \epsilon} \operatorname{Tr}\left[\left(\tilde{a}_{e A}^{\dagger} \tilde{m}_{e}+\tilde{m}_{e}^{\dagger} \tilde{a}_{e A}\right)\right. \\
& Z_{u_{R}}= 1-\frac{1}{3 \pi \epsilon}\left[\frac{1}{3} \alpha+\alpha_{s}\right]-\frac{1}{32 \pi^{2} \epsilon}\left(\tilde{c}_{u}^{\dagger} \tilde{c}_{u}\right) \\
&+\frac{2 \tilde{e}}{16 \pi^{2} \epsilon}\left(\tilde{a}_{u A}^{\dagger} \tilde{m}_{u}+\tilde{m}_{u}^{\dagger} \tilde{a}_{u A}\right) \\
&+\frac{2 \tilde{e}}{16 \pi^{2} \epsilon}\left(\tilde{m}_{u} \tilde{a}_{u A}^{\dagger}+\tilde{a}_{u A} \tilde{m}_{u}^{\dagger}\right) \\
&+\frac{\tilde{g}_{3}}{4 \pi^{2} \epsilon}\left(\tilde{m}_{u} \tilde{a}_{u G}^{\dagger}+\tilde{a}_{u G}+\tilde{m}_{u}^{\dagger} \tilde{a}_{u G}\right), \\
&\left.\tilde{m}_{u}^{\dagger}\right), \\
&\left.\tilde{a}^{2} n_{d}+\frac{4}{3} n_{u}\right] \\
&=
\end{aligned}
$$

$$
\begin{aligned}
& \left.-2\left(\tilde{a}_{u A}^{\dagger} \tilde{m}_{u}+\tilde{m}_{u}^{\dagger} \tilde{a}_{u A}\right)+\left(\tilde{a}_{d A}^{\dagger} \tilde{m}_{d}+\tilde{m}_{d}^{\dagger} \tilde{a}_{d A}\right)\right], \\
Z_{G}= & 1+\frac{\alpha_{s}}{4 \pi \epsilon}\left[11-\frac{2}{3}\left(n_{u}+n_{d}\right)\right] \\
& -\frac{\tilde{g}_{3}}{4 \pi^{2} \epsilon} \operatorname{Tr}\left[\tilde{a}_{d G}^{\dagger} \tilde{m}_{d}+\tilde{m}_{d}^{\dagger} \tilde{a}_{d G}+\tilde{a}_{u G}^{\dagger} \tilde{m}_{u}+\tilde{m}_{u}^{\dagger} \tilde{a}_{u G}\right], \\
Z_{s}= & 1-\frac{1}{8 \pi^{2} \epsilon} \operatorname{Tr}\left[\tilde{c}_{e} \tilde{c}_{e}^{\dagger}+3\left(\tilde{c}_{d} \tilde{c}_{d}^{\dagger}+\tilde{c}_{u} \tilde{c}_{u}^{\dagger}\right)\right],
\end{aligned}
$$

where we have defined $\alpha=\tilde{e}^{2} /(4 \pi)$ and $\alpha_{s}={\tilde{g_{3}}}^{2} /(4 \pi)$. For the remaining couplings we only need to consider diagrams with a single insertion of a dimension-five operator. The result is given below, organised according to the amplitudes we have used to compute the corresponding divergences. We provide all the relevant diagrams in Appendix D.

- $s\left(p_{1}\right) \rightarrow s\left(p_{2}\right)$

The amplitude given by the diagrams in Fig. 14 fixes the divergence of the mass term,

$$
\begin{aligned}
\tilde{m}^{\prime 2}= & -\frac{3}{4 \pi^{2} \epsilon}\left(\operatorname{Tr}\left[\tilde{m}_{d}^{\dagger} \tilde{a}_{d} \tilde{m}_{d}^{\dagger} \tilde{m}_{d}+\tilde{m}_{d}^{\dagger} \tilde{m}_{d} \tilde{a}_{d}^{\dagger} \tilde{m}_{d}\right]\right. \\
& \left.+\operatorname{Tr}\left[\tilde{m}_{u}^{\dagger} \tilde{a}_{u} \tilde{m}_{u}^{\dagger} \tilde{m}_{u}+\tilde{m}_{u}^{\dagger} \tilde{m}_{u} \tilde{a}_{u}^{\dagger} \tilde{m}_{u}\right]\right) \\
& -\frac{1}{4 \pi^{2} \epsilon} \operatorname{Tr}\left[\tilde{m}_{e}^{\dagger} \tilde{a}_{e} \tilde{m}_{e}^{\dagger} \tilde{m}_{e}+\tilde{m}_{e}^{\dagger} \tilde{m}_{e} \tilde{a}_{e}^{\dagger} \tilde{m}_{e}\right] .
\end{aligned}
$$

- $\psi\left(p_{1}\right) \rightarrow \psi\left(p_{2}\right)$

The diagrams for $\psi=e, u, d$ are shown in Figs. 15, 16 and 17 . They contribute to the fermion mass divergences,

$$
\begin{aligned}
\tilde{m}_{e}^{\prime}= & -\frac{3}{8 \pi^{2} \epsilon} \tilde{e}\left(\tilde{m}_{e} \tilde{m}_{e}^{\dagger} \tilde{a}_{e A}+\tilde{a}_{e A} \tilde{m}_{e}^{\dagger} \tilde{m}_{e}\right)+\frac{1}{16 \pi^{2} \epsilon} \tilde{m}^{2} \tilde{a}_{e}, \\
\tilde{m}_{u}^{\prime}= & \frac{2}{8 \pi^{2} \epsilon} \tilde{e}\left(\tilde{m}_{u} \tilde{m}_{u}^{\dagger} \tilde{a}_{u A}+\tilde{a}_{u A} \tilde{m}_{u}^{\dagger} \tilde{m}_{u}\right) \\
& +\frac{1}{2 \pi^{2} \epsilon} \tilde{g}_{3}\left(\tilde{m}_{u} \tilde{m}_{u}^{\dagger} \tilde{a}_{u G}+\tilde{a}_{u G} \tilde{m}_{u}^{\dagger} \tilde{m}_{u}\right)+\frac{1}{16 \pi^{2} \epsilon} \tilde{m}^{2} \tilde{a}_{u}, \\
\tilde{m}_{d}^{\prime}= & -\frac{1}{8 \pi^{2} \epsilon} \tilde{e}\left(\tilde{m}_{d} \tilde{m}_{d}^{\dagger} \tilde{a}_{d A}+\tilde{a}_{d A} \tilde{m}_{d}^{\dagger} \tilde{m}_{d}\right) \\
& +\frac{1}{2 \pi^{2} \epsilon} \tilde{g}_{3}\left(\tilde{m}_{d} \tilde{m}_{d}^{\dagger} \tilde{a}_{d G}+\tilde{a}_{d G} \tilde{m}_{d}^{\dagger} \tilde{m}_{d}\right)+\frac{1}{16 \pi^{2} \epsilon} \tilde{m}^{2} \tilde{a}_{d},
\end{aligned}
$$

as well as to the dimension-five contribution to the kinetic terms reported above.

- $s\left(p_{1}\right) s\left(p_{2}\right) \rightarrow s\left(p_{3}\right) s\left(p_{4}\right)$

The corresponding amplitude, represented by the diagrams in Fig. 18, fixes the ALP quartic coupling,

$\tilde{\lambda}_{s}^{\prime}=-\frac{3}{\pi^{2} \epsilon}\left[\operatorname{Tr}_{e}^{\tilde{\lambda}}+3\left(\operatorname{Tr}_{u}^{\tilde{\lambda}}+\operatorname{Tr}_{d}^{\tilde{\lambda}}\right)\right]$ 
where we have defined

$$
\begin{aligned}
\operatorname{Tr}_{\psi}^{\tilde{\lambda}} \equiv & \operatorname{Tr}\left[\tilde{a}_{\psi} \tilde{c}_{\psi}^{\dagger} \tilde{c}_{\psi} \tilde{m}_{\psi}^{\dagger}+\tilde{a}_{\psi} \tilde{m}_{\psi}^{\dagger} \tilde{c}_{\psi} \tilde{c}_{\psi}^{\dagger}-\tilde{a}_{\psi}^{\dagger} \tilde{c}_{\psi} \tilde{m}_{\psi}^{\dagger} \tilde{c}_{\psi}\right. \\
& \left.-\tilde{a}_{\psi} \tilde{c}_{\psi}^{\dagger} \tilde{m}_{\psi} \tilde{c}_{\psi}^{\dagger}+\tilde{a}_{\psi}^{\dagger} \tilde{c}_{\psi} \tilde{c}_{\psi}^{\dagger} \tilde{m}_{\psi}+\tilde{a}_{\psi}^{\dagger} \tilde{m}_{\psi} \tilde{c}_{\psi}^{\dagger} \tilde{c}_{\psi}\right]
\end{aligned}
$$

- $s\left(p_{1}\right) \rightarrow \overline{\psi^{\alpha}}\left(p_{2}\right) \psi^{\beta}\left(p_{3}\right)$

The corresponding diagrams are shown in Figs. 19, 20 and 21 for $\psi=e, u, d$. We obtain the following divergences for the renormalizable operators:

$$
\begin{aligned}
\tilde{c}_{e}^{\prime}= & \frac{1}{8 \pi^{2} \epsilon}\left(\tilde{a}_{e} \tilde{m}_{e}^{\dagger} \tilde{c}_{e}+\tilde{c}_{e} \tilde{m}_{e}^{\dagger} \tilde{a}_{e}\right) \\
& +\frac{3 \tilde{e}}{8 \pi^{2} \epsilon}\left(\tilde{m}_{e} \tilde{c}_{e}^{\dagger} \tilde{a}_{e A}+\tilde{a}_{e A} \tilde{c}_{e}^{\dagger} \tilde{m}_{e}-\tilde{c}_{e} \tilde{m}_{e}^{\dagger} \tilde{a}_{e A}-\tilde{a}_{e A} \tilde{m}_{e}^{\dagger} \tilde{c}_{e}\right),
\end{aligned}
$$

$$
\begin{aligned}
\tilde{c}_{u}^{\prime}= & \frac{1}{8 \pi^{2} \epsilon}\left(\tilde{a}_{u} \tilde{m}_{u}^{\dagger} \tilde{c}_{u}+\tilde{c}_{u} \tilde{m}_{u}^{\dagger} \tilde{a}_{u}\right) \\
& -\frac{2 \tilde{e}}{8 \pi^{2} \epsilon}\left(\tilde{m}_{u} \tilde{c}_{u}^{\dagger} \tilde{u}_{u A}+\tilde{a}_{u A} \tilde{c}_{u}^{\dagger} \tilde{m}_{u}-\tilde{c}_{u} \tilde{m}_{u}^{\dagger} \tilde{a}_{u A}-\tilde{a}_{u A} \tilde{m}_{u}^{\dagger} \tilde{c}_{u}\right) \\
& -\frac{\tilde{g}_{3}}{2 \pi^{2} \epsilon}\left(\tilde{m}_{u} \tilde{c}_{u}^{\dagger} \tilde{a}_{u G}+\tilde{a}_{u G} \tilde{c}_{u}^{\dagger} \tilde{m}_{u}-\tilde{c}_{u} \tilde{m}_{u}^{\dagger} \tilde{a}_{u G}-\tilde{a}_{u G} \tilde{m}_{u}^{\dagger} \tilde{c}_{u}\right),
\end{aligned}
$$

$$
\begin{aligned}
\tilde{c}_{d}^{\prime}= & \frac{1}{8 \pi^{2} \epsilon}\left(\tilde{a}_{d} \tilde{m}_{d}^{\dagger} \tilde{c}_{d}+\tilde{c}_{d} \tilde{m}_{d}^{\dagger} \tilde{a}_{d}\right) \\
& +\frac{\tilde{e}}{8 \pi^{2} \epsilon}\left(\tilde{m}_{d} \tilde{c}_{d}^{\dagger} \tilde{a}_{d A}+\tilde{a}_{d A} \tilde{c}_{d}^{\dagger} \tilde{m}_{d}-\tilde{c}_{d} \tilde{m}_{d}^{\dagger} \tilde{a}_{d A}-\tilde{a}_{d A} \tilde{m}_{d}^{\dagger} \tilde{c}_{d}\right) \\
& -\frac{\tilde{g}_{3}}{2 \pi^{2} \epsilon}\left(\tilde{m}_{d} \tilde{c}_{d}^{\dagger} \tilde{a}_{d G}+\tilde{a}_{d G} \tilde{c}_{d}^{\dagger} \tilde{m}_{d}-\tilde{c}_{d} \tilde{m}_{d}^{\dagger} \tilde{a}_{d G}-\tilde{a}_{d G} \tilde{m}_{d}^{\dagger} \tilde{c}_{d}\right),
\end{aligned}
$$

and for the non-renormalizable ones:

$$
\begin{aligned}
\tilde{r}_{s e_{L}}^{\prime}= & -\frac{1}{16 \pi^{2} \epsilon} \tilde{a}_{e} \tilde{c}_{e}^{\dagger}+\frac{3}{2 \pi \epsilon} \alpha \tilde{a}_{s \widetilde{A}}-\frac{3 \tilde{e}}{16 \pi^{2} \epsilon} \tilde{c}_{e} \tilde{a}_{e A}^{\dagger} \\
\tilde{r}_{s e_{R}}^{\prime}= & \frac{1}{16 \pi^{2} \epsilon} \tilde{a}_{e}^{\dagger} \tilde{c}_{e}-\frac{3}{2 \pi \epsilon} \alpha \tilde{a}_{s} \tilde{A}+\frac{3 \tilde{e}}{16 \pi^{2} \epsilon} \tilde{c}_{e}^{\dagger} \tilde{a}_{e A} \\
\tilde{r}_{s u_{L}}^{\prime}= & -\frac{1}{16 \pi^{2} \epsilon} \tilde{a}_{u} \tilde{c}_{u}^{\dagger}+\frac{2}{\pi \epsilon}\left(\frac{1}{3} \alpha \tilde{a}_{s} \tilde{A}+\alpha_{s} \tilde{a}_{s \widetilde{G}}\right) \\
& +\frac{2 \tilde{e}}{16 \pi^{2} \epsilon} \tilde{c}_{u} \tilde{a}_{u A}^{\dagger}+\frac{1}{4 \pi^{2} \epsilon} \tilde{g}_{3} \tilde{c}_{u} \tilde{a}_{u G}^{\dagger} \\
\tilde{r}_{s u_{R}}^{\prime}= & \frac{1}{16 \pi^{2} \epsilon} \tilde{a}_{u}^{\dagger} \tilde{c}_{u}-\frac{2}{\pi \epsilon}\left(\frac{1}{3} \alpha \tilde{a}_{s} \tilde{A}^{+} \alpha_{s} \tilde{a}_{s \widetilde{G}}\right) \\
& -\frac{2 \tilde{e}}{16 \pi^{2} \epsilon} \tilde{c}_{u}^{\dagger} \tilde{a}_{u A}+\frac{1}{4 \pi^{2} \epsilon} \tilde{g}_{3} \tilde{c}_{u}^{\dagger} \tilde{a}_{u G} \\
\tilde{r}_{s d_{L}}^{\prime}= & -\frac{1}{16 \pi^{2} \epsilon} \tilde{a}_{d} \tilde{c}_{d}^{\dagger}+\frac{2}{\pi \epsilon}\left(\frac{1}{12} \alpha \tilde{a}_{s} \tilde{A}+\alpha_{s} \tilde{a}_{s \widetilde{G}}\right) \\
& -\frac{\tilde{e}}{16 \pi^{2} \epsilon} \tilde{c}_{d} \tilde{a}_{d A}^{\dagger}+\frac{1}{4 \pi^{2} \epsilon} \tilde{g}_{3} \tilde{c}_{d} \tilde{a}_{d G}^{\dagger} \\
\tilde{r}_{s d_{R}}^{\prime}= & \frac{1}{16 \pi^{2} \epsilon} \tilde{a}_{d}^{\dagger} \tilde{c}_{d}-\frac{2}{\pi \epsilon}\left(\frac{1}{12} \alpha \tilde{a}_{s} \tilde{A}^{+}+\alpha_{s} \tilde{a}_{s} \tilde{G}\right)
\end{aligned}
$$

$$
+\frac{\tilde{e}}{16 \pi^{2} \epsilon} \tilde{c}_{d}^{\dagger} \tilde{a}_{d A}+\frac{1}{4 \pi^{2} \epsilon} \tilde{g}_{3} \tilde{c}_{d}^{\dagger} \tilde{a}_{d G}
$$

- $A\left(p_{1}\right) \rightarrow \overline{\psi^{\alpha}}\left(p_{2}\right) \psi^{\beta}\left(p_{3}\right)$

The corresponding diagrams are shown in Figs. 22, 23 and 24 for $\psi=e, u, d$, respectively. This process fixes the divergences

$\begin{aligned} \tilde{a}_{e A}^{\prime} & =-\frac{\tilde{e}}{16 \pi^{2} \epsilon}\left(\tilde{e} \tilde{a}_{e A}+2 \tilde{c}_{e} \tilde{a}_{s \tilde{A}}\right), \\ \tilde{a}_{u A}^{\prime} & =-\frac{\tilde{e}}{12 \pi^{2} \epsilon}\left(\frac{1}{3} \tilde{e} \tilde{a}_{u A}-\tilde{c}_{u} \tilde{a}_{s \tilde{A}}\right)-\frac{\tilde{e}}{18 \pi^{2} \epsilon} \tilde{g}_{3} \tilde{a}_{u G},\end{aligned}$

$\tilde{a}_{d A}^{\prime}=-\frac{\tilde{e}}{24 \pi^{2} \epsilon}\left(\frac{1}{6} \tilde{e} \tilde{a}_{d A}+\tilde{c}_{d} \tilde{a}_{s \tilde{A}}\right)+\frac{\tilde{e}}{36 \pi^{2} \epsilon} \tilde{g}_{3} \tilde{a}_{d G}$,

$\tilde{r}_{e \square}^{\prime}=\frac{3}{8 \pi^{2} \epsilon} \tilde{e} \tilde{a}_{e A}$,

$\tilde{r}_{u \square}^{\prime}=-\frac{1}{4 \pi^{2} \epsilon} \tilde{e} \tilde{a}_{u A}-\frac{1}{2 \pi^{2} \epsilon} \tilde{g}_{3} \tilde{a}_{u G}$,

$\tilde{r}_{d \square}^{\prime}=\frac{1}{8 \pi^{2} \epsilon} \tilde{e} \tilde{a}_{d A}-\frac{1}{2 \pi^{2} \epsilon} \tilde{g}_{3} \tilde{a}_{d G}$,

as well as a contribution to the fermion kinetic term, which we have provided fully (i.e. including all contributions up to order $1 / v)$ above.

- $G\left(p_{1}\right) \rightarrow \overline{\psi^{\alpha}}\left(p_{2}\right) \psi^{\beta}\left(p_{3}\right)$

The diagrams for $\psi=u, d$ are respectively shown in Figs. 25 and 26. Similar to the previous case, we obtain:

$\tilde{a}_{u G}^{\prime}=\frac{1}{8 \pi^{2} \epsilon} \tilde{g}_{3} \tilde{c}_{u} \tilde{a}_{s \widetilde{G}}+\frac{7}{6 \pi \epsilon} \alpha_{s} \tilde{a}_{u G}-\frac{1}{24 \pi^{2} \epsilon} \tilde{e} \tilde{g}_{3} \tilde{a}_{u A}$,

$\tilde{a}_{d G}^{\prime}=\frac{1}{8 \pi^{2} \epsilon} \tilde{g}_{3} \tilde{c}_{d} \tilde{a}_{s} \widetilde{G}+\frac{7}{6 \pi \epsilon} \alpha_{s} \tilde{a}_{d G}+\frac{1}{48 \pi^{2} \epsilon} \tilde{e} \tilde{g}_{3} \tilde{a}_{d A}$,

as well as cross-check the previous redundant operators and contribution to the kinetic terms for the quarks.

- $s\left(p_{1}\right) s\left(p_{2}\right) \rightarrow \overline{\psi^{\alpha}}\left(p_{3}\right) \psi^{\beta}\left(p_{4}\right)$

The diagrams for $\psi=u, d, e$ are given in Figs. 27, 28 and 29. We get:

$$
\begin{aligned}
\tilde{a}_{e}^{\prime}= & {\left[\frac{1}{\pi \epsilon} \alpha-\frac{\tilde{\lambda}_{s}}{32 \pi^{2} \epsilon}\right] \tilde{a}_{u} } \\
& +\frac{1}{16 \pi^{2} \epsilon}\left(\tilde{c}_{e} \tilde{a}_{e}^{\dagger} \tilde{c}_{e}-2 \tilde{a}_{e} \tilde{c}_{e}^{\dagger} \tilde{c}_{e}-2 \tilde{c}_{e} \tilde{c}_{e}^{\dagger} \tilde{a}_{e}\right) \\
& +\frac{3 \tilde{e}}{8 \pi^{2} \epsilon}\left(\tilde{c}_{e} \tilde{c}_{e}^{\dagger} \tilde{a}_{e A}+\tilde{a}_{e A} \tilde{c}_{e}^{\dagger} \tilde{c}_{e}\right), \\
\tilde{a}_{u}^{\prime}= & {\left[\frac{4}{3 \pi \epsilon}\left(\frac{1}{3} \alpha+\alpha_{s}\right)-\frac{\tilde{\lambda}_{s}}{32 \pi^{2} \epsilon}\right] \tilde{a}_{u} } \\
& +\frac{1}{16 \pi^{2} \epsilon}\left(\tilde{c}_{u} \tilde{a}_{u}^{\dagger} \tilde{c}_{u}-2 \tilde{a}_{u} \tilde{c}_{u}^{\dagger} \tilde{c}_{u}-2 \tilde{c}_{u} \tilde{c}_{u}^{\dagger} \tilde{a}_{u}\right)
\end{aligned}
$$




$$
\begin{aligned}
& -\frac{\tilde{e}}{4 \pi^{2} \epsilon}\left(\tilde{c}_{u} \tilde{c}_{u}^{\dagger} \tilde{a}_{u A}+\tilde{a}_{u A} \tilde{c}_{u}^{\dagger} \tilde{c}_{u}\right) \\
& -\frac{\tilde{g}_{3}}{2 \pi^{2} \epsilon}\left(\tilde{c}_{u} \tilde{c}_{u}^{\dagger} \tilde{a}_{u G}+\tilde{a}_{u G} \tilde{c}_{u}^{\dagger} \tilde{c}_{u}\right), \\
\tilde{a}_{d}^{\prime}= & {\left[\frac{1}{3 \pi \epsilon}\left(\frac{1}{3} \alpha+4 \alpha_{s}\right)-\frac{\tilde{\lambda}_{s}}{32 \pi^{2} \epsilon}\right] \tilde{a}_{d} } \\
& +\frac{1}{16 \pi^{2} \epsilon}\left(\tilde{c}_{d} \tilde{a}_{d}^{\dagger} \tilde{c}_{d}-2 \tilde{a}_{d} \tilde{c}_{d}^{\dagger} \tilde{c}_{d}-2 \tilde{c}_{d} \tilde{c}_{d}^{\dagger} \tilde{a}_{d}\right) \\
& +\frac{\tilde{e}}{8 \pi^{2} \epsilon}\left(\tilde{c}_{d} \tilde{c}_{d}^{\dagger} \tilde{a}_{d A}+\tilde{a}_{d A} \tilde{c}_{d}^{\dagger} \tilde{c}_{d}\right) \\
& -\frac{\tilde{g}_{3}}{2 \pi^{2} \epsilon}\left(\tilde{c}_{d} \tilde{c}_{d}^{\dagger} \tilde{a}_{d G}+\tilde{a}_{d G} \tilde{c}_{d}^{\dagger} \tilde{c}_{d}\right) .
\end{aligned}
$$

- $s\left(p_{1}\right) \rightarrow V\left(p_{2}\right) V\left(p_{3}\right)$

The diagrams for $V=A, G$ are given in Figs. 30 and 31 .

The corresponding divergences read:

$$
\begin{aligned}
\tilde{a}_{s \tilde{A}}= & \frac{\tilde{e}}{8 \pi^{2} \epsilon} \operatorname{Tr}\left[-\left(\tilde{c}_{e} \tilde{a}_{e A}^{\dagger}+\tilde{c}_{e}^{\dagger} \tilde{a}_{e A}\right)\right. \\
& \left.-\left(\tilde{c}_{d} \tilde{a}_{d A}^{\dagger}+\tilde{c}_{d}^{\dagger} \tilde{a}_{d A}\right)+2\left(\tilde{c}_{u} \tilde{a}_{u A}^{\dagger}+\tilde{c}_{u}^{\dagger} \tilde{a}_{u A}\right)\right],
\end{aligned}
$$

$$
\tilde{a}_{s \tilde{G}}=\frac{\tilde{g}_{3}}{16 \pi^{2} \epsilon} \operatorname{Tr}\left[\tilde{c}_{d} \tilde{a}_{d G}^{\dagger}+\tilde{c}_{d}^{\dagger} \tilde{a}_{d G}+\tilde{c}_{u} \tilde{a}_{u G}^{\dagger}+\tilde{c}_{u}^{\dagger} \tilde{a}_{u G}\right] .
$$

\subsection{Eliminating redundancy}

We can now go to the on-shell basis by using the redundancy relations in Eqs. (71)-(73). The kinetic terms for fermions receive an extra contribution from the coefficient of the redundant operators,

$$
\begin{aligned}
& -\delta Z_{\psi_{L}} \rightarrow-\delta Z_{\psi_{L}}-\frac{\tilde{r}_{\psi \square} \tilde{m}_{\psi}^{\dagger}+\tilde{m}_{\psi} \tilde{r}_{\psi \square}^{\dagger}}{2}, \\
& -\delta Z_{\psi_{R}} \rightarrow-\delta Z_{\psi_{R}}-\frac{\tilde{r}_{\psi \square}^{\dagger} \tilde{m}_{\psi}+\tilde{m}_{\psi}^{\dagger} \tilde{r}_{\psi \square}}{2} .
\end{aligned}
$$

Upon replacing the values in Eqs. (83)-(88) and Eqs. (109)(111), we find that the contributions of dimension-five operators to the fermion kinetic terms precisely cancel in the on-shell basis. The resulting wave function renormalization factors then read in the on-shell basis:

$$
\begin{aligned}
& Z_{e_{L}}=1-\frac{\alpha}{4 \pi \epsilon}-\frac{1}{32 \pi^{2} \epsilon}\left(\tilde{c}_{e} \tilde{c}_{e}^{\dagger}\right), \\
& Z_{e_{R}}=1-\frac{\alpha}{4 \pi \epsilon}-\frac{1}{32 \pi^{2} \epsilon}\left(\tilde{c}_{e}^{\dagger} \tilde{c}_{e}\right), \\
& Z_{d_{L}}=1-\frac{1}{3 \pi \epsilon}\left[\frac{1}{12} \alpha+\alpha_{s}\right]-\frac{1}{32 \pi^{2} \epsilon}\left(\tilde{c}_{d} \tilde{c}_{d}^{\dagger}\right), \\
& Z_{d_{R}}=1-\frac{1}{3 \pi \epsilon}\left[\frac{1}{12} \alpha+\alpha_{s}\right]-\frac{1}{32 \pi^{2} \epsilon}\left(\tilde{c}_{d}^{\dagger} \tilde{c}_{d}\right),
\end{aligned}
$$

$$
\begin{aligned}
Z_{u_{L}}= & 1-\frac{1}{3 \pi \epsilon}\left[\frac{1}{3} \alpha+\alpha_{s}\right]-\frac{1}{32 \pi^{2} \epsilon}\left(\tilde{c}_{u} \tilde{c}_{u}^{\dagger}\right), \\
Z_{u_{R}}= & 1-\frac{1}{3 \pi \epsilon}\left[\frac{1}{3} \alpha+\alpha_{s}\right]-\frac{1}{32 \pi^{2} \epsilon}\left(\tilde{c}_{u}^{\dagger} \tilde{c}_{u}\right), \\
Z_{A}= & 1-\frac{\alpha}{3 \pi \epsilon}\left[n_{\ell}+\frac{1}{3} n_{d}+\frac{4}{3} n_{u}\right] \\
& -\frac{\tilde{e}}{2 \pi^{2} \epsilon} \operatorname{Tr}\left[-\left(\tilde{a}_{e A}^{\dagger} \tilde{m}_{e}+\tilde{m}_{e}^{\dagger} \tilde{a}_{e A}\right)\right. \\
& \left.+2\left(\tilde{a}_{u A}^{\dagger} \tilde{m}_{u}+\tilde{m}_{u}^{\dagger} \tilde{a}_{u A}\right)-\left(\tilde{a}_{d A}^{\dagger} \tilde{m}_{d}+\tilde{m}_{d}^{\dagger} \tilde{a}_{d A}\right)\right], \\
Z_{G}= & 1+\frac{\alpha_{s}}{4 \pi \epsilon}\left[11-\frac{2}{3}\left(n_{u}+n_{d}\right)\right] \\
& -\frac{\tilde{g}_{3}}{4 \pi^{2} \epsilon} \operatorname{Tr}\left[\tilde{a}_{d G}^{\dagger} \tilde{m}_{d}+\tilde{m}_{d}^{\dagger} \tilde{a}_{d G}+\tilde{a}_{u G}^{\dagger} \tilde{m}_{u}+\tilde{m}_{u}^{\dagger} \tilde{a}_{u G}\right], \\
Z_{s}= & 1-\frac{1}{8 \pi^{2} \epsilon} \operatorname{Tr}\left[\tilde{c}_{e} \tilde{c}_{e}^{\dagger}+3\left(\tilde{c}_{d} \tilde{c}_{d}^{\dagger}+\tilde{c}_{u} \tilde{c}_{u}^{\dagger}\right)\right] .
\end{aligned}
$$

We also have a contribution to renormalizable coefficients from redundant ones:

$$
\begin{aligned}
& \tilde{c}_{\psi} \rightarrow \tilde{c}_{\psi}+\frac{\tilde{m}_{\psi} \tilde{r}_{\psi \square}^{\dagger} \tilde{c}_{\psi}+\tilde{c}_{\psi} \tilde{r}_{\psi \square}^{\dagger} \tilde{m}_{\psi}}{2} \\
& +\tilde{r}_{s \psi_{L}} \tilde{m}_{\psi}-\tilde{m}_{\psi} \tilde{r}_{s \psi_{R}}^{\dagger},
\end{aligned}
$$

which results in the following values:

$$
\begin{aligned}
\tilde{c}_{e}^{\prime}= & \frac{3}{4 \pi^{2} \epsilon} \tilde{e}^{2} \tilde{a}_{s} \tilde{m}_{e} \\
& -\frac{1}{16 \pi^{2} \epsilon}\left[\tilde{a}_{e}\left(\tilde{c}_{e}^{\dagger} \tilde{m}_{e}-2 \tilde{m}_{e}^{\dagger} \tilde{c}_{e}\right)+\left(\tilde{m}_{e} \tilde{c}_{e}^{\dagger}-2 \tilde{c}_{e} \tilde{m}_{e}^{\dagger}\right) \tilde{a}_{e}\right] \\
& +\frac{3 \tilde{e}}{8 \pi^{2} \epsilon}\left[\tilde{m}_{e} \tilde{c}_{e}^{\dagger} \tilde{a}_{e A}+\tilde{a}_{e A} \tilde{c}_{e}^{\dagger} \tilde{m}_{e}\right. \\
& \left.-\tilde{c}_{e} \tilde{m}_{e}^{\dagger} \tilde{a}_{e A}-\tilde{a}_{e A} \tilde{m}_{e}^{\dagger} \tilde{c}_{e}\right], \\
\tilde{c}_{u}^{\prime}= & \frac{1}{\pi^{2} \epsilon}\left[\frac{1}{3} \tilde{e}^{2} \tilde{a}_{s}+\tilde{g}_{3}^{2} \tilde{a}_{s \tilde{G}}\right] \tilde{m}_{u} \\
& -\frac{1}{16 \pi^{2} \epsilon}\left[\tilde{a}_{u}\left(\tilde{c}_{u}^{\dagger} \tilde{m}_{u}-2 \tilde{m}_{u}^{\dagger} \tilde{c}_{u}\right)+\left(\tilde{m}_{u} \tilde{c}_{u}^{\dagger}-2 \tilde{c}_{u} \tilde{m}_{u}^{\dagger}\right) \tilde{a}_{u}\right] \\
& -\frac{\tilde{e}}{4 \pi^{2} \epsilon}\left[\tilde{m}_{u} \tilde{c}_{u}^{\dagger} \tilde{a}_{u A}+\tilde{a}_{u A} \tilde{c}_{u}^{\dagger} \tilde{m}_{u}-\tilde{c}_{u} \tilde{m}_{u}^{\dagger} \tilde{a}_{u A}-\tilde{a}_{u A} \tilde{m}_{u}^{\dagger} \tilde{c}_{u}\right] \\
& -\frac{\tilde{g}_{3}}{2 \pi^{2} \epsilon}\left[\tilde{m}_{u} \tilde{c}_{u}^{\dagger} \tilde{a}_{u G}+\tilde{a}_{u G} \tilde{c}_{u}^{\dagger} \tilde{m}_{u}-\tilde{c}_{u} \tilde{m}_{u}^{\dagger} \tilde{a}_{u G}-\tilde{a}_{u G} \tilde{m}_{u}^{\dagger} \tilde{c}_{u}\right], \\
\tilde{c}_{d}^{\prime}= & \frac{1}{4 \pi^{2} \epsilon}\left[\frac{1}{3} \tilde{e}^{2} \tilde{a}_{s} \tilde{A}^{\prime}+4 \tilde{g}_{3}^{2} \tilde{a}_{s \tilde{G}} \tilde{m}_{d} \tilde{m}_{d}\right. \\
& -\frac{1}{16 \pi^{2} \epsilon}\left[\tilde{a}_{d}\left(\tilde{c}_{d}^{\dagger} \tilde{m}_{d}-2 \tilde{m}_{d}^{\dagger} \tilde{c}_{d}\right)+\left(\tilde{m}_{d} \tilde{c}_{d}^{\dagger}-2 \tilde{c}_{d} \tilde{m}_{d}^{\dagger}\right) \tilde{a}_{d}\right] \\
& +\frac{\tilde{e}}{8 \pi^{2} \epsilon}\left[\tilde{m}_{d} \tilde{c}_{d}^{\dagger} \tilde{a}_{d A}+\tilde{a}_{d A} \tilde{c}_{d}^{\dagger} \tilde{m}_{d}-\tilde{c}_{d} \tilde{m}_{d}^{\dagger} \tilde{a}_{d A}-\tilde{a}_{d A} \tilde{m}_{d}^{\dagger} \tilde{c}_{d}\right] \\
& -\frac{\tilde{g}_{3}}{2 \pi^{2} \epsilon}\left[\tilde{m}_{d} \tilde{c}_{d}^{\dagger} \tilde{a}_{d G}+\tilde{a}_{d G} \tilde{c}_{d}^{\dagger} \tilde{m}_{d}-\tilde{c}_{d} \tilde{m}_{d}^{\dagger} \tilde{a}_{d G}-\tilde{a}_{d G} \tilde{m}_{d}^{\dagger} \tilde{c}_{d}\right] .
\end{aligned}
$$


Finally, the redundancies imply the following relations for the coefficients of non-renormalizable operators:

$$
\begin{aligned}
\tilde{a}_{\psi} & \rightarrow \tilde{a}_{\psi}+\tilde{c}_{\psi} \tilde{r}_{\psi \square}^{\dagger} \tilde{c}_{\psi}+\tilde{r}_{s \psi_{L}} \tilde{c}_{\psi}-\tilde{c}_{\psi} \tilde{r}_{s \psi_{R}}^{\dagger}, \\
\tilde{a}_{\psi A} & \rightarrow \tilde{a}_{\psi A}+\frac{\tilde{e} Q_{\psi} \tilde{r}_{\psi \square}}{2}, \\
\tilde{a}_{\psi G} & \rightarrow \tilde{a}_{\psi G}+\frac{\tilde{g}_{3} \tilde{r}_{\psi \square}}{2},
\end{aligned}
$$

resulting in the following on-shell non-renormalizable divergences:

$$
\begin{aligned}
& \tilde{a}_{u}^{\prime}=\left[\frac{1}{9 \pi^{2} \epsilon} \tilde{e}^{2}+\frac{1}{3 \pi \epsilon} \tilde{g}_{3}^{2}-\frac{\tilde{\lambda}_{s}}{32 \pi^{2} \epsilon}\right] \tilde{a}_{u} \\
& +\frac{1}{\pi^{2} \epsilon}\left[\frac{1}{3} \tilde{e}^{2} \tilde{a}_{s \tilde{A}}+\tilde{g}_{3}^{2} \tilde{a}_{s \tilde{G}}\right] \tilde{c}_{u} \\
& -\frac{1}{16 \pi^{2} \epsilon}\left[-\tilde{c}_{u} \tilde{a}_{u}^{\dagger} \tilde{c}_{u}+3 \tilde{a}_{u} \tilde{c}_{u}^{\dagger} \tilde{c}_{u}+3 \tilde{c}_{u} \tilde{c}_{u}^{\dagger} \tilde{a}_{u}\right] \\
& -\frac{1}{4 \pi^{2} \epsilon} \tilde{e}\left[\tilde{c}_{u} \tilde{c}_{u}^{\dagger} \tilde{a}_{u A}+\tilde{a}_{u A} \tilde{c}_{u}^{\dagger} \tilde{c}_{u}\right] \\
& -\frac{\tilde{g}_{3}}{2 \pi^{2} \epsilon}\left[\tilde{c}_{u} \tilde{c}_{u}^{\dagger} \tilde{a}_{u G}+\tilde{a}_{u G} \tilde{c}_{u}^{\dagger} \tilde{c}_{u}\right], \\
& \tilde{a}_{d}^{\prime}=\left[\frac{1}{36 \pi^{2} \epsilon} \tilde{e}^{2}+\frac{1}{3 \pi \epsilon} \tilde{g}_{3}^{2}-\frac{\tilde{\lambda}_{s}}{32 \pi^{2} \epsilon}\right] \tilde{a}_{d} \\
& +\frac{1}{4 \pi^{2} \epsilon}\left[\frac{1}{3} \tilde{e}^{2} \tilde{a}_{s \tilde{A}}+4 \tilde{g}_{3}^{2} \tilde{a}_{s \tilde{G}}\right] \tilde{c}_{d} \\
& -\frac{1}{16 \pi^{2} \epsilon}\left[-\tilde{c}_{d} \tilde{a}_{d}^{\dagger} \tilde{c}_{d}+3 \tilde{a}_{d} \tilde{c}_{d}^{\dagger} \tilde{c}_{d}+3 \tilde{c}_{d} \tilde{c}_{d}^{\dagger} \tilde{a}_{d}\right] \\
& +\frac{1}{8 \pi^{2} \epsilon} \tilde{e}\left[\tilde{c}_{d} \tilde{c}_{d}^{\dagger} \tilde{a}_{d A}+\tilde{a}_{d A} \tilde{c}_{d}^{\dagger} \tilde{c}_{d}\right] \\
& -\frac{\tilde{g}_{3}}{2 \pi^{2} \epsilon}\left[\tilde{c}_{d} \tilde{c}_{d}^{\dagger} \tilde{a}_{d G}+\tilde{a}_{d G} \tilde{c}_{d}^{\dagger} \tilde{c}_{d}\right] \text {, } \\
& \tilde{a}_{e}^{\prime}=\left[\frac{1}{4 \pi^{2} \epsilon} \tilde{e}^{2}-\frac{\tilde{\lambda}_{s}}{32 \pi^{2} \epsilon}\right] \tilde{a}_{e}+\frac{3}{4 \pi^{2} \epsilon} \tilde{e}^{2} \tilde{a}_{s} \tilde{A}_{e} \\
& -\frac{1}{16 \pi^{2} \epsilon}\left[-\tilde{c}_{e} \tilde{a}_{e}^{\dagger} \tilde{c}_{e}+3 \tilde{a}_{e} \tilde{c}_{e}^{\dagger} \tilde{c}_{e}+3 \tilde{c}_{e} \tilde{c}_{e}^{\dagger} \tilde{a}_{e}\right] \\
& +\frac{3}{8 \pi^{2} \epsilon} \tilde{e}\left[\tilde{c}_{e} \tilde{c}_{e}^{\dagger} \tilde{a}_{e A}+\tilde{a}_{e A} \tilde{c}_{e}^{\dagger} \tilde{c}_{e}\right] \text {, } \\
& \tilde{a}_{e A}^{\prime}=-\frac{\tilde{e}}{8 \pi^{2} \epsilon}\left[2 \tilde{e} \tilde{a}_{e A}+\tilde{c}_{e} \tilde{a}_{s \tilde{A}}\right] \text {, } \\
& \tilde{a}_{u A}^{\prime}=-\frac{\tilde{e}}{12 \pi^{2} \epsilon}\left[\frac{4}{3} \tilde{e} \tilde{a}_{u A}-\tilde{c}_{u} \tilde{a}_{s \tilde{A}}\right]-\frac{2 \tilde{e}}{9 \pi^{2} \epsilon} \tilde{g}_{3} \tilde{a}_{u \tilde{G}}, \\
& \tilde{a}_{d A}^{\prime}=-\frac{\tilde{e}}{24 \pi^{2} \epsilon}\left[\frac{2}{3} \tilde{e} \tilde{a}_{d A}+\tilde{c}_{d} \tilde{a}_{s \tilde{A}}\right]+\frac{\tilde{e}}{9 \pi^{2} \epsilon} \tilde{g}_{3} \tilde{a}_{d \tilde{G}}, \\
& \tilde{a}_{u G}^{\prime}=-\frac{\tilde{g}_{3}}{8 \pi^{2} \epsilon}\left[\frac{4}{3} \tilde{e} \tilde{a}_{u A}-\tilde{c}_{u} \tilde{a}_{s \tilde{G}}\right]+\frac{1}{24 \pi^{2} \epsilon} \tilde{g}_{3}^{2} \tilde{a}_{u G}, \\
& \tilde{a}_{d G}^{\prime}=\frac{\tilde{g}_{3}}{8 \pi^{2} \epsilon}\left[\frac{2}{3} \tilde{e} \tilde{a}_{d A}+\tilde{c}_{d} \tilde{a}_{s \tilde{G}}\right]+\frac{1}{24 \pi^{2} \epsilon} \tilde{g}_{3}^{2} \tilde{a}_{d G} .
\end{aligned}
$$

\subsection{Anomalous dimensions}

Once we have parametrised all the relevant divergences in the on-shell basis, we can obtain the beta functions of the different parameters following the standard procedure outlined in Sect. 4. We start reporting the beta functions for the renormalizable couplings, which read:

$$
\begin{aligned}
\beta_{\tilde{e}}= & \frac{4}{3}\left[n_{\ell}+\frac{1}{3} n_{d}+\frac{4}{3} n_{u}\right] \tilde{e}^{3} \\
& +8 \tilde{e}^{2} \operatorname{Tr}\left[-\left(\tilde{a}_{e A}^{\dagger} \tilde{m}_{e}+\tilde{m}_{e}^{\dagger} \tilde{a}_{e A}\right)+2\left(\tilde{a}_{u A}^{\dagger} \tilde{m}_{u}+\tilde{m}_{u}^{\dagger} \tilde{a}_{u A}\right)\right. \\
& \left.-\left(\tilde{a}_{d A}^{\dagger} \tilde{m}_{d}+\tilde{m}_{d}^{\dagger} \tilde{a}_{d A}\right)\right] \\
\beta_{\tilde{g}_{3}=} & {\left[-11+\frac{2}{3}\left(n_{u}+n_{d}\right)\right] \tilde{g}_{3}^{3}+4 \tilde{g}_{3}^{2} \operatorname{Tr} } \\
& \times\left[\tilde{a}_{d G}^{\dagger} \tilde{m}_{d}+\tilde{m}_{d}^{\dagger} \tilde{a}_{d G}+\tilde{a}_{u G}^{\dagger} \tilde{m}_{u}+\tilde{m}_{u}^{\dagger} \tilde{a}_{u G}\right] \\
\beta_{\tilde{m}_{e}=} & -6 \tilde{e}^{2} \tilde{m}_{e}+\frac{1}{2}\left(\tilde{m}_{e} \tilde{c}_{e}^{\dagger} \tilde{c}_{e}+\tilde{c}_{e} \tilde{c}_{e}^{\dagger} \tilde{m}_{e}+4 \tilde{c}_{e} \tilde{m}_{e}^{\dagger} \tilde{c}_{e}\right) \\
& +\operatorname{Tr}\left(\tilde{c}_{e} \tilde{m}_{e}^{\dagger}+\tilde{c}_{e}^{\dagger} \tilde{m}_{e}+3 \tilde{c}_{u} \tilde{m}_{u}^{\dagger}+3 \tilde{c}_{u}^{\dagger} \tilde{m}_{u}\right. \\
& \left.+3 \tilde{m}_{d} \tilde{c}_{d}^{\dagger}+3 \tilde{m}_{d}^{\dagger} \tilde{c}_{d}\right) \tilde{c}_{e} \\
& +12 \tilde{e}\left(\tilde{m}_{e} \tilde{m}_{e}^{\dagger} \tilde{a}_{e A}+\tilde{a}_{e A} \tilde{m}_{e}^{\dagger} \tilde{m}_{e}\right)-2 \tilde{m}^{2} \tilde{a}_{e}, \\
\beta_{\tilde{m}_{u}=} & -8 \tilde{g}_{3}^{2} \tilde{m}_{u}-\frac{8}{3} \tilde{e}^{2} \tilde{m}_{u} \\
+ & \frac{1}{2}\left(\tilde{m}_{u} \tilde{c}_{u}^{\dagger} \tilde{c}_{u}+\tilde{c}_{u} \tilde{c}_{u}^{\dagger} \tilde{m}_{u}+4 \tilde{c}_{u} \tilde{m}_{u}^{\dagger} \tilde{c}_{u}\right) \\
+ & \operatorname{Tr}\left(\tilde{c}_{e} \tilde{m}_{e}^{\dagger}+\tilde{c}_{e}^{\dagger} \tilde{m}_{e}+3 \tilde{c}_{u} \tilde{m}_{u}^{\dagger}+3 \tilde{c}_{u}^{\dagger} \tilde{m}_{u}+3 \tilde{m}_{u}^{\dagger} \tilde{a}_{u A}+\tilde{a}_{u A} \tilde{m}_{u}^{\dagger} \tilde{m}_{u}\right) \\
- & \left.16 \tilde{g}_{3}\left(\tilde{m}_{u} \tilde{m}_{u}^{\dagger} \tilde{a}_{u G}+\tilde{a}_{u G} \tilde{m}_{u}^{\dagger} \tilde{m}_{u}\right)-2 \tilde{m}_{d}^{\dagger} \tilde{c}_{d}\right) \tilde{c}_{u} \\
& (148) \\
& \\
&
\end{aligned}
$$

$$
\begin{aligned}
\beta_{\tilde{m}_{d}}= & -8 \tilde{g}_{3}^{2} \tilde{m}_{d}-\frac{2}{3} \tilde{e}^{2} \tilde{m}_{d} \\
& +\frac{1}{2}\left(\tilde{m}_{d} \tilde{c}_{d}^{\dagger} \tilde{c}_{d}+\tilde{c}_{d} \tilde{c}_{d}^{\dagger} \tilde{m}_{d}+4 \tilde{c}_{d} \tilde{m}_{d}^{\dagger} \tilde{c}_{d}\right) \\
& +\operatorname{Tr}\left(\tilde{c}_{e} \tilde{m}_{e}^{\dagger}+\tilde{c}_{e}^{\dagger} \tilde{m}_{e}+3 \tilde{c}_{u} \tilde{m}_{u}^{\dagger}\right. \\
& \left.+3 \tilde{c}_{u}^{\dagger} \tilde{m}_{u}+3 \tilde{m}_{d} \tilde{c}_{d}^{\dagger}+3 \tilde{m}_{d}^{\dagger} \tilde{c}_{d}\right) \tilde{c}_{u} \\
& \times 4 \tilde{e}\left(\tilde{m}_{d} \tilde{m}_{d}^{\dagger} \tilde{a}_{d A}+\tilde{a}_{d A} \tilde{m}_{d}^{\dagger} \tilde{m}_{d}\right) \\
& -16 \tilde{g}_{3}\left(\tilde{m}_{d} \tilde{m}_{d}^{\dagger} \tilde{a}_{d G}+\tilde{a}_{d G} \tilde{m}_{d}^{\dagger} \tilde{m}_{d}\right)-2 \tilde{m}^{2} \tilde{a}_{d}, \\
\beta_{\tilde{m}^{2}}= & \tilde{\lambda}_{s} \tilde{m}^{2}+4 \tilde{m}^{2} \operatorname{Tr}\left(\tilde{c}_{e} \tilde{c}_{e}^{\dagger}\right) \\
& +12 \tilde{m}^{2} \operatorname{Tr}\left(\tilde{c}_{d} \tilde{c}_{d}^{\dagger}\right)+12 \tilde{m}^{2} \operatorname{Tr}\left(\tilde{c}_{u} \tilde{c}_{u}^{\dagger}\right) \\
& -24 \operatorname{Tr}\left(\tilde{c}_{u} \tilde{c}_{u}^{\dagger} \tilde{m}_{u} \tilde{m}_{u}^{\dagger}+\tilde{c}_{d} \tilde{c}_{d}^{\dagger} \tilde{m}_{d} \tilde{m}_{d}^{\dagger}\right) \\
& -18 \operatorname{Tr}\left(\tilde{c}_{u}^{\dagger} \tilde{c}_{u} \tilde{m}_{u}^{\dagger} \tilde{m}_{u}+\tilde{c}_{d}^{\dagger} \tilde{c}_{d} \tilde{m}_{d}^{\dagger} \tilde{m}_{d}\right) \\
& -12 \operatorname{Tr}\left(\tilde{c}_{u} \tilde{m}_{u}^{\dagger} \tilde{c}_{u} \tilde{m}_{u}^{\dagger}+\tilde{c}_{u}^{\dagger} \tilde{m}_{u} \tilde{c}_{u}^{\dagger} \tilde{m}_{u}\right. \\
& \left.+\tilde{c}_{d} \tilde{m}_{d}^{\dagger} \tilde{c}_{d} \tilde{m}_{d}^{\dagger}+\tilde{c}_{d}^{\dagger} \tilde{m}_{d} \tilde{c}_{d}^{\dagger} \tilde{m}_{d}\right)
\end{aligned}
$$


$-6 \operatorname{Tr}\left(\tilde{c}_{u} \tilde{m}_{u}^{\dagger} \tilde{m}_{u} \tilde{c}_{u}^{\dagger}+\tilde{m}_{d} \tilde{c}_{d}^{\dagger} \tilde{c}_{d} \tilde{m}_{d}^{\dagger}+\tilde{c}_{e}^{\dagger} \tilde{c}_{e} \tilde{m}_{e}^{\dagger} \tilde{m}_{e}\right)$

$-2 \operatorname{Tr}\left(4 \tilde{c}_{e} \tilde{c}_{e}^{\dagger} \tilde{m}_{e} \tilde{m}_{e}^{\dagger}+2 \tilde{c}_{e} \tilde{m}_{e}^{\dagger} \tilde{c}_{e} \tilde{m}_{e}^{\dagger}\right.$

$\left.+2 \tilde{c}_{e}^{\dagger} \tilde{m}_{e} \tilde{c}_{e}^{\dagger} \tilde{m}_{e}+\tilde{c}_{e} \tilde{m}_{e}^{\dagger} \tilde{m}_{e} \tilde{c}_{e}^{\dagger}\right)$

$+8\left[3 \operatorname{Tr}\left(\tilde{m}_{d}^{\dagger} \tilde{a}_{d} \tilde{m}_{d}^{\dagger} \tilde{m}_{d}+\tilde{m}_{d}^{\dagger} \tilde{m}_{d} \tilde{a}_{d}^{\dagger} \tilde{m}_{d}\right)\right.$

$+3 \operatorname{Tr}\left(\tilde{m}_{u}^{\dagger} \tilde{a}_{u} \tilde{m}_{u}^{\dagger} \tilde{m}_{u}+\tilde{m}_{u}^{\dagger} \tilde{m}_{u} \tilde{a}_{u}^{\dagger} \tilde{m}_{u}\right)$

$\left.+\operatorname{Tr}\left(\tilde{m}_{e}^{\dagger} \tilde{a}_{e} \tilde{m}_{e}^{\dagger} \tilde{m}_{e}+\tilde{m}_{e}^{\dagger} \tilde{m}_{e} \tilde{a}_{e}^{\dagger} \tilde{m}_{e}\right)\right]$

$$
\begin{aligned}
\beta_{\tilde{\lambda}_{s}}= & 3 \tilde{\lambda}_{s}^{2}-144 \operatorname{Tr}\left(\tilde{c}_{d} \tilde{c}_{d}^{\dagger} \tilde{c}_{d} \tilde{c}_{d}^{\dagger}\right) \\
& -144 \operatorname{Tr}\left(\tilde{c}_{u} \tilde{c}_{u}^{\dagger} \tilde{c}_{u} \tilde{c}_{u}^{\dagger}\right)-48 \operatorname{Tr}\left(\tilde{c}_{e} \tilde{c}_{e}^{\dagger} \tilde{c}_{e} \tilde{c}_{e}^{\dagger}\right) \\
& +24 \tilde{\lambda}_{s} \operatorname{Tr}\left(\tilde{c}_{d} \tilde{c}_{d}^{\dagger}\right)+24 \tilde{\lambda}_{s} \operatorname{Tr}\left(\tilde{c}_{u} \tilde{c}_{u}^{\dagger}\right) \\
& +8 \tilde{\lambda}_{s} \operatorname{Tr}\left(\tilde{c}_{e} \tilde{c}_{e}^{\dagger}\right)+96\left[\operatorname{Tr}_{e}^{\tilde{\lambda}_{e}}+3\left(\operatorname{Tr}_{u}^{\tilde{\lambda}_{u}}+\operatorname{Tr}_{d}^{\tilde{\lambda}}\right)\right],
\end{aligned}
$$

$$
\begin{aligned}
\beta_{\tilde{c}_{u}}= & -\frac{24}{9}\left(\tilde{e}^{2}+3 \tilde{g}_{3}^{2}\right) \tilde{c}_{u}+3 \tilde{c}_{u} \tilde{c}_{u}^{\dagger} \tilde{c}_{u} \\
& +2\left[\operatorname{Tr}\left(\tilde{c}_{e} \tilde{c}_{e}^{\dagger}\right)+3 \operatorname{Tr}\left(\tilde{c}_{d} \tilde{c}_{d}^{\dagger}\right)+3 \operatorname{Tr}\left(\tilde{c}_{u} \tilde{c}_{u}^{\dagger}\right)\right] \tilde{c}_{u}+ \\
& -32\left[\frac{1}{3} \tilde{e}^{2} \tilde{a}_{s \tilde{A}}+\tilde{g}_{3}^{2} \tilde{a}_{s \tilde{G}}\right] \tilde{m}_{u} \\
& +2\left[\tilde{a}_{u}\left(\tilde{c}_{u}^{\dagger} \tilde{m}_{u}-2 \tilde{m}_{u}^{\dagger} \tilde{c}_{u}\right)+\left(\tilde{m}_{u} \tilde{c}_{u}^{\dagger}-2 \tilde{c}_{u} \tilde{m}_{u}^{\dagger}\right) \tilde{a}_{u}\right] \\
& +8 \tilde{e}\left[\tilde{m}_{u} \tilde{c}_{u}^{\dagger} \tilde{a}_{u A}+\tilde{a}_{u A} \tilde{c}_{u}^{\dagger} \tilde{m}_{u}-\tilde{c}_{u} \tilde{m}_{u}^{\dagger} \tilde{a}_{u A}-\tilde{a}_{u A} \tilde{m}_{u}^{\dagger} \tilde{c}_{u}\right] \\
& +16 \tilde{g}_{3}\left[\tilde{m}_{u} \tilde{c}_{u}^{\dagger} \tilde{a}_{u G}+\tilde{a}_{u G} \tilde{c}_{u}^{\dagger} \tilde{m}_{u}-\tilde{c}_{u} \tilde{m}_{u}^{\dagger} \tilde{a}_{u G}-\tilde{a}_{u G} \tilde{m}_{u}^{\dagger} \tilde{c}_{u}\right],
\end{aligned}
$$

$$
\begin{aligned}
\beta_{\tilde{c}_{d}}= & -\frac{2}{3}\left(\tilde{e}^{2}+12 \tilde{g}_{3}^{2}\right) \tilde{c}_{d}+3 \tilde{c}_{d} \tilde{c}_{d}^{\dagger} \tilde{c}_{d} \\
& +2\left[\operatorname{Tr}\left(\tilde{c}_{e} \tilde{c}_{e}^{\dagger}\right)+3 \operatorname{Tr}\left(\tilde{c}_{d} \tilde{c}_{d}^{\dagger}\right)+3 \operatorname{Tr}\left(\tilde{c}_{u} \tilde{c}_{u}^{\dagger}\right)\right] \tilde{c}_{d} \\
& -8\left[\frac{1}{3} \tilde{e}^{2} \tilde{a}_{s \tilde{A}}+4 \tilde{g}_{3}^{2} \tilde{a}_{s} \tilde{G}\right] \tilde{m}_{d} \\
& +2\left[\tilde{a}_{d}\left(\tilde{c}_{d}^{\dagger} \tilde{m}_{d}-2 \tilde{m}_{d}^{\dagger} \tilde{c}_{d}\right)+\left(\tilde{m}_{d} \tilde{c}_{d}^{\dagger}-2 \tilde{c}_{d} \tilde{m}_{d}^{\dagger}\right) \tilde{a}_{d}\right] \\
& -4 \tilde{e}\left[\tilde{m}_{d} \tilde{c}_{d}^{\dagger} \tilde{a}_{d A}+\tilde{a}_{d A} \tilde{c}_{d}^{\dagger} \tilde{m}_{d}-\tilde{c}_{d} \tilde{m}_{d}^{\dagger} \tilde{a}_{d A}-\tilde{a}_{d A} \tilde{m}_{d}^{\dagger} \tilde{c}_{d}\right] \\
& +16 \tilde{g}_{3}\left[\tilde{m}_{d} \tilde{c}_{d}^{\dagger} \tilde{a}_{d G}+\tilde{a}_{d G} \tilde{c}_{d}^{\dagger} \tilde{m}_{d}-\tilde{c}_{d} \tilde{m}_{d}^{\dagger} \tilde{a}_{d G}-\tilde{a}_{d G} \tilde{m}_{d}^{\dagger} \tilde{c}_{d}\right],
\end{aligned}
$$

$$
\begin{aligned}
\beta_{\tilde{c}_{e}}= & -6 \tilde{e}^{2} \tilde{c}_{e}+3 \tilde{c}_{e} \tilde{c}_{e}^{\dagger} \tilde{c}_{e}+2\left[\operatorname{Tr}\left(\tilde{c}_{e} \tilde{c}_{e}^{\dagger}\right)\right. \\
& \left.+6 \operatorname{Tr}\left(\tilde{c}_{d} \tilde{c}_{d}^{\dagger}\right)+6 \operatorname{Tr}\left(\tilde{c}_{u} \tilde{c}_{u}^{\dagger}\right)\right] \tilde{c}_{e} \\
& -8\left[3 \tilde{e}^{2} \tilde{a}_{s} \tilde{A}\right] \tilde{m}_{e}+2\left[\tilde{a}_{e}\left(\tilde{c}_{e}^{\dagger} \tilde{m}_{e}-2 \tilde{m}_{e}^{\dagger} \tilde{c}_{e}\right)\right. \\
& \left.+\left(\tilde{m}_{e} \tilde{c}_{e}^{\dagger}-2 \tilde{c}_{e} \tilde{m}_{e}^{\dagger}\right) \tilde{a}_{e}\right]
\end{aligned}
$$

$$
-12 \tilde{e}\left[\tilde{m}_{e} \tilde{c}_{e}^{\dagger} \tilde{a}_{e A}+\tilde{a}_{e A} \tilde{c}_{e}^{\dagger} \tilde{m}_{e}-\tilde{c}_{e} \tilde{m}_{e}^{\dagger} \tilde{a}_{e A}-\tilde{a}_{e A} \tilde{m}_{e}^{\dagger} \tilde{c}_{e}\right]
$$

where the contributions from the effective operators are apparent from the presence of the corresponding Wilson coefficients, and we have used Pyr@te [72] with manual cross-checks to compute the part of the beta functions that depend only on renormalizable couplings.

As a byproduct of this work, we have reproduced the anomalous dimensions of purely SM operators to dimension five given in Ref. [73]. One can also trivially reproduce the $\log \left(m_{W} / m_{\psi}\right)$ piece of the ALP-fermion-fermion couplings induced by ALP-vector-vector ones in Eqs. 3.15 and 3.20 of Ref. [24].

In the case of the non-renormalizable Wilson coefficients, the beta functions read:

$$
\begin{aligned}
\beta_{\tilde{a}_{u}}= & {\left[-\frac{8}{3} \tilde{e}^{2}-8 \tilde{g}_{3}^{2}+\tilde{\lambda}_{s}\right] \tilde{a}_{u} } \\
& -32\left[\frac{1}{3} \tilde{e}^{2} \tilde{a}_{s} \tilde{A}+\tilde{g}_{3}^{2} \tilde{a}_{s} \tilde{G}\right] \tilde{c}_{u} \\
& +2\left[-\tilde{c}_{u} \tilde{a}_{u}^{\dagger} \tilde{c}_{u}+\frac{13}{4} \tilde{a}_{u} \tilde{c}_{u}^{\dagger} \tilde{c}_{u}+\frac{13}{4} \tilde{c}_{u} \tilde{c}_{u}^{\dagger} \tilde{a}_{u}\right] \\
& +4 \operatorname{Tr}\left[\tilde{c}_{e} \tilde{c}_{e}^{\dagger}+3\left(\tilde{c}_{d} \tilde{c}_{d}^{\dagger}+\tilde{c}_{u} \tilde{c}_{u}^{\dagger}\right)\right] \tilde{a}_{u} \\
& +8 \tilde{e}\left[\tilde{c}_{u} \tilde{c}_{u}^{\dagger} \tilde{a}_{u A}+\tilde{a}_{u A} \tilde{c}_{u}^{\dagger} \tilde{c}_{u}\right] \\
& +16 \tilde{g}_{3}\left[\tilde{c}_{u} \tilde{c}_{u}^{\dagger} \tilde{a}_{u G}+\tilde{a}_{u G} \tilde{c}_{u}^{\dagger} \tilde{c}_{u}\right],
\end{aligned}
$$

$$
\begin{aligned}
\beta_{\tilde{a}_{d}}= & {\left[-\frac{2}{3} \tilde{e}^{2}-8 \tilde{g}_{3}^{2}+\tilde{\lambda}_{s}\right] \tilde{a}_{d} } \\
& -32\left[\frac{1}{12} \tilde{e}^{2} \tilde{a}_{s \tilde{A}}+\tilde{g}_{3}^{2} \tilde{a}_{s} \tilde{\sigma}_{\tilde{c}}\right] \tilde{c}_{d} \\
& +2\left[-\tilde{c}_{d} \tilde{a}_{d}^{\dagger} \tilde{c}_{d}+\frac{13}{4} \tilde{a}_{d} \tilde{c}_{d}^{\dagger} \tilde{c}_{d}+\frac{13}{4} \tilde{c}_{d} \tilde{c}_{d}^{\dagger} \tilde{a}_{d}\right] \\
& +4 \operatorname{Tr}\left[\tilde{c}_{e} \tilde{c}_{e}^{\dagger}+3\left(\tilde{c}_{d} \tilde{c}_{d}^{\dagger}+\tilde{c}_{u} \tilde{c}_{u}^{\dagger}\right)\right] \tilde{a}_{d} \\
& -4 \tilde{e}\left[\tilde{c}_{d} \tilde{c}_{d}^{\dagger} \tilde{a}_{d A}+\tilde{a}_{d A} \tilde{c}_{d}^{\dagger} \tilde{c}_{d}\right] \\
& +16 \tilde{g}_{3}\left[\tilde{c}_{d} \tilde{c}_{d}^{\dagger} \tilde{a}_{d G}+\tilde{a}_{d G} \tilde{c}_{d}^{\dagger} \tilde{c}_{d}\right],
\end{aligned}
$$

$$
\begin{aligned}
\beta_{\tilde{a}_{e}}= & {\left[-6 \tilde{e}^{2}+\tilde{\lambda}_{s}\right] \tilde{a}_{e}-24 \tilde{e}^{2} \tilde{a}_{s \tilde{A}} \tilde{c}_{e} } \\
& -12 \tilde{e}\left[\tilde{c}_{e} \tilde{c}_{e}^{\dagger} \tilde{a}_{e A}+\tilde{a}_{e A} \tilde{c}_{e}^{\dagger} \tilde{c}_{e}\right] \\
& +2\left[-\tilde{c}_{e} \tilde{a}_{e}^{\dagger} \tilde{c}_{e}+\frac{13}{4} \tilde{a}_{e} \tilde{c}_{e}^{\dagger} \tilde{c}_{e}+\frac{13}{4} \tilde{c}_{e} \tilde{c}_{e}^{\dagger} \tilde{a}_{e}\right] \\
& +4 \operatorname{Tr}\left[\tilde{c}_{e} \tilde{c}_{e}^{\dagger}+3\left(\tilde{c}_{d} \tilde{c}_{d}^{\dagger}+\tilde{c}_{u} \tilde{c}_{u}^{\dagger}\right)\right] \tilde{a}_{e},
\end{aligned}
$$




$$
\begin{aligned}
& \beta_{\tilde{a}_{s \widetilde{A}}}=4 \tilde{e} \operatorname{Tr}\left[\left(\tilde{c}_{e} \tilde{a}_{e A}^{\dagger}+\tilde{c}_{e}^{\dagger} \tilde{a}_{e A}\right)+\left(\tilde{c}_{d} \tilde{a}_{d A}^{\dagger}+\tilde{c}_{d}^{\dagger} \tilde{a}_{d A}\right)\right. \\
& \left.-2\left(\tilde{c}_{u} \tilde{a}_{u A}^{\dagger}+\tilde{c}_{u}^{\dagger} \tilde{a}_{u A}\right)\right] \\
& +2 \operatorname{Tr}\left[\tilde{c}_{e} \tilde{c}_{e}^{\dagger}+3\left(\tilde{c}_{d} \tilde{c}_{d}^{\dagger}+\tilde{c}_{u} \tilde{c}_{u}^{\dagger}\right)\right] \tilde{a}_{s \tilde{A}} \\
& +\frac{8}{3} \tilde{e}^{2}\left[n_{\ell}+\frac{1}{3} n_{d}+\frac{4}{3} n_{u}\right] \tilde{a}_{s \tilde{A}}, \\
& \beta_{\tilde{a}_{s \widetilde{G}}}=-2 \tilde{g}_{3} \operatorname{Tr}\left[\tilde{c}_{d} \tilde{a}_{d G}^{\dagger}+\tilde{c}_{d}^{\dagger} \tilde{a}_{d G}+\tilde{c}_{u} \tilde{a}_{u G}^{\dagger}+\tilde{c}_{u}^{\dagger} \tilde{a}_{u G}\right] \\
& +2 \operatorname{Tr}\left[\tilde{c}_{e} \tilde{c}_{e}^{\dagger}+3\left(\tilde{c}_{d} \tilde{c}_{d}^{\dagger}+\tilde{c}_{u} \tilde{c}_{u}^{\dagger}\right)\right] \tilde{a}_{s \tilde{G}} \\
& +2 \tilde{g}_{3}^{2}\left[\frac{2}{3}\left(n_{u}+n_{d}\right)-11\right] \tilde{a}_{s \tilde{G}} \\
& \beta_{\tilde{a}_{e A}}=10 \tilde{e}^{2} \tilde{a}_{e A}+4 \tilde{e} \tilde{c}_{e} \tilde{a}_{s \tilde{A}}+\frac{1}{2}\left(\tilde{c}_{e} \tilde{c}_{e}^{\dagger} \tilde{a}_{e A}+\tilde{a}_{e A} \tilde{c}_{e}^{\dagger} \tilde{c}_{e}\right) \\
& +\frac{4}{3} \tilde{e}^{2}\left[n_{\ell}+\frac{1}{3} n_{d}+\frac{4}{3} n_{u}\right] \tilde{a}_{e A}, \\
& \beta_{\tilde{a}_{u A}}=\frac{40}{9} \tilde{e}^{2} \tilde{a}_{u A}-\frac{8}{3} \tilde{e} \tilde{c}_{u} \tilde{a}_{s} \tilde{A}+\frac{64}{9} \tilde{e} \tilde{g}_{3} \tilde{a}_{u G}+\frac{8}{3} \tilde{g}_{3}^{2} \tilde{a}_{u A} \\
& +\frac{1}{2}\left(\tilde{c}_{u} \tilde{c}_{u}^{\dagger} \tilde{a}_{u A}+\tilde{a}_{u A} \tilde{c}_{u}^{\dagger} \tilde{c}_{u}\right) \\
& +\frac{4}{3} \tilde{e}^{2}\left[n_{\ell}+\frac{1}{3} n_{d}+\frac{4}{3} n_{u}\right] \tilde{a}_{u A}, \\
& \beta_{\tilde{a}_{d A}}=\frac{10}{9} \tilde{e}^{2} \tilde{a}_{d A}+\frac{4}{3} \tilde{e} \tilde{c}_{d} \tilde{a}_{s} \tilde{A}-\frac{32 \tilde{e}}{9} \tilde{g}_{3} \tilde{a}_{d G}+\frac{8}{3} \tilde{g}_{3}^{2} \tilde{a}_{d A} \\
& +\frac{1}{2}\left(\tilde{c}_{d} \tilde{c}_{d}^{\dagger} \tilde{a}_{d A}+\tilde{a}_{d A} \tilde{c}_{d}^{\dagger} \tilde{c}_{d}\right) \\
& +\frac{4}{3} \tilde{e}^{2}\left[n_{\ell}+\frac{1}{3} n_{d}+\frac{4}{3} n_{u}\right] \tilde{a}_{d A}, \\
& \beta_{\tilde{a}_{u G}}=\frac{16}{3} \tilde{g}_{3} \tilde{e} \tilde{a}_{u A}-4 \tilde{g}_{3} \tilde{c}_{u} \tilde{a}_{s \tilde{G}}+\frac{8}{9} \tilde{e}^{2} \tilde{a}_{u G} \\
& +\frac{1}{2}\left(\tilde{c}_{u} \tilde{c}_{u}^{\dagger} \tilde{a}_{u G}+\tilde{a}_{u G} \tilde{c}_{u}^{\dagger} \tilde{c}_{u}\right) \\
& +\frac{1}{3}\left[2\left(n_{u}+n_{d}\right)-29\right] \tilde{g}_{3}^{2} \tilde{a}_{u G}, \\
& \beta_{\tilde{a}_{d G}}=-\frac{8}{3} \tilde{g}_{3} \tilde{e} \tilde{a}_{d A}-4 \tilde{g}_{3} \tilde{c}_{d}^{1} \tilde{a}_{s \tilde{G}}+\frac{2}{9} \tilde{e}^{2} \tilde{a}_{d G} \\
& +\frac{1}{2}\left(\tilde{c}_{d} \tilde{c}_{d}^{\dagger} \tilde{a}_{d G}+\tilde{a}_{d G} \tilde{c}_{d}^{\dagger} \tilde{c}_{d}\right) \\
& +\frac{1}{3}\left[2\left(n_{u}+n_{d}\right)-29\right] \tilde{g}_{3}^{2} \tilde{a}_{d G} ;
\end{aligned}
$$

where $n_{u}, n_{d}$ and $n_{e}$ are the number of dynamical up-type quarks, down-type quarks and charged leptons, respectively, for the EFT we are considering. The appearance of fermionic dipole moments induced by ALP couplings [50] is manifest.

The equations above are fully generic, meaning that they hold irrespective of whether the EFT in the UV is the one we have assumed in Sect. 2 and that leads to the matching conditions in Eqs. (74)-(79), or rather any other EFT containing different degrees of freedom such as for example a second scalar doublet with arbitrary Yukawa couplings which would lead to non vanishing $\tilde{a}_{u, d, e}$. In the former case, though, one should take into account that $\tilde{c}_{u, d, e}$ are already $1 / \Lambda$ suppressed and therefore terms with more than one appearance of these Wilson coefficients should be neglected for consistency.

Within our EFT(s), the parameters $\tilde{a}_{u}, \tilde{a}_{d}$ and $\tilde{a}_{e}$ vanish at all scales, and the renormalizable fermion and ALP masses do not get contributions from dimension-five operators. In general this is not the case and we have, for example,

$\delta \tilde{m}^{2} \sim \tilde{m}_{e}^{3} \tilde{a}_{e}, \quad \delta \tilde{m}_{e} \sim \tilde{m}^{2} \tilde{a}_{e}$

and likewise for quarks.

The running of the operators involving coloured particles should not be taken at face value at energies close or below $\Lambda_{Q C D} \sim 200 \mathrm{MeV}$, where QCD becomes strongly coupled. Otherwise, the equations above, together with Eqs. (51)(54), can be used to make predictions within the ALP EFT to leading-log accuracy across all energy scales.

\section{Some phenomenological applications}

The mixing of different operators can have a significant impact in the understanding of extensions of the SM with ALPs. To exemplify this point, we consider in this section the following simple Lagrangian, defined at the scale $\Lambda=10 \mathrm{TeV}$ :

$$
\begin{aligned}
\mathcal{L}= & \mathcal{L}_{\mathrm{SM}}+\frac{1}{2} \partial_{\mu} s \partial^{\mu} s+\frac{1}{2} \tilde{m}^{2} s^{2} \\
& +\frac{a_{s} \widetilde{Z}}{c_{\omega}^{2}-s_{\omega}^{2}} s\left(c_{\omega}^{2} W_{\mu \nu} \widetilde{W}^{\mu \nu}-s_{\omega}^{2} B_{\mu \nu} \widetilde{B}^{\mu \nu}\right),
\end{aligned}
$$

where $\mathcal{L}_{\mathrm{SM}}$ stands for the SM Lagrangian. In this Lagrangian, the ALP couples to pairs of $Z$ bosons but not to pairs of photons. Despite its simplicity, this structure arises for example in the next-to-minimal composite Higgs model based on $S O(6) / S O(5)$ as a result of quantum anomalies [6]. Within this framework, the photophobic condition is stable, namely $a_{s \tilde{A}}$ remains vanishing at all scales. (The ALP can still couple to photons proportionally to $\tilde{m}$ via loops of heavy gauge bosons [25], for instance.) However, below the EW scale, the ALP coupling to photons could be induced by (purely SM) dipole operators even at dimension five if the high-energy theory is not just the ALP EFT, but it rather involves other states near the EW scale.

Let us assume that the physical ALP mass is $\mathcal{O}(\mathrm{KeV})$. While $a_{s} \widetilde{Z}$ can be directly constrained at colliders, e.g. in $p p \rightarrow Z s$, the corresponding bounds are very weak. For example, values of $a_{s} \tilde{Z}$ larger than $0.2 \mathrm{TeV}^{-1}$ can be bound, depending on the value of $\tilde{m}$, from LHC Run II data [27]. This bound can be extended to values above $0.04 \mathrm{TeV}^{-1}$ for the High-Luminosity phase of the LHC [27]. However, $a_{s} \widetilde{Z}$ 
does generate, through mixing, other operators with nonvanishing Wilson coefficients, in particular $a_{s e \phi}$, which is very constrained experimentally. Indeed, the most stringent bound for ALPs with masses $\sim \mathrm{KeV}$ comes from the modification of Red Giants cooling due to ALP radiation. This sets a bound on the ALP coupling to electrons $\tilde{c}_{e} \lesssim 3 \times 10^{-13}$, for a typical core temperature of $T \approx 10^{8} \mathrm{~K}$ [42]. Since we are assuming that there are no degrees of freedom other than the ALP and the SM ones below $\Lambda, \tilde{c}_{e}$ runs proportional to itself. Resumming Eqs. (145) and (154), including $\mathcal{O}(1 / \Lambda)$ effects, we obtain at the EW scale:

$\tilde{c}_{e}(v) \lesssim 2.8 \times 10^{-13}$,

which translates into

$a_{\text {se申 }}(v) \lesssim 1.6 \times 10^{-12} \mathrm{TeV}^{-1}$.

Solving numerically Eqs. (51)-(56), (199)-(201) and (207)(209) for $\lambda_{s \phi}=0$, we can compute the maximum allowed value for $a_{s} \widetilde{Z}(10 \mathrm{TeV})$ :

$a_{s \widetilde{Z}}(10 \mathrm{TeV}) \lesssim 4.8 \times 10^{-6} \mathrm{TeV}^{-1}$.

Despite the electron Yukawa suppression, this is four orders of magnitude stronger than prospects from direct searches. Such analysis of the photophobic ALP was previously performed in Ref. [25] by considering solely the running of the gauge operators. As shown in Eq. (167) the running from the EW scale down to the $\mathrm{KeV}$ amounts to a $\sim 6 \%$ effect, which can be taken as a systematic theory error when using only the EFT before EWSB. See also Ref. [75] for an analysis of the RGE effects in bounds on neutrino interactions resulting in similar numbers.

As another example, we consider the case of a top-philic $\mathrm{ALP}$ at $\Lambda=10 \mathrm{TeV}$, with Lagrangian

$\mathcal{L}=\mathcal{L}_{\mathrm{SM}}+\frac{1}{2} \partial_{\mu} s \partial^{\mu} s+\frac{1}{2} \tilde{m}^{2} s^{2}+a_{t} s\left[i \bar{q}_{L} \tilde{\phi} t_{R}+\right.$ h.c. $]$,

where $q_{L}$ stands for the third generation quark doublet and, in our notation, $a_{t}=\left(a_{s u \phi}\right)^{33}$. As before, $a_{t}$ generates, via renormalization mixing, a non-vanishing $a_{\text {se }}$. Proceeding in the same way as above, we obtain $a_{t}(10 \mathrm{TeV}) \lesssim 4.3 \times$ $10^{-6} \mathrm{TeV}^{-1}$ from the bound $\tilde{c}_{e}(\mu \sim \mathrm{KeV}) \lesssim 3 \times 10^{-13}$. Direct bounds on this coupling could in principle be obtained from $p p \rightarrow t \bar{t} s$, but they are likely to be very weak due to the challenging final state. Other indirect constraints on $a_{t}$ have been studied in Ref. [30] but they are again much weaker than the one we have obtained.

Other interesting phenomenological implications, like the possible impact of non-SM degrees of freedom using the generic RGEs, and in particular the mixing between operators of different dimensions and different ALP content, are left for future work.

\section{Conclusions}

In this article we have investigated the EFT for ALPs up to order $\mathcal{O}(1 / \Lambda)$ in the cutoff scale $\Lambda$. We have worked in a complete basis of EFT operators to dimension five, including both shift-preserving and shift-breaking interactions. Assuming that $\mathrm{CP}$ is conserved in the UV, we have computed, at one loop, the evolution of the CP-even effective operators under renormalization group running from arbitrarily high energies down to the EW scale. In the ALP LEFT, relevant at smaller energies, in which the heavy top quark and the Higgs, $Z$ and $W$ bosons are no longer dynamical, we have also computed the renormalization of all, relevant and marginal, parameters. We have found that, in general, effective interactions can renormalize dimension-four ones, and operators involving the ALP mix with pure SM operators; although the latter effect vanishes if the theory above the EW scale involves only the ALP and SM degrees of freedom. For this latter case we have also provided the matching conditions between the EFTs above and below the EW threshold. Interestingly, we have shown that in the presence of SM dimension-five interactions below the EW scale, the ALP coupling to photons no longer renormalizes proportionally to itself.

To make our work more useful, we have not only given the full list of beta functions in a minimal basis but we have also explicitly written the corresponding counterterms of all independent (off-shell) Green functions. This is important for two reasons: first because without this information, the RGEs of extensions of our EFT, e.g. by adding right-handed neutrinos, can not be built on our results, as redundancies are different in different EFTs;

second because while in analytical one-loop computations within $\overline{\mathrm{MS}}$ the counterterms might be in principle ignored by just dropping the $1 / \epsilon$ poles, their precise value is of fundamental importance in numerical Monte Carlo simulations.

For the ALP EFT in the unbroken phase, several RGEs have been obtained previously in the literature. These assumed however shift invariance, and were therefore presented in a different set of operators, in which the ALP comes always in derivatives. We have discussed some redundancies that appear in this set of operators, and obtained conditions on the corresponding coefficients under which they actually form a basis of independent interactions. Upon relating this basis to ours, we have obtained the RGEs in the former. In this way, we have not only completely solved the full dimensionfive ALP EFT to leading-log order, but also cross-checked several of the partial results which were somewhat spread over different references $[24,59,60]$.

Finally, as an example of the utilization of our results, we have explored the possibility of indirectly probing ALP-Z as well as ALP-top interactions through their contribution to the ALP-electron coupling, which is bounded by very lowenergy measurements. (We leave the interesting possibility 
of testing ALP interactions through its mixing into pure SM ones, and vice-versa, to future work.) This shows the potential of our results to study the ALP EFT phenomenology to leading-log accuracy across all energy scales.

Acknowledgements We would like to thank Adrián Carmona for useful feedback and for suggesting the title. MC thanks also Sara Khatibi for useful discussions. MC and JS are partially supported by the Ministry of Science and Innovation under grant number FPA2016-78220C3-1/3-P (FEDER), SRA under grant PID2019-106087GB-C21/C22 (10.13039/501100011033), and by the Junta de Andalucía grants FQM 101, and A-FQM-211-UGR18 and P18-FR-4314 (FEDER). MC is also supported by the Spanish MINECO under the Ramón y Cajal programme. GG and MR acknowledge support by LIP (FCT, COMPETE2020-Portugal2020, FEDER, POCI-01-0145-FEDER-0073 34) as well as by FCT under project CERN/FIS-PAR/0024/2019. GG is also supported by FCT under the Grant SFRH/BD/144244/2019. MR is also supported by Fundação para a Ciência e Tecnologia (FCT) under the Grant PD/BD/142773/2018.

Data Availability Statement This manuscript has no associated data or the data will not be deposited. [Authors' comment: There is no data associated with this work.]

Open Access This article is licensed under a Creative Commons Attribution 4.0 International License, which permits use, sharing, adaptation, distribution and reproduction in any medium or format, as long as you give appropriate credit to the original author(s) and the source, provide a link to the Creative Commons licence, and indicate if changes were made. The images or other third party material in this article are included in the article's Creative Commons licence, unless indicated otherwise in a credit line to the material. If material is not included in the article's Creative Commons licence and your intended use is not permitted by statutory regulation or exceeds the permitted use, you will need to obtain permission directly from the copyright holder. To view a copy of this licence, visit http://creativecomm ons.org/licenses/by/4.0/.

Funded by SCOAP ${ }^{3}$.
Appendix A: One-loop diagrams in the ALP SMEFT

See Figs. 1, 2, 3, 4, 5, 6, 7, 8, 9, 10 and 11. 

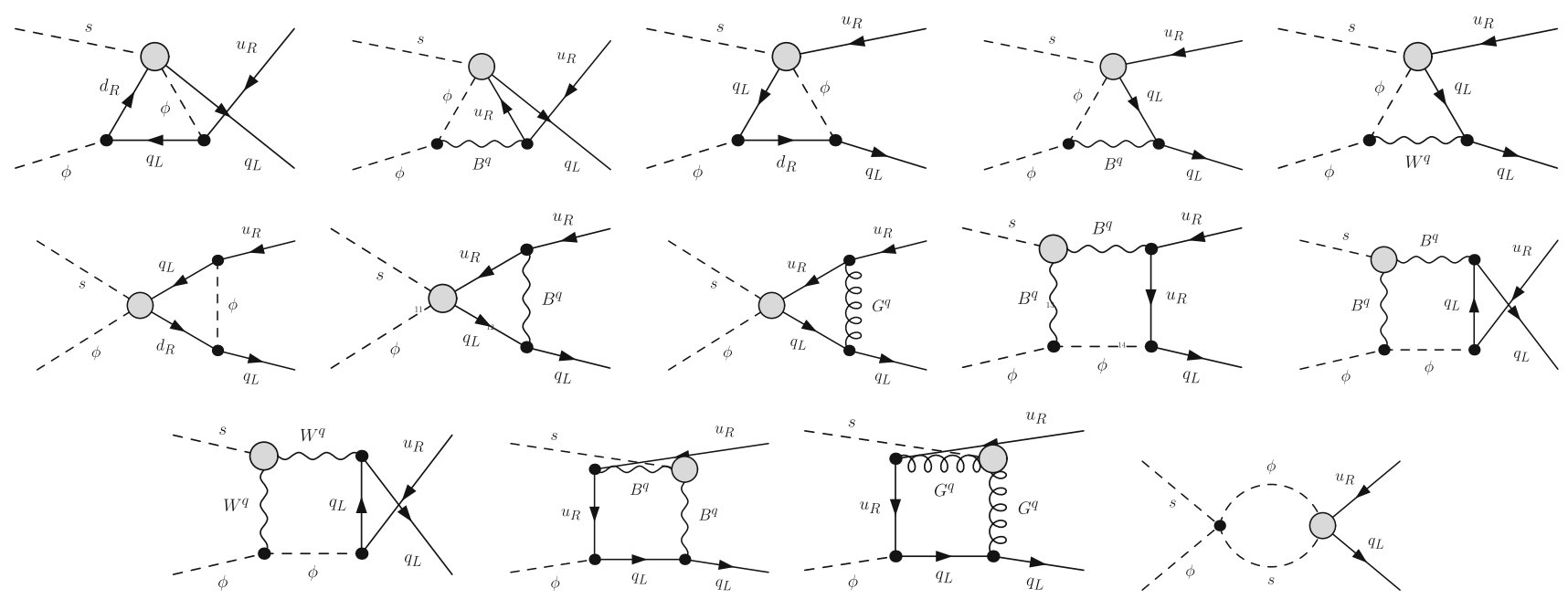

Fig. 1 Feynman diagrams for $s\left(p_{1}\right) \phi^{\dagger}\left(p_{2}\right) \rightarrow q_{L}\left(p_{3}\right) \overline{u_{R}}\left(p_{4}\right)$
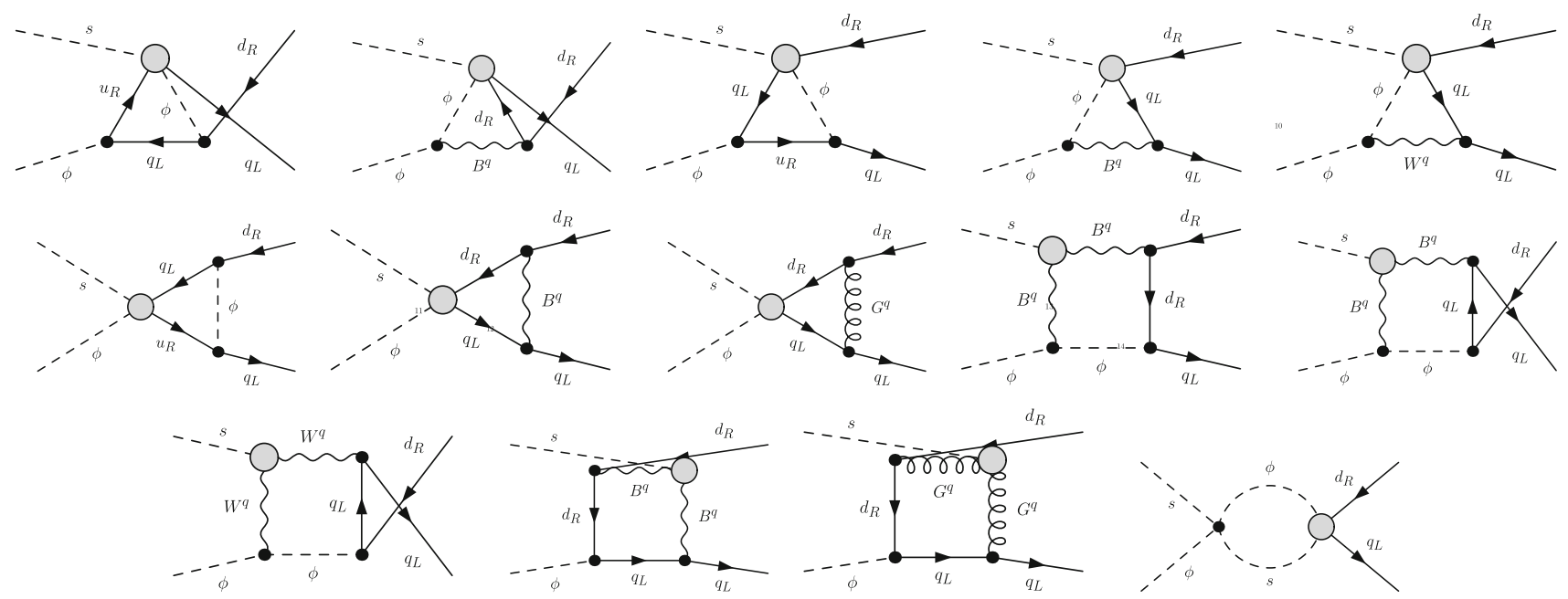

Fig. 2 Feynman diagrams for $s\left(p_{1}\right) \phi\left(p_{2}\right) \rightarrow q_{L}\left(p_{3}\right) \overline{d_{R}}\left(p_{4}\right)$
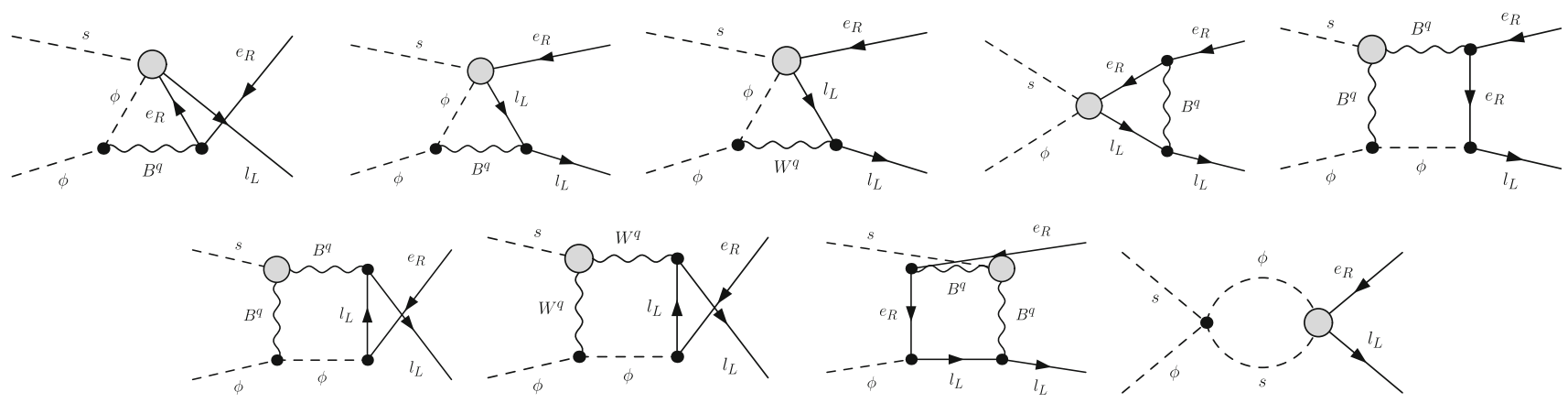

Fig. 3 Feynman diagrams for $s\left(p_{1}\right) \phi\left(p_{2}\right) \rightarrow l_{L}\left(p_{3}\right) \overline{e_{R}}\left(p_{4}\right)$ 

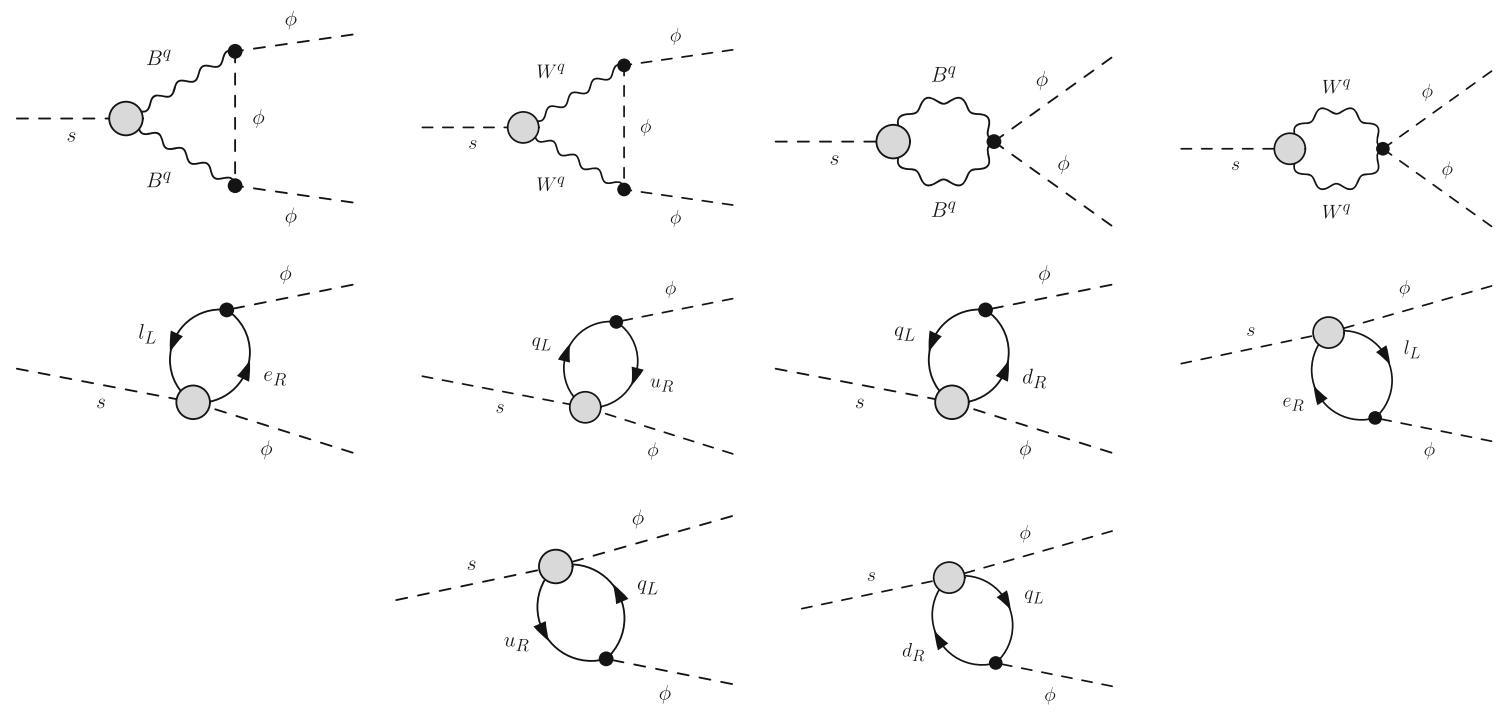

Fig. 4 Feynman diagrams for $s\left(p_{1}\right) \rightarrow \phi\left(p_{2}\right) \phi^{\dagger}\left(p_{3}\right)$
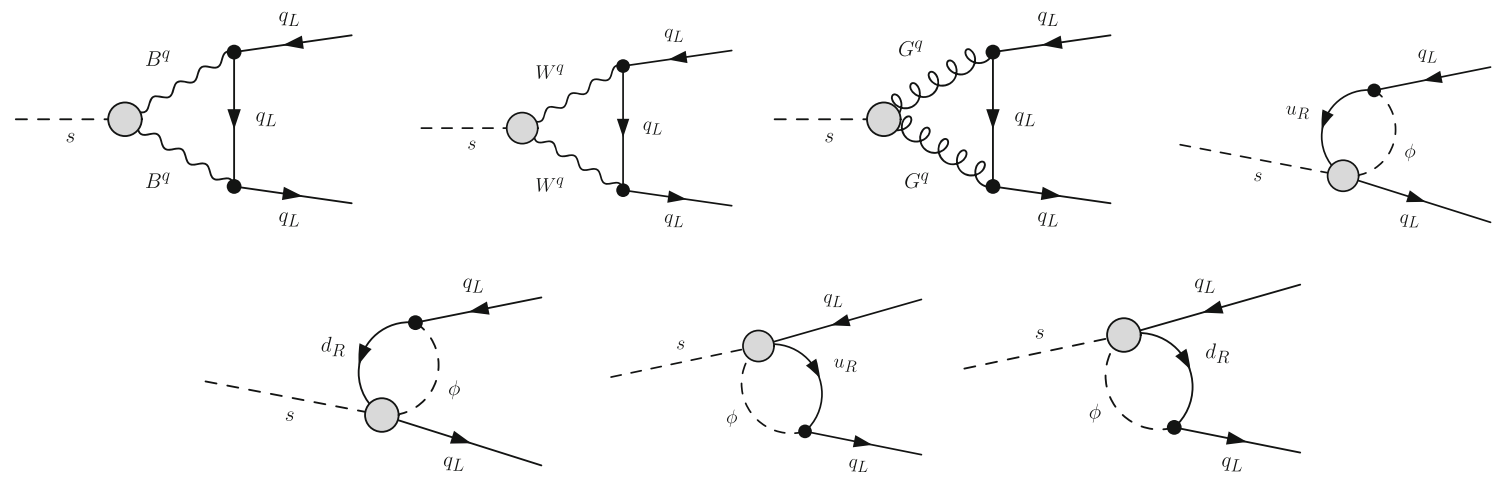

Fig. 5 Feynman diagrams for $s\left(p_{1}\right) \rightarrow q_{L}\left(p_{2}\right) \overline{q_{L}}\left(p_{3}\right)$
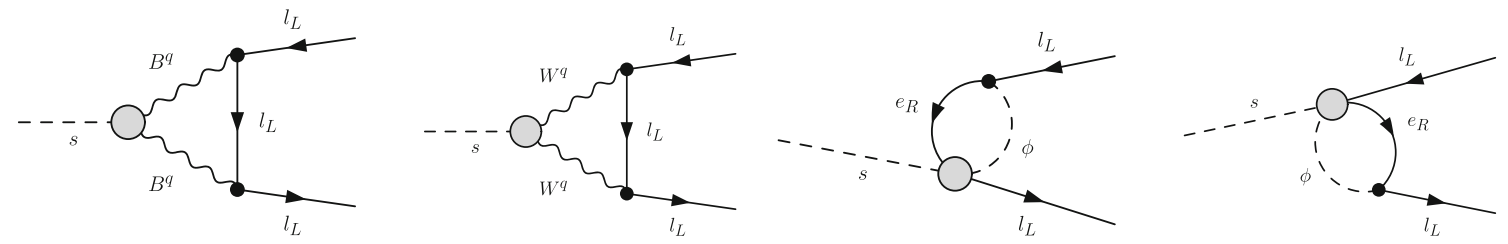

Fig. 6 Feynman diagrams for $s\left(p_{1}\right) \rightarrow l_{L}\left(p_{2}\right) \overline{l_{L}}\left(p_{3}\right)$
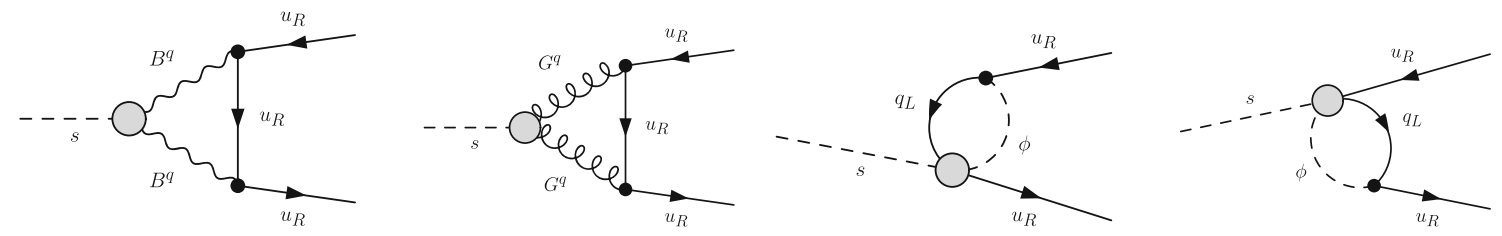

Fig. 7 Feynman diagrams for $s\left(p_{1}\right) \rightarrow u_{R}\left(p_{2}\right) \overline{u_{R}}\left(p_{3}\right)$ 

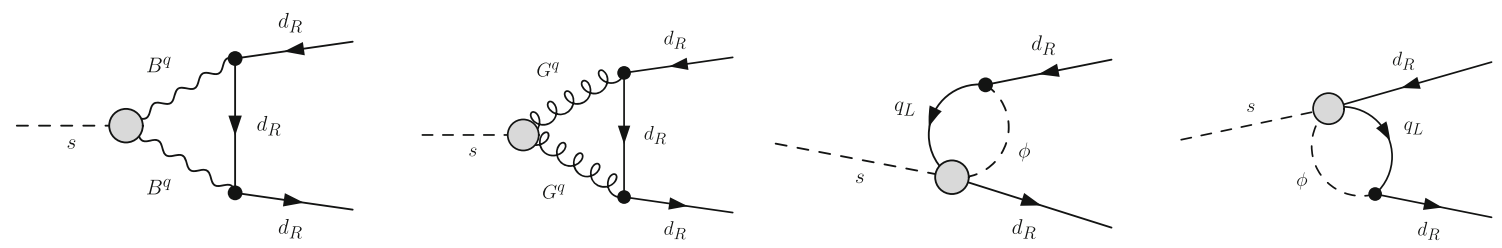

Fig. 8 Feynman diagrams for $s\left(p_{1}\right) \rightarrow d_{R}\left(p_{2}\right) \overline{d_{R}}\left(p_{3}\right)$
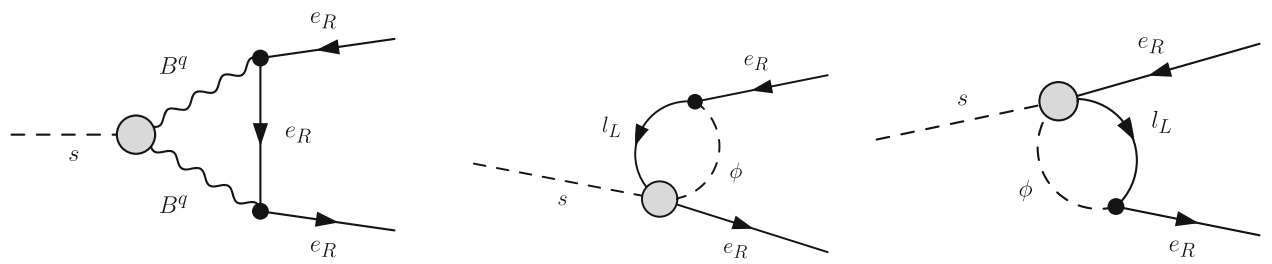

Fig. 9 Feynman diagrams for $s\left(p_{1}\right) \rightarrow e_{R}\left(p_{2}\right) \overline{e_{R}}\left(p_{3}\right)$
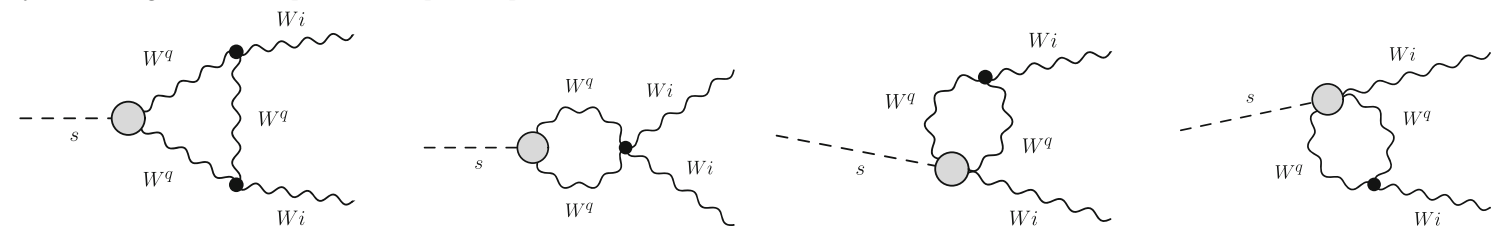

Fig. 10 Feynman diagrams for $s\left(p_{1}\right) \rightarrow W^{3}\left(p_{2}\right) W^{3}\left(p_{3}\right)$
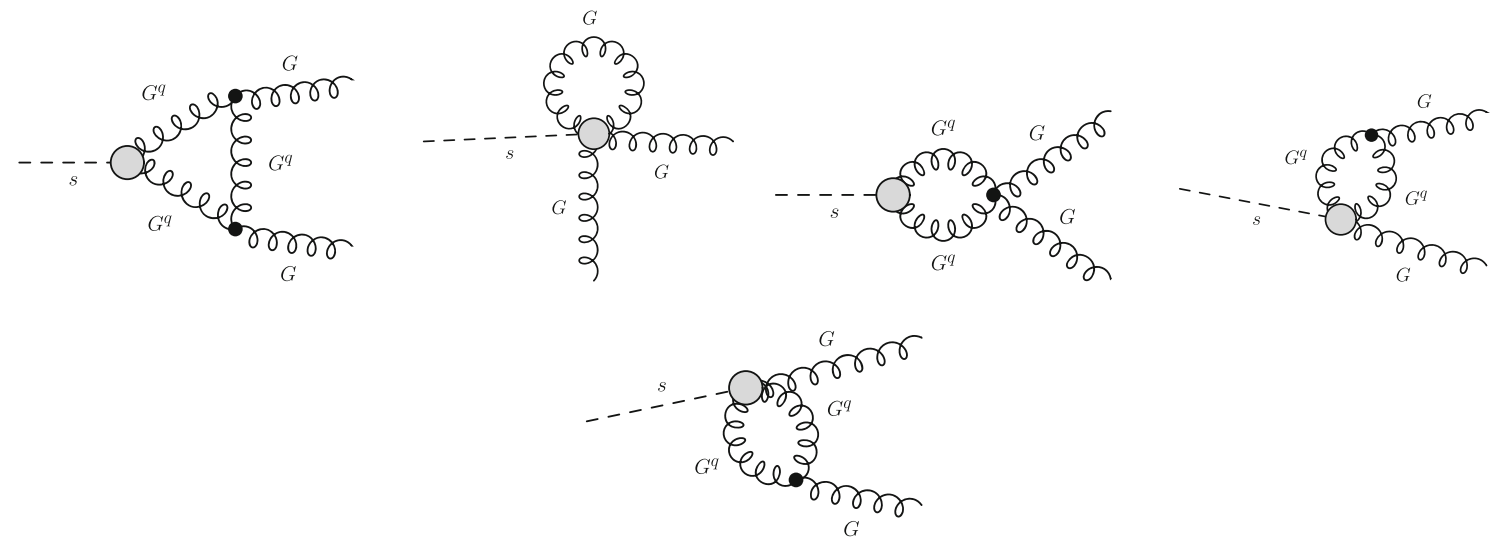

Fig. 11 Feynman diagrams for $s\left(p_{1}\right) \rightarrow G\left(p_{2}\right) G\left(p_{3}\right)$ 
Appendix B: Shift symmetry and relations between different bases

It is often interesting to assume that the ALP EFT is strictly invariant under the shift $s \rightarrow s+\sigma$, with $\sigma$ being constant. In this case, the following Lagrangian is more commonly used in the literature:

$$
\begin{aligned}
\mathcal{L}_{\text {dim-5 }}= & \sum_{\Psi}\left(\partial_{\mu} s\right) \bar{\Psi} C_{\Psi} \gamma^{\mu} \Psi+C_{B} g_{1}^{2} s B_{\mu \nu} \widetilde{B}^{\mu \nu} \\
& +C_{W} g_{2}^{2} s W_{\mu \nu}^{a} \widetilde{W}_{a}^{\mu \nu}+C_{G} g_{3}^{2} s G_{\mu \nu}^{A} \widetilde{G}_{A}^{\mu \nu},
\end{aligned}
$$

where $\Psi$ runs over $q_{L}, l_{L}, u_{R}, d_{R}, e_{R}$ and $C_{\Psi}$ are hermitian matrices in flavour space. The advantage of this parametrisation is that the shift symmetry is explicit. However, it must be noted that not all terms in the Lagrangian are independent. Indeed, focusing for concreteness on the leptons, we note that there are $9+9=18$ independent real parameters from $C_{e}$ and $C_{l}$. This is the same number of parameters that we have in the lepton sector of the (non-redundant) Lagrangian used in Sect. 2, but the latter involves all possible terms, including those that are not shift-invariant.

One could be tempted to think that the LH operators can be traded, on-shell, by the RH ones. This is however not true. In general, the following relation holds on shell:

$\partial_{\mu} s \overline{l_{L}} C_{l} \gamma^{\mu} l_{L}=\partial_{\mu} s \overline{e_{R}} H \gamma^{\mu} e_{R}+s \overline{e_{R}}(\mathrm{i} A) \mathrm{i} \overleftrightarrow{\not D} e_{R}$,

where $\overleftrightarrow{D_{\mu}} \equiv D_{\mu}-\overleftarrow{D_{\mu}}$ and $H$ and $A$ are hermitian and anti-hermitian matrices, respectively, given by

$$
\begin{aligned}
A & =\frac{1}{2}\left[\left(y^{e}\right)^{-1} C_{l} y^{e}-y^{e^{\dagger}} C_{l}\left(y^{e}\right)^{-1 \dagger}\right], \\
H & =-\frac{1}{2}\left[\left(y^{e}\right)^{-1} C_{l} y^{e}+y^{e \dagger} C_{l}\left(y^{e}\right)^{-1 \dagger}\right] .
\end{aligned}
$$

So the LH and RH operators are only equivalent if $A$ vanishes. A sufficient condition for this to happen is that the Yukawa coupling commutes with $C_{l}$, which happens in particular if the ALP couples to a single lepton family.

The on-shell relation in Eq. (172) might seem counterintuitive at first, since the left-hand side is explicitly shiftinvariant but the term in the right-hand side proportional to $A$ does not seem to be. However a closer inspection shows that this term is in fact shift-invariant as it should. To prove it, let us consider the operator

$s \overline{e_{R}} C \mathrm{i} \overleftrightarrow{\not D} e_{R}$

with $C$ being an arbitrary hermitian matrix of order $1 / \Lambda$. In order to see under which conditions this operator is shift invariant we perform a shift, $s \rightarrow s+\sigma$,

$s \overline{e_{R}} C \mathrm{i} \overleftrightarrow{\not D} e_{R} \rightarrow(s+\sigma) \overline{e_{R}} C \mathrm{i} \overleftrightarrow{\not D} e_{R}$ and we get an extra contribution to the $e$ kinetic term, which can be canonically normalized via the following field redefinition,

$e_{R} \rightarrow(1-\sigma C) e_{R}$

Since $C$ is of order $1 / \Lambda$, the only effect of this field redefinition happens in the renormalizable Lagrangian and therefore only in the Yukawa term:

$\overline{l_{L}} \phi y^{e} e \rightarrow \overline{l_{L}} \phi\left(y^{e}-\sigma y^{e} C\right) e_{R}$.

We can now perform arbitrary chiral rotations which, to the order we are considering, read

$l_{L} \rightarrow\left(1+A_{L}\right) l_{L}, \quad e_{R} \rightarrow\left(1+A_{R}\right) e_{R}$,

where $A_{L, R}$ are anti-hermitian matrices of order $\sigma / \Lambda$. Under these rotations, the Yukawa Lagrangian receives the following correction:

$\overline{l_{L}} \phi y^{e} e_{R} \rightarrow \overline{l_{L}} \phi\left(y^{e}-\sigma y^{e} C-A_{L} y^{e}+y^{e} A_{R}\right) e_{R}$,

which is independent of $\sigma$ (and therefore shift invariant) if

$y^{e} C=\frac{y^{e} A_{R}-A_{L} y^{e}}{\sigma}$.

Using the hermiticity of $C$ we can eliminate $A_{R}$ and get the following sufficient condition for shift invariance:

$C^{\text {shift-inv }}=y^{e \dagger} A_{L}\left(y^{e \dagger}\right)^{-1}-\left(y^{e}\right)^{-1} A_{L} y^{e}$,

with $A_{L}$ being an arbitrary anti-hermitian matrix of order $1 / \Lambda$ (we have absorbed in its definition a factor of $1 / 2 \sigma$ ). We see from Eq. (173) that i $A$ has precisely that form with the identification $A_{L}=-\mathrm{i} C_{l} / 2$ and therefore Eq. (172) is, as anticipated, shift invariant.

Equation (172) shows that the Lagrangian in Eq. (171) is in general redundant but also that, if $A$ vanishes, a minimal basis is given by eliminating the operators with $\mathrm{LH}$ fields in this equation. In this case the Wilson coefficients are univocally related in both bases and we can translate the anomalous dimensions to the new one. The relations between the Wilson coefficients are:

$$
\begin{aligned}
a_{s B} & =C_{B} g_{1}^{2}, \\
a_{s W} & =C_{W} g_{2}^{2}, \\
a_{s G} & =C_{G} g_{3}^{2}, \\
a_{s \psi \phi} & =-\operatorname{Re}\left(y^{\psi} C_{\psi_{R}}\right), \\
a_{\widetilde{s \psi \phi}} & =\operatorname{Im}\left(y^{\psi} C_{\psi_{R}}\right),
\end{aligned}
$$

where $\psi$ stands for $u, d$ or $e$. Using the results in Sect. 4 and the SM RGEs in Appendix C, we can obtain the RGEs in this new basis, valid in the limit of vanishing complex phases for the Yukawa couplings. The results are presented in Eqs. (61)-(63). 
Note that the ALP-fermion-fermion operators in the new basis are not renormalized by gauge interactions. The gauge contributions to the $s \bar{\Psi} \phi \Psi$ operators is exactly cancelled by the running of the Yukawa couplings. This result can be ultimately rooted to the fact that $\bar{\psi} \gamma_{\mu} \psi$ is a conserved current of $U(1)$ and therefore does not renormalize to itself off-shell [76], and by noticing that the non-abelian structure of the EW and color gauge interactions does not manifest in the renormalization of $\partial_{\mu} s \bar{\psi} \gamma_{\mu} \psi$ at one loop.

Given the discussion so far, we find worth discussing shift invariance in the basis of operators introduced in Sect. 2, which contrary to Eq. (171) is minimal. We find the following sufficient conditions on the Wilson coefficients for a shiftinvariant theory:

$a_{s u \phi}^{\text {shift-inv }}=\operatorname{Re}\left(H_{q_{L}} y^{u}+y^{u} H_{u_{R}}\right)$,

$a_{\text {su } \phi}^{\text {shift-inv }}=-\operatorname{Im}\left(H_{q_{L}} y^{u}+y^{u} H_{u_{R}}\right)$,

$a_{s d \phi}^{\text {shift-inv }}=\operatorname{Re}\left(H_{q_{L}} y^{d}+y^{d} H_{d_{R}}\right)$

$a_{\text {sd } \phi}^{\text {shift-inv }}=-\operatorname{Im}\left(H_{q_{L}} y^{d}+y^{d} H_{d_{R}}\right)$,

$a_{\text {se }}^{\text {shift-inv }}=\operatorname{Re}\left(H_{l_{L}} y^{e}+y^{e} H_{e_{R}}\right)$,

$a \widetilde{\text { shift}}^{\text {shv }}=-\operatorname{Im}\left(H_{l_{L}} y^{e}+y^{e} H_{e_{R}}\right)$,

with $H_{q_{L}, l_{L}, u_{R}, d_{R}, e_{R}}$ being arbitrary hermitian matrices.

Let us see that this is indeed the case. We show it explicitly for the case of leptons, quarks being a straightforward generalization. Let us consider the following Lagrangian:

$\mathcal{L}=-\overline{l_{L}}\left(y^{e}-\mathrm{i} s \alpha_{e}\right) \phi e_{R}+$ h.c. $+\cdots$,

where $\alpha_{e}$ is an arbitrary matrix in flavour space of order $1 / \Lambda$ and the dots stand for other interactions that are not relevant for the present discussion. A shift $s \rightarrow s+\sigma$ induces the following change in the Lagrangian:

$\mathcal{L} \rightarrow \mathcal{L}-\left[\overline{l_{L}}\left(-\mathrm{i} \sigma \alpha_{e}\right) \phi e_{R}+\right.$ h.c. $]$.

Performing again arbitrary chiral rotations as in Eq. (179) we get, to the order we are considering,

$\mathcal{L} \rightarrow \mathcal{L}-\left[\overline{l_{L}}\left(y^{e} A_{e_{R}}-A_{l_{L}} y^{e}-\mathrm{i} \sigma \alpha_{e}\right) \phi e_{R}+\right.$ h.c. $]$.

We therefore see that a sufficient condition for $\alpha_{e}$ to keep shift invariance is that it has the form

$\alpha_{e}^{\text {shift-inv }}=H_{l_{L}} y^{e}+y^{e} H_{e_{R}}$,

with $H_{l_{L}, e_{R}}$ being arbitrary hermitian matrices, corresponding to $\pm \mathrm{i} A_{l_{L}, e_{R}} / \sigma$ in Eq. (196), respectively. Taking now into account the relation between $\alpha_{e}$ and the Wilson coefficients in our basis, namely,

$a_{s e \phi}=\operatorname{Re} \alpha_{e}, \quad a_{s e \phi}=-\operatorname{Im} \alpha_{e}$,

we obtain the condition in Eqs. (188)-(193).
Appendix C: Running of renormalizable parameters above the electroweak scale

The renormalizable parameters of the theory above EWSB evolve following:

$$
\begin{aligned}
\beta_{g_{1}}= & \frac{41}{6} g_{1}^{3}, \\
\beta_{g_{2}}= & -\frac{19}{6} g_{2}^{3}, \\
\beta_{g_{3}=}= & -7 g_{3}^{3}, \\
\beta_{m^{2}}= & 4 \lambda_{s \phi} \mu^{2}+\lambda_{s} m^{2} \\
\beta_{\mu^{2}=} & \lambda_{s \phi} m^{2}+\left[2 \operatorname{Tr}\left(y^{e} y^{e^{\dagger}}\right)\right. \\
& \left.+6 \operatorname{Tr}\left(y^{u} y^{u \dagger}\right)+6 \operatorname{Tr}\left(y^{d} y^{d^{\dagger}}\right)-\frac{3}{2} g_{1}^{2}-\frac{9}{2} g_{2}^{2}-12 \lambda\right] \mu^{2},
\end{aligned}
$$

$\beta_{\lambda_{s}}=3 \lambda_{s}^{2}+12 \lambda_{s \phi}^{2}$,$$
\beta_{\lambda_{s \phi}}=\left[\lambda_{s}+4 \lambda_{s \phi}+2 \operatorname{Tr}\left(y^{e} y^{e^{\dagger}}\right)+6 \operatorname{Tr}\left(y^{u} y^{u \dagger}\right)\right.
$$$$
\left.+6 \operatorname{Tr}\left(y^{d} y^{d^{\dagger}}\right)+12 \lambda-\frac{3}{2} g_{1}^{2}-\frac{9}{2} g_{2}^{2}\right] \lambda_{s \phi},
$$

$$
\beta_{\lambda}=\frac{1}{2} \lambda_{s \phi}^{2}-6 \operatorname{Tr}\left(y^{u} y^{u \dagger} y^{u} y^{u \dagger}\right)-6 \operatorname{Tr}\left(y^{d} y^{d^{\dagger}} y^{d} y^{d^{\dagger}}\right)
$$$$
-2 \operatorname{Tr}\left(y^{e} y^{e^{\dagger}} y^{e} y^{e \dagger}\right)+\frac{3}{8} g_{1}^{4}+\frac{9}{8} g_{2}^{4}+\frac{3}{4} g_{1}^{2} g_{2}^{2}
$$$$
+\left[24 \lambda-3 g_{1}^{2}-9 g_{2}^{2}+4 \operatorname{Tr}\left(y^{e} y^{e^{\dagger}}\right)\right.
$$$$
\left.+12 \operatorname{Tr}\left(y^{u} y^{u \dagger}\right)+12 \operatorname{Tr}\left(y^{d} y^{d^{\dagger}}\right)\right] \lambda \text {, }
$$

$$
\begin{aligned}
\beta_{y^{u}}= & \left\{\frac{3}{2} y^{u} y^{u^{\dagger}}-\frac{3}{2} y^{d} y^{d^{\dagger}}\right. \\
& +3\left[\operatorname{Tr}\left(y^{u} y^{u^{\dagger}}\right)+\operatorname{Tr}\left(y^{d} y^{d^{\dagger}}\right)\right] \\
& \left.+\operatorname{Tr}\left(y^{e} y^{e^{\dagger}}\right)-\frac{17}{12} g_{1}^{2}-\frac{9}{4} g_{2}^{2}-8 g_{3}^{2}\right\} y^{u},
\end{aligned}
$$

$$
\begin{aligned}
\beta_{y^{d}}= & \left\{\frac{3}{2} y^{d} y^{d^{\dagger}}-\frac{3}{2} y^{u} y^{u^{\dagger}}+3\left[\operatorname{Tr}\left(y^{u} y^{u^{\dagger}}\right)+\operatorname{Tr}\left(y^{d} y^{d^{\dagger}}\right)\right]\right. \\
& \left.+\operatorname{Tr}\left(y^{e} y^{e^{\dagger}}\right)-\frac{5}{12} g_{1}^{2}-\frac{9}{4} g_{2}^{2}-8 g_{3}^{2}\right\} y^{d},
\end{aligned}
$$

$$
\begin{aligned}
\beta_{y^{e}}= & \left\{\frac{3}{2} y^{e} y^{e^{\dagger}}+3\left[\operatorname{Tr}\left(y^{u} y^{u \dagger}\right)+\operatorname{Tr}\left(y^{d} y^{d^{\dagger}}\right)\right]\right. \\
& \left.+\operatorname{Tr}\left(y^{e} y^{e^{\dagger}}\right)-\frac{15}{4} g_{1}^{2}-\frac{9}{4} g_{2}^{2}\right\} y^{e} .
\end{aligned}
$$

\section{Appendix D: One-loop diagrams in the ALP LEFT}

See Figs. 12, 13, 14, 15, 16, 17, 18, 19, 20, 21, 22, 23, 24, 25, 26, 27, 28, 29, 30 and 31 . 

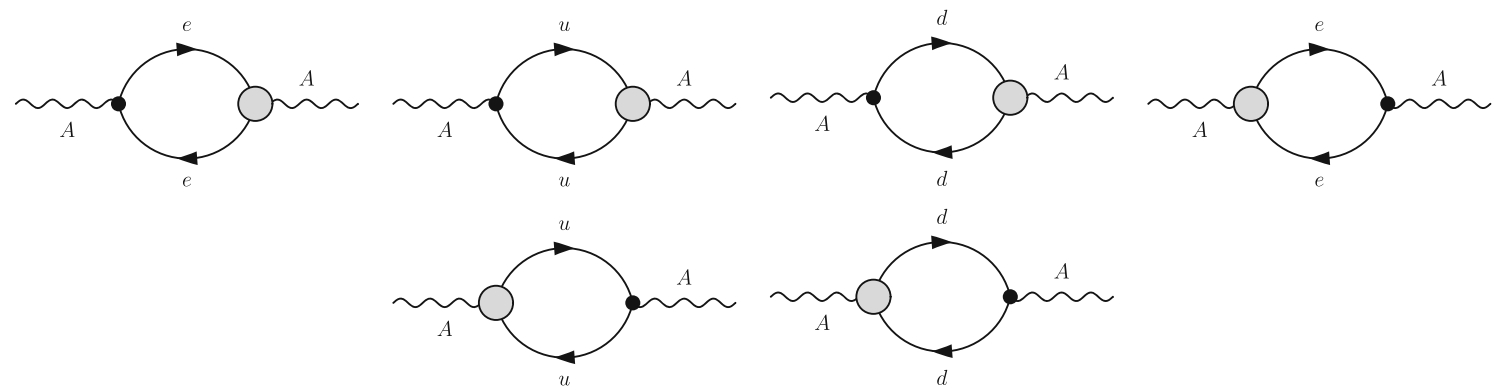

Fig. 12 Feynman diagrams for $A\left(p_{1}\right) \rightarrow A\left(p_{2}\right)$
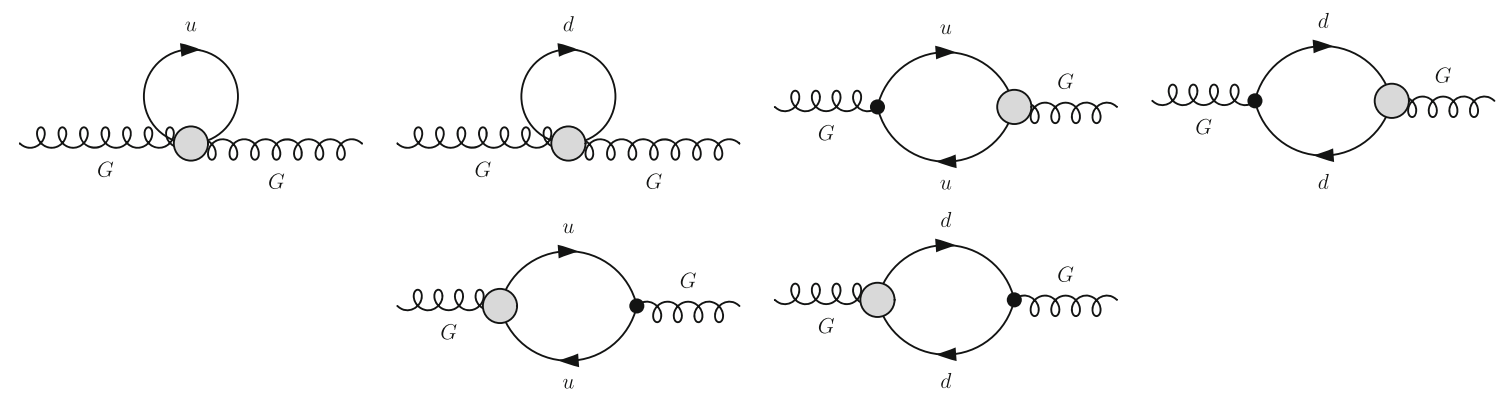

Fig. 13 Feynman diagrams for $G\left(p_{1}\right) \rightarrow G\left(p_{2}\right)$
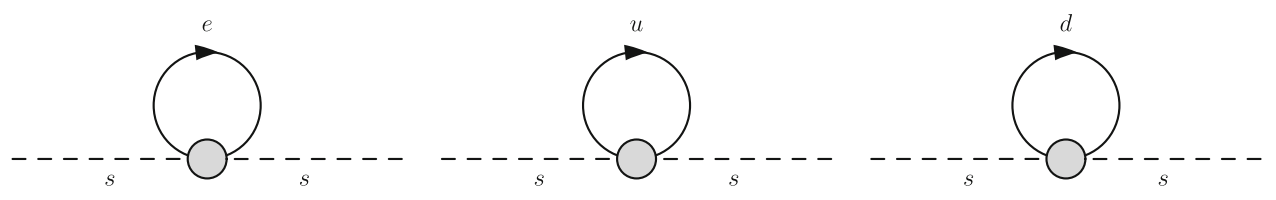

Fig. 14 Feynman diagrams for $s\left(p_{1}\right) \rightarrow s\left(p_{2}\right)$
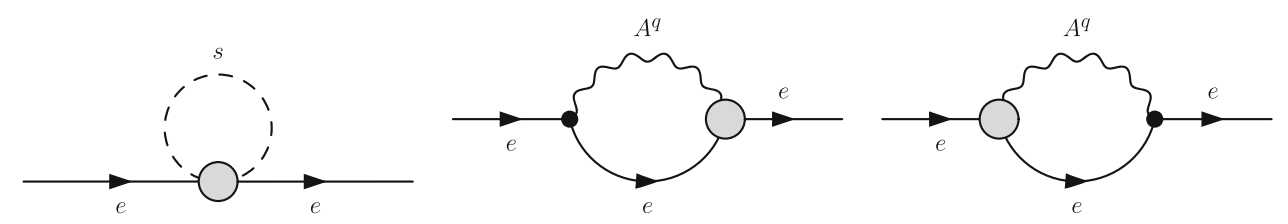

Fig. 15 Feynman diagrams for $e\left(p_{1}\right) \rightarrow e\left(p_{2}\right)$
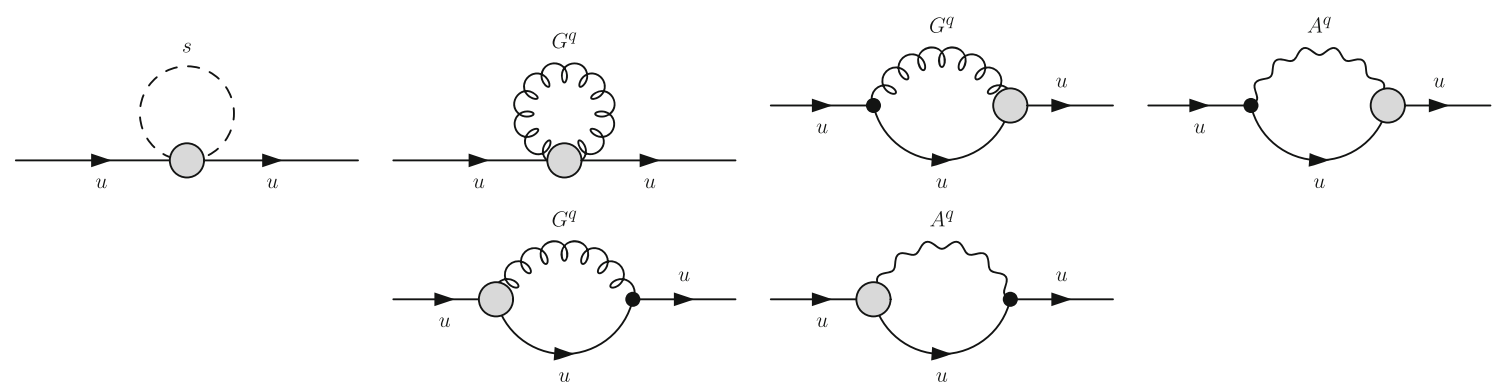

Fig. 16 Feynman diagrams for $u\left(p_{1}\right) \rightarrow u\left(p_{2}\right)$ 

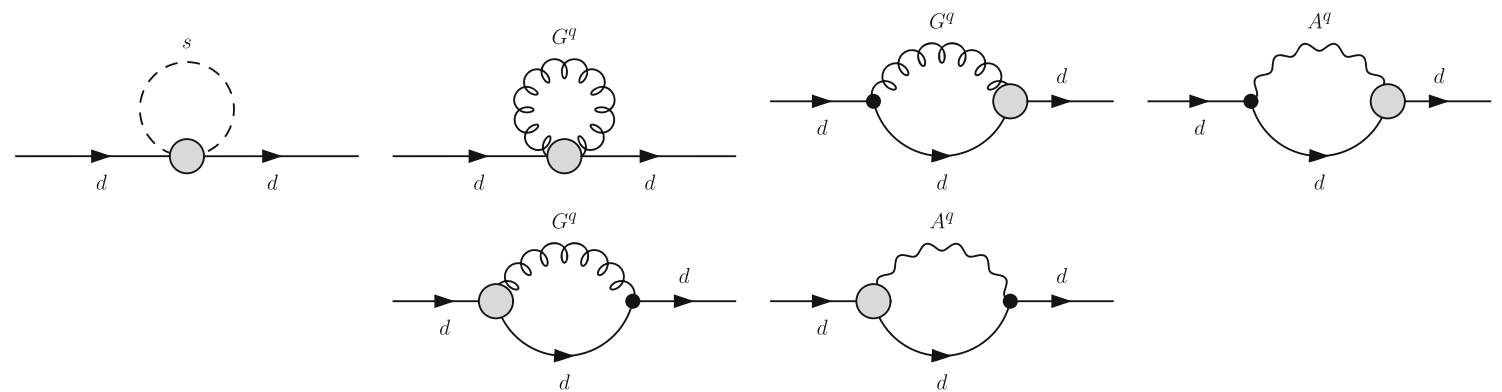

Fig. 17 Feynman diagrams for $d\left(p_{1}\right) \rightarrow d\left(p_{2}\right)$ 

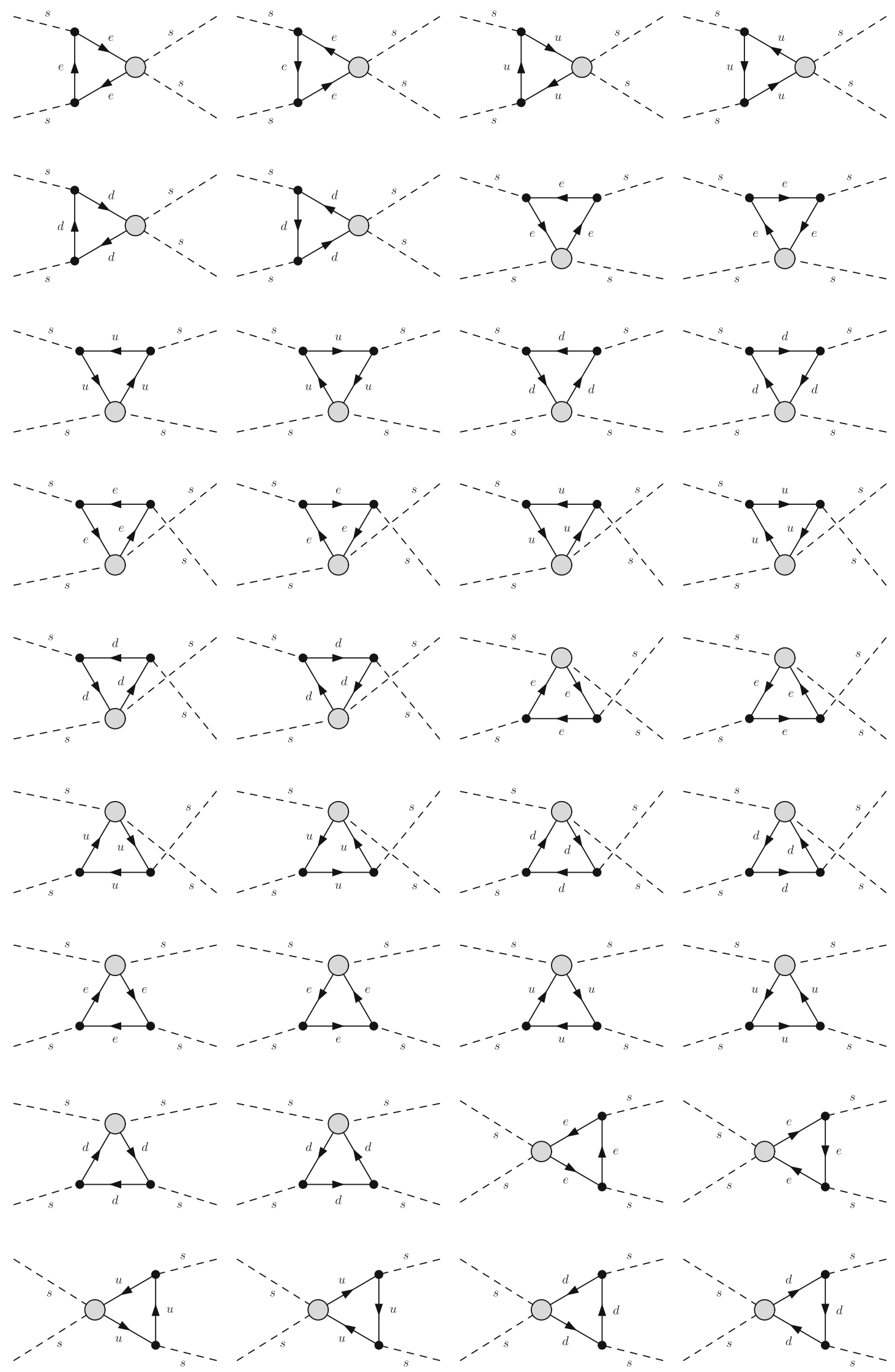

Fig. 18 Feynman diagrams for $s\left(p_{1}\right) s\left(p_{2}\right) \rightarrow s\left(p_{3}\right) s\left(p_{4}\right)$ 

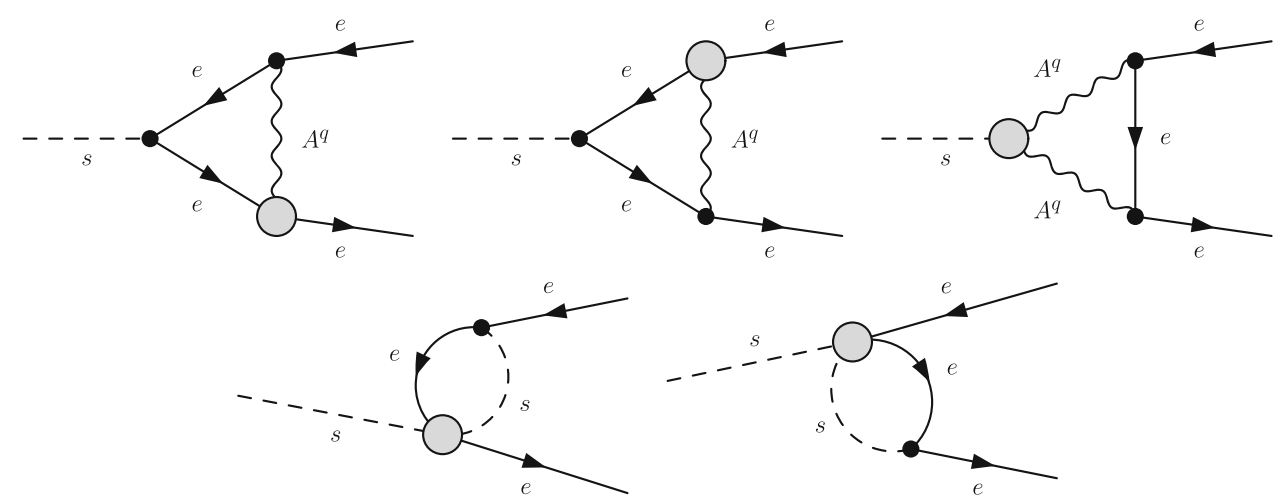

Fig. 19 Feynman diagrams for $s\left(p_{1}\right) \rightarrow \bar{e}\left(p_{2}\right) e\left(p_{3}\right)$
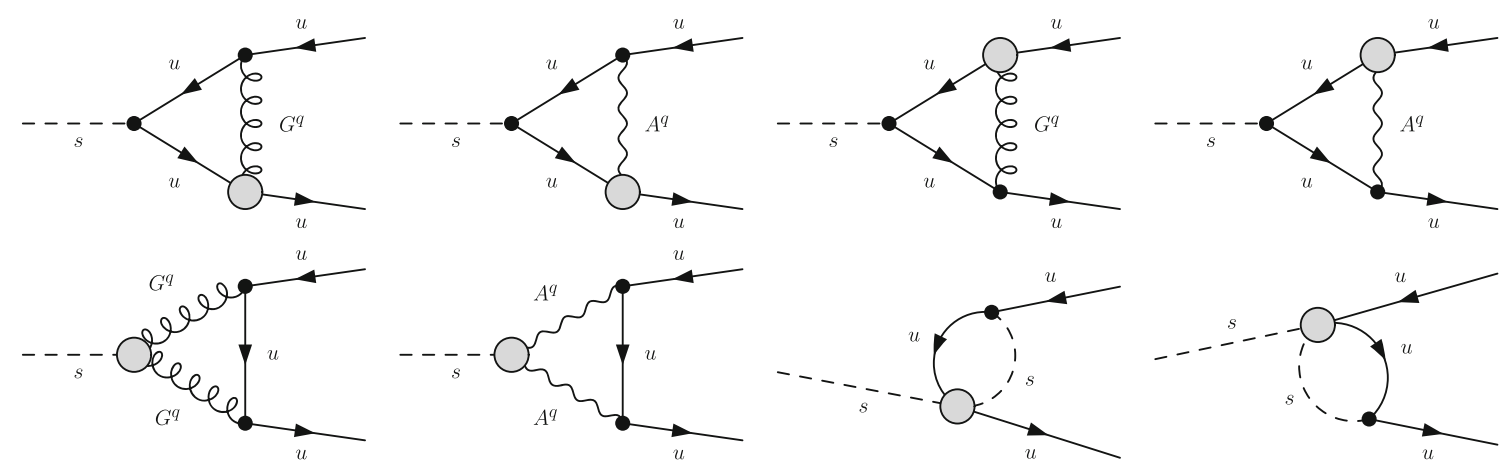

Fig. 20 Feynman diagrams for $s\left(p_{1}\right) \rightarrow \bar{u}\left(p_{2}\right) u\left(p_{3}\right)$
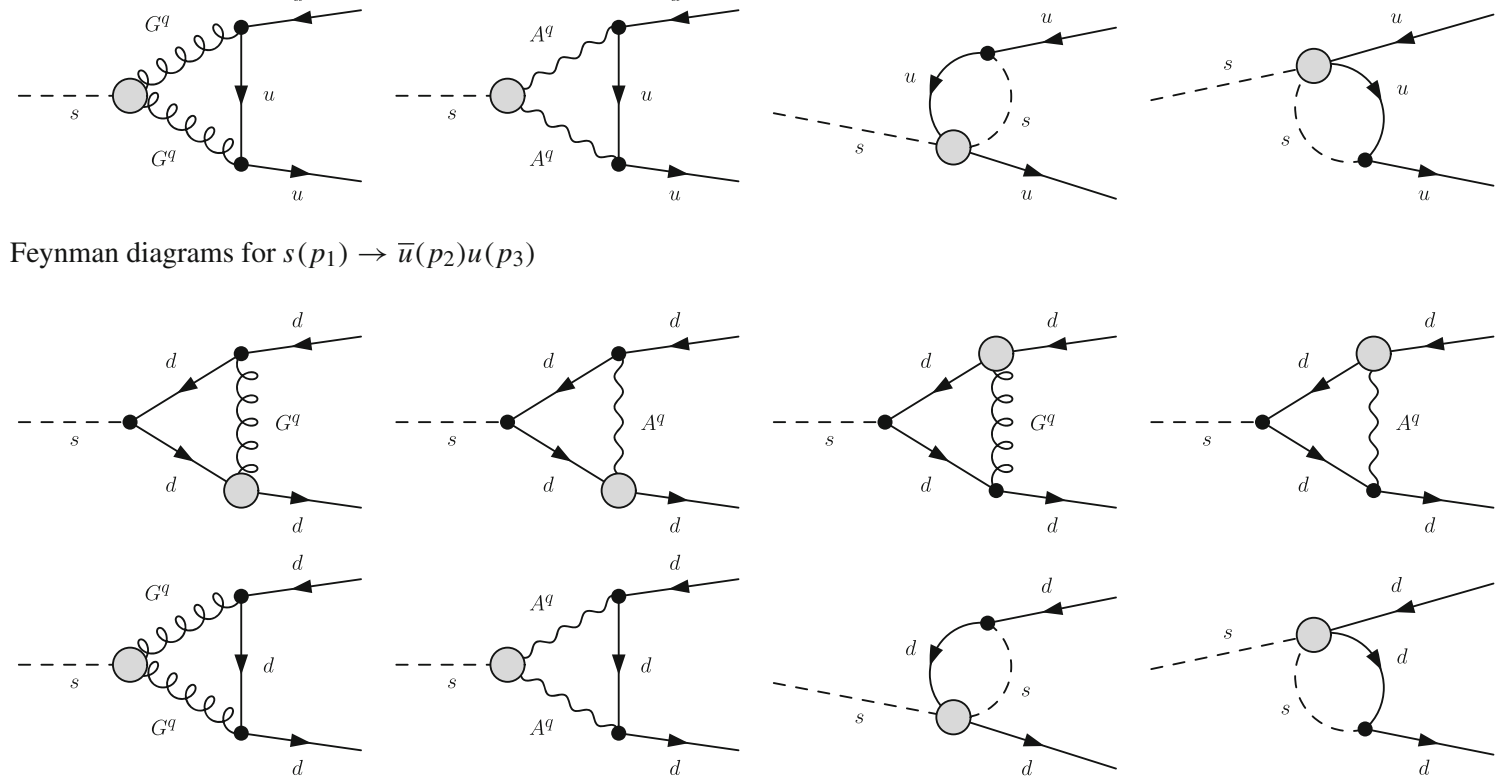

Fig. 21 Feynman diagrams for $s\left(p_{1}\right) \rightarrow \bar{d}\left(p_{2}\right) d\left(p_{3}\right)$ 

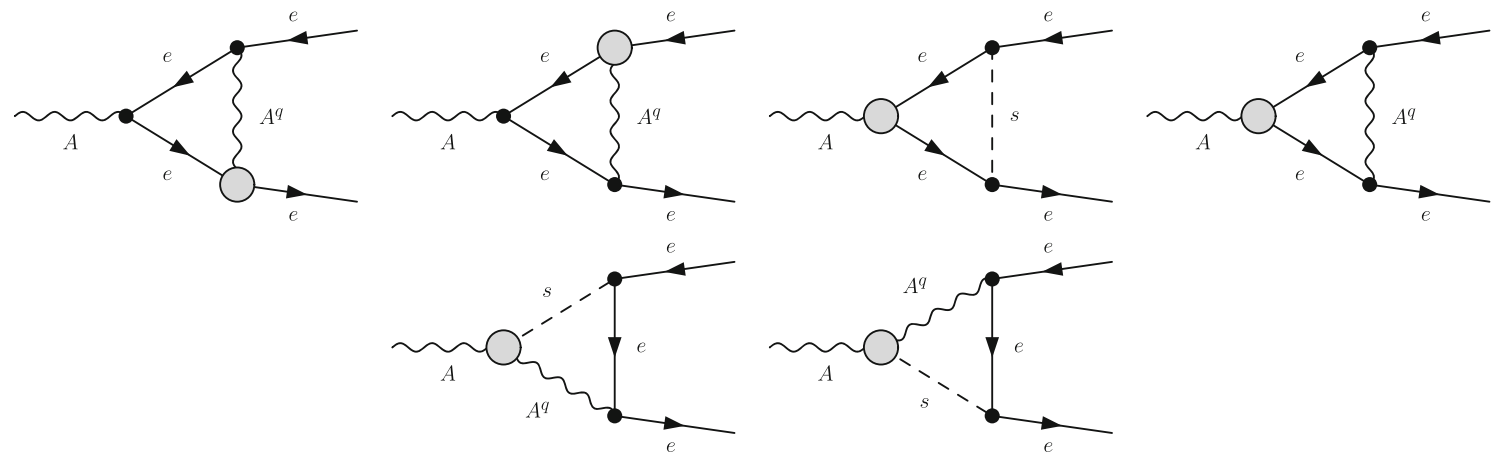

Fig. 22 Feynman diagrams for $A\left(p_{1}\right) \rightarrow \bar{e}\left(p_{2}\right) e\left(p_{3}\right)$
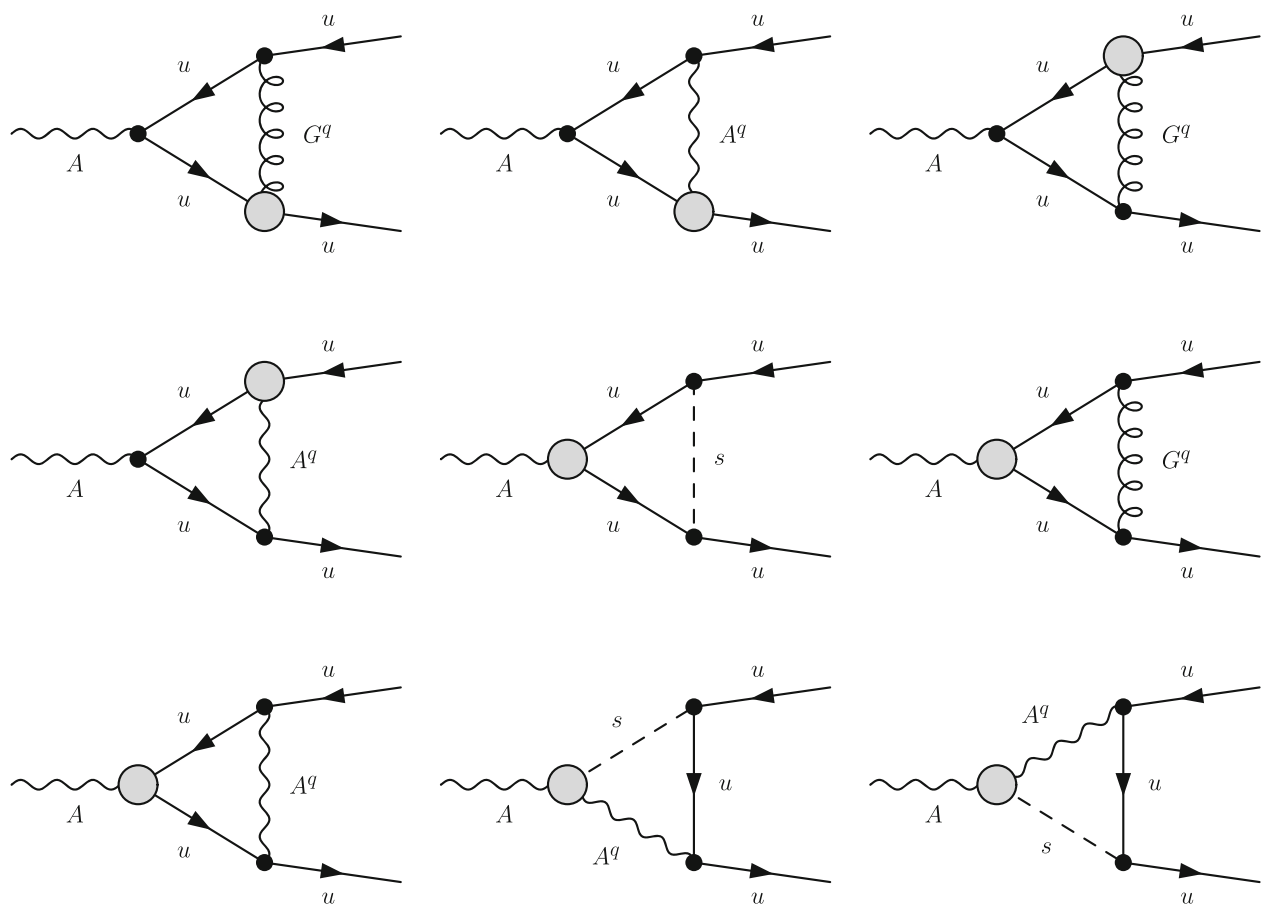

Fig. 23 Feynman diagrams for $A\left(p_{1}\right) \rightarrow \bar{u}\left(p_{2}\right) u\left(p_{3}\right)$ 

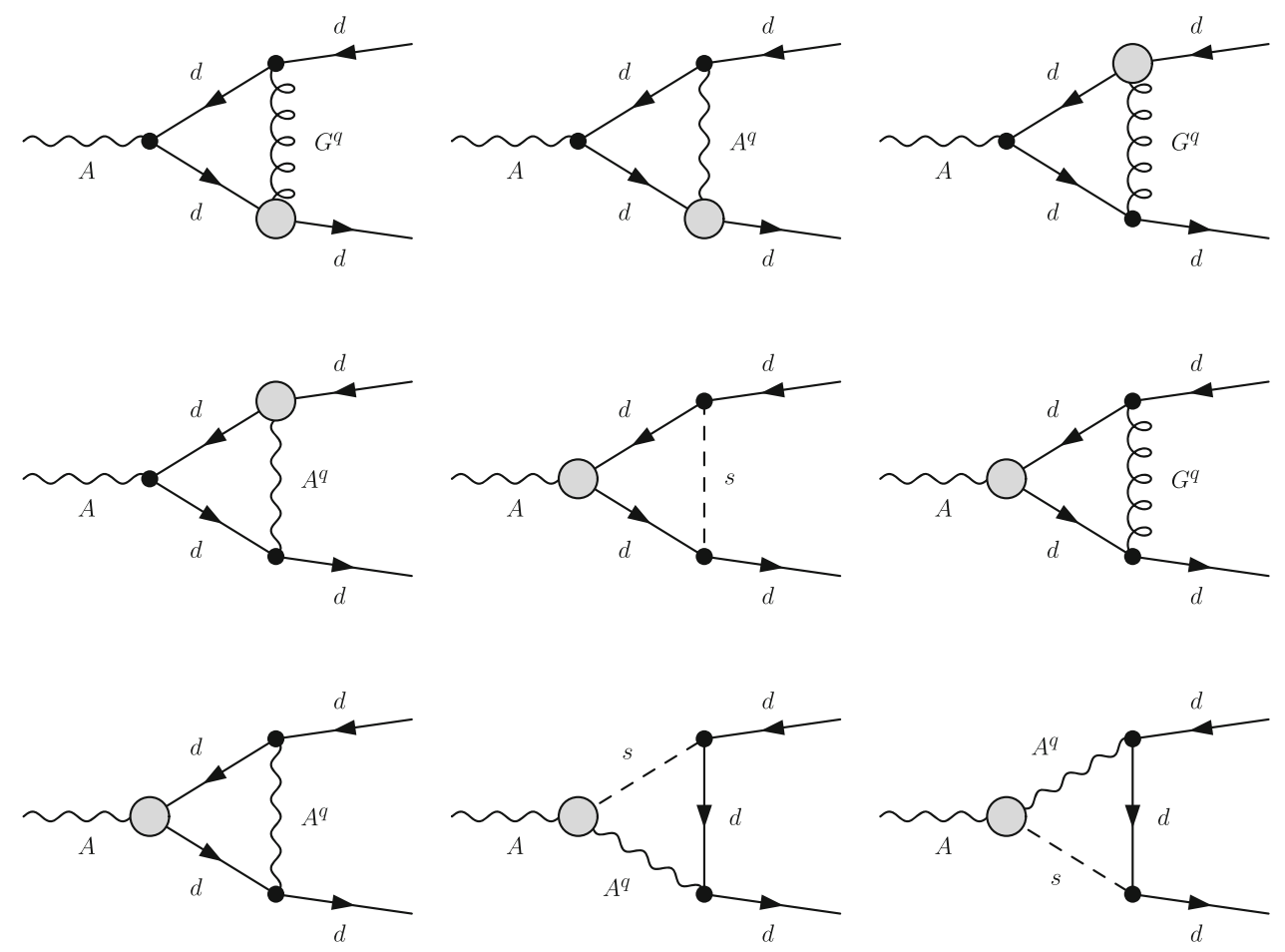

Fig. 24 Feynman diagrams for $A\left(p_{1}\right) \rightarrow \bar{d}\left(p_{2}\right) d\left(p_{3}\right)$
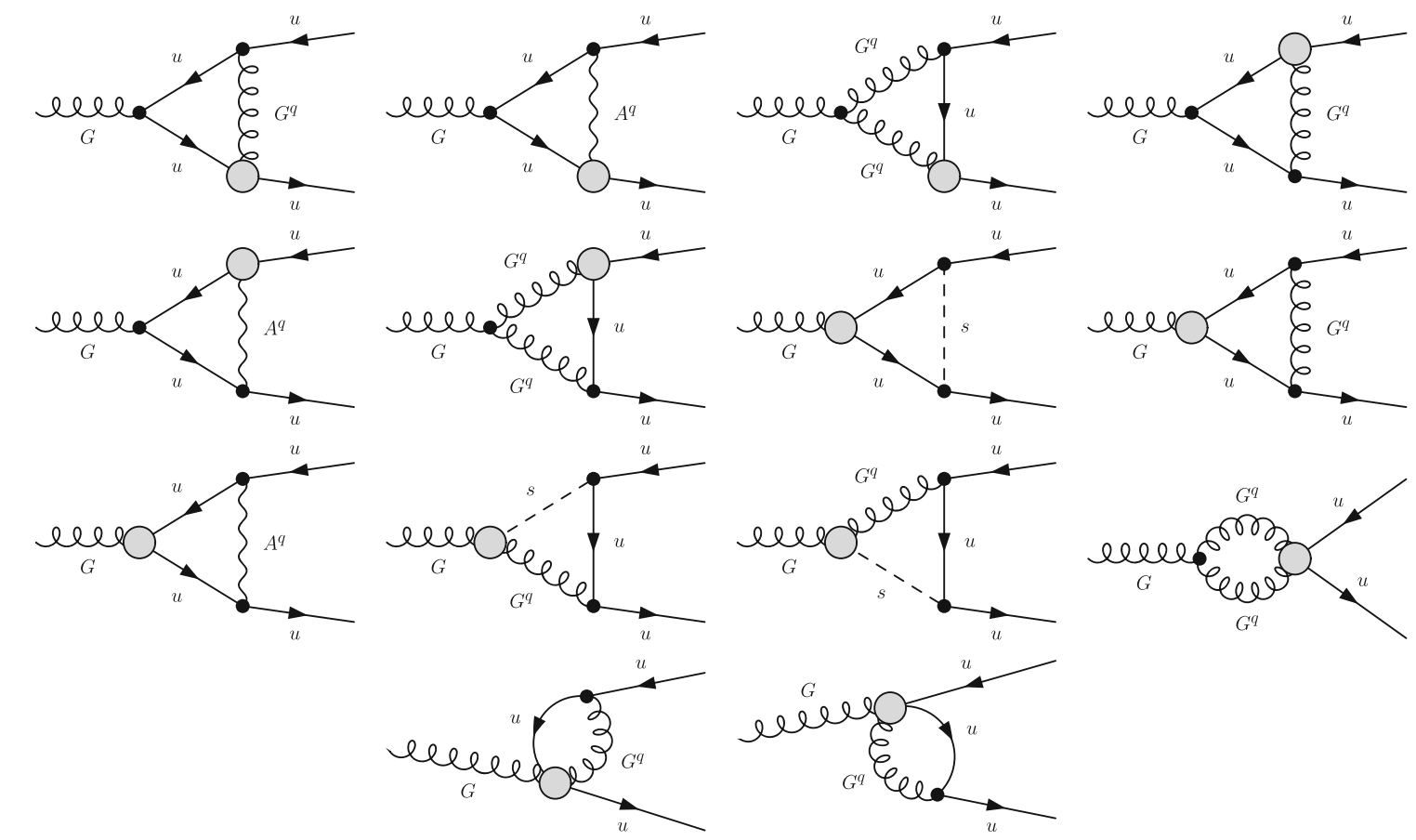

Fig. 25 Feynman diagrams for $G\left(p_{1}\right) \rightarrow \bar{u}\left(p_{2}\right) u\left(p_{3}\right)$ 

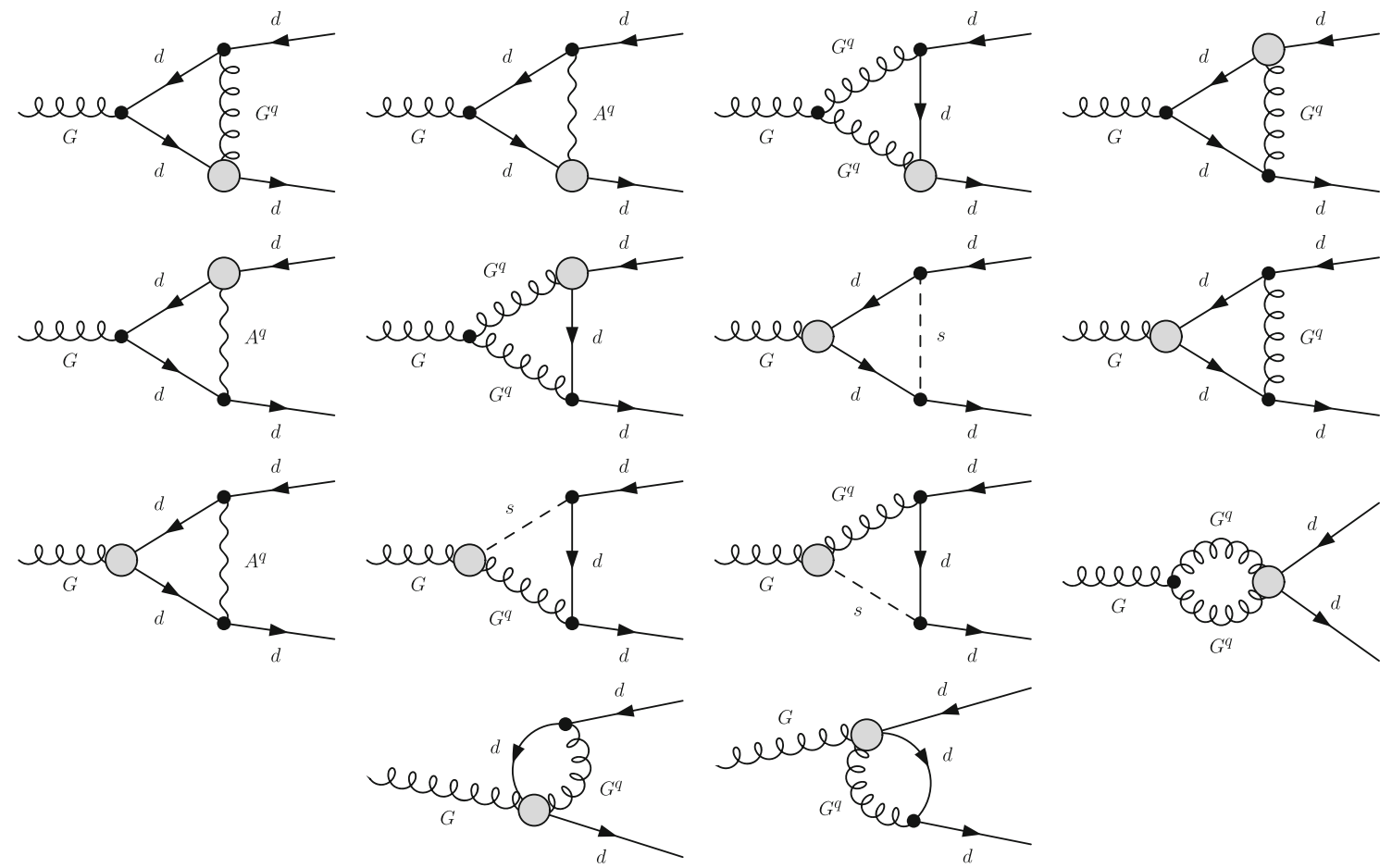

Fig. 26 Feynman diagrams for $G\left(p_{1}\right) \rightarrow \bar{d}\left(p_{2}\right) d\left(p_{3}\right)$

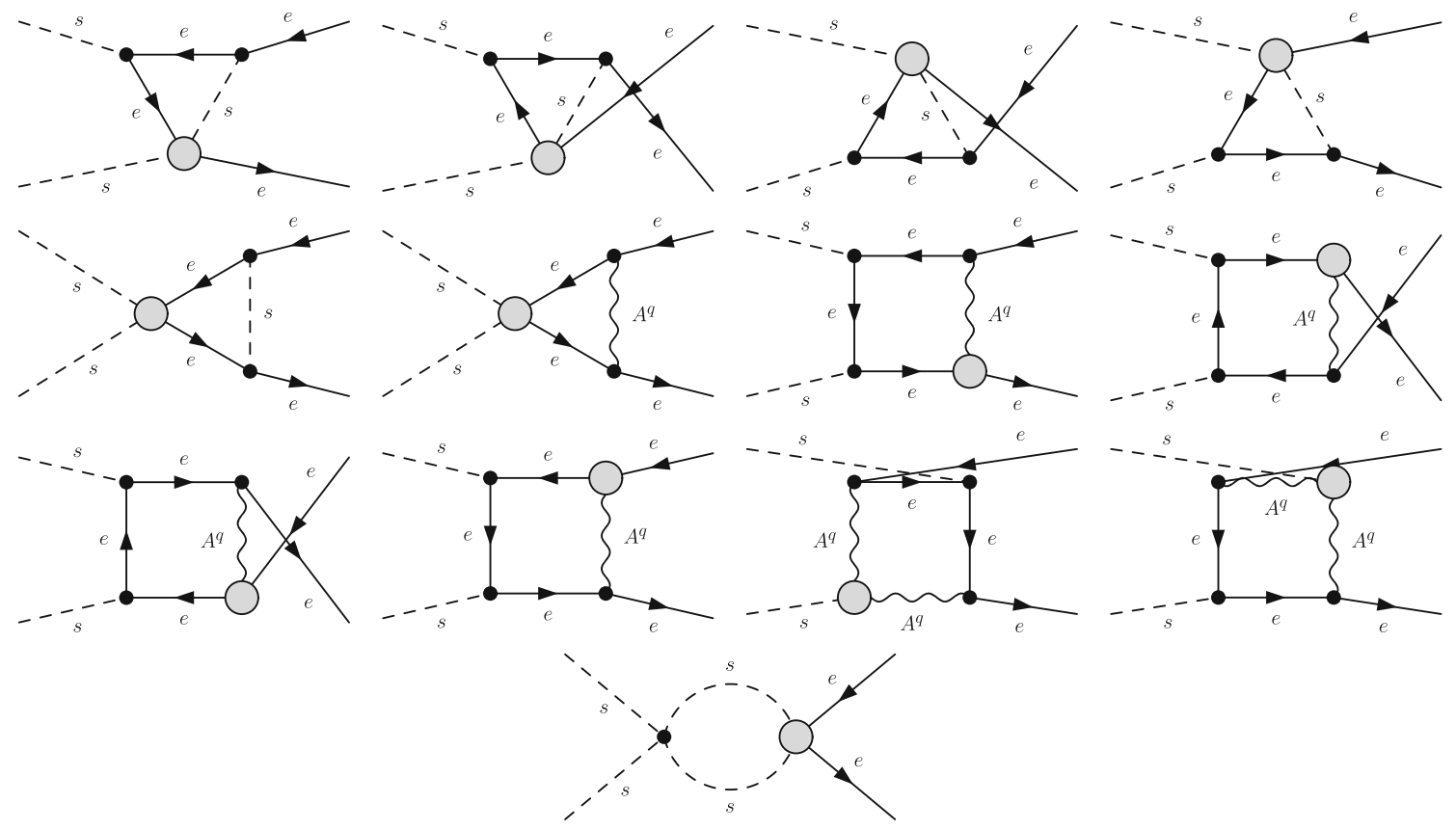

Fig. 27 Feynman diagrams for $s\left(p_{1}\right) s\left(p_{2}\right) \rightarrow \bar{e}\left(p_{3}\right) e\left(p_{4}\right)$ 


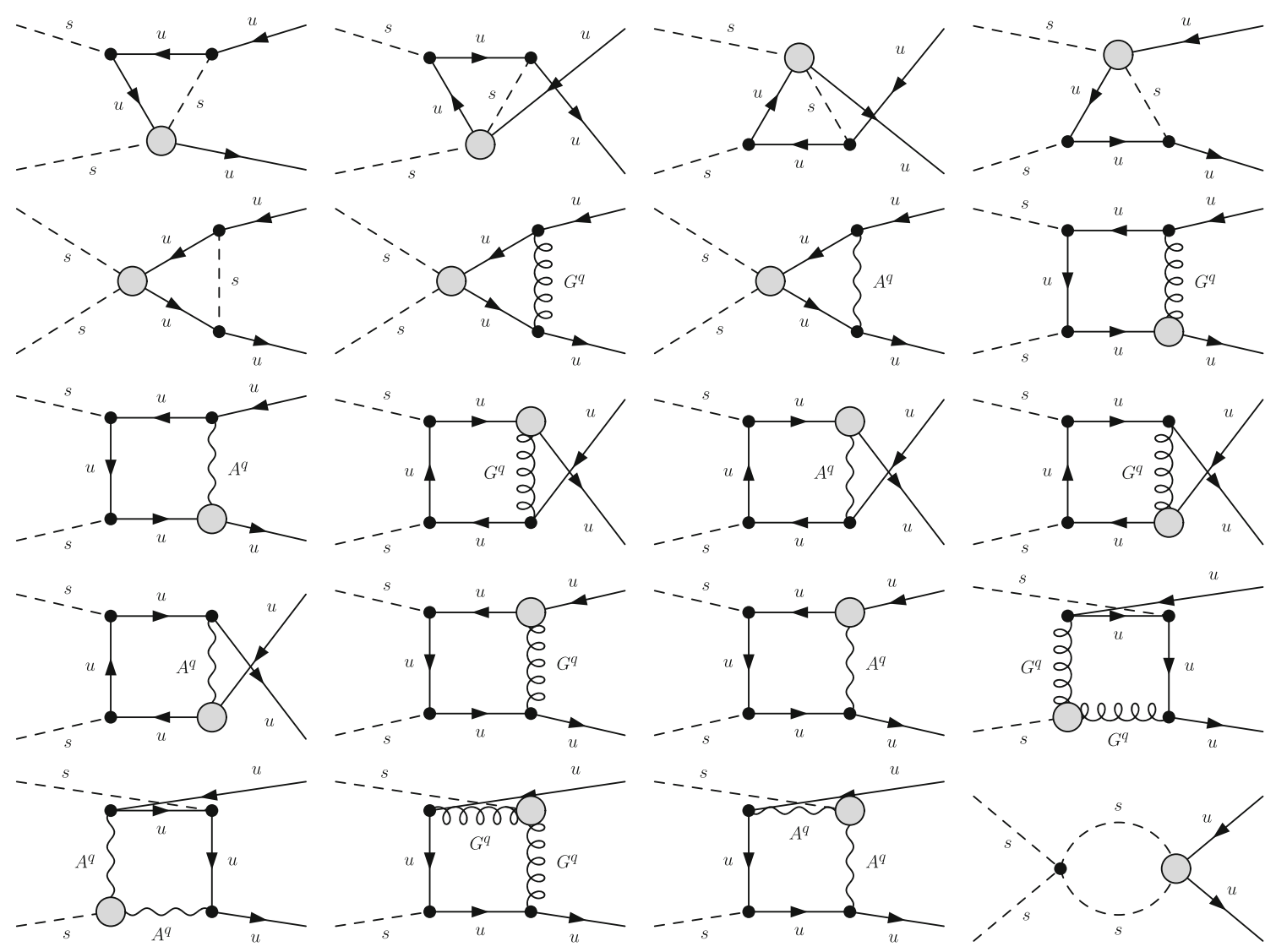

Fig. 28 Feynman diagrams for $s\left(p_{1}\right) s\left(p_{2}\right) \rightarrow \bar{u}\left(p_{3}\right) u\left(p_{4}\right)$ 

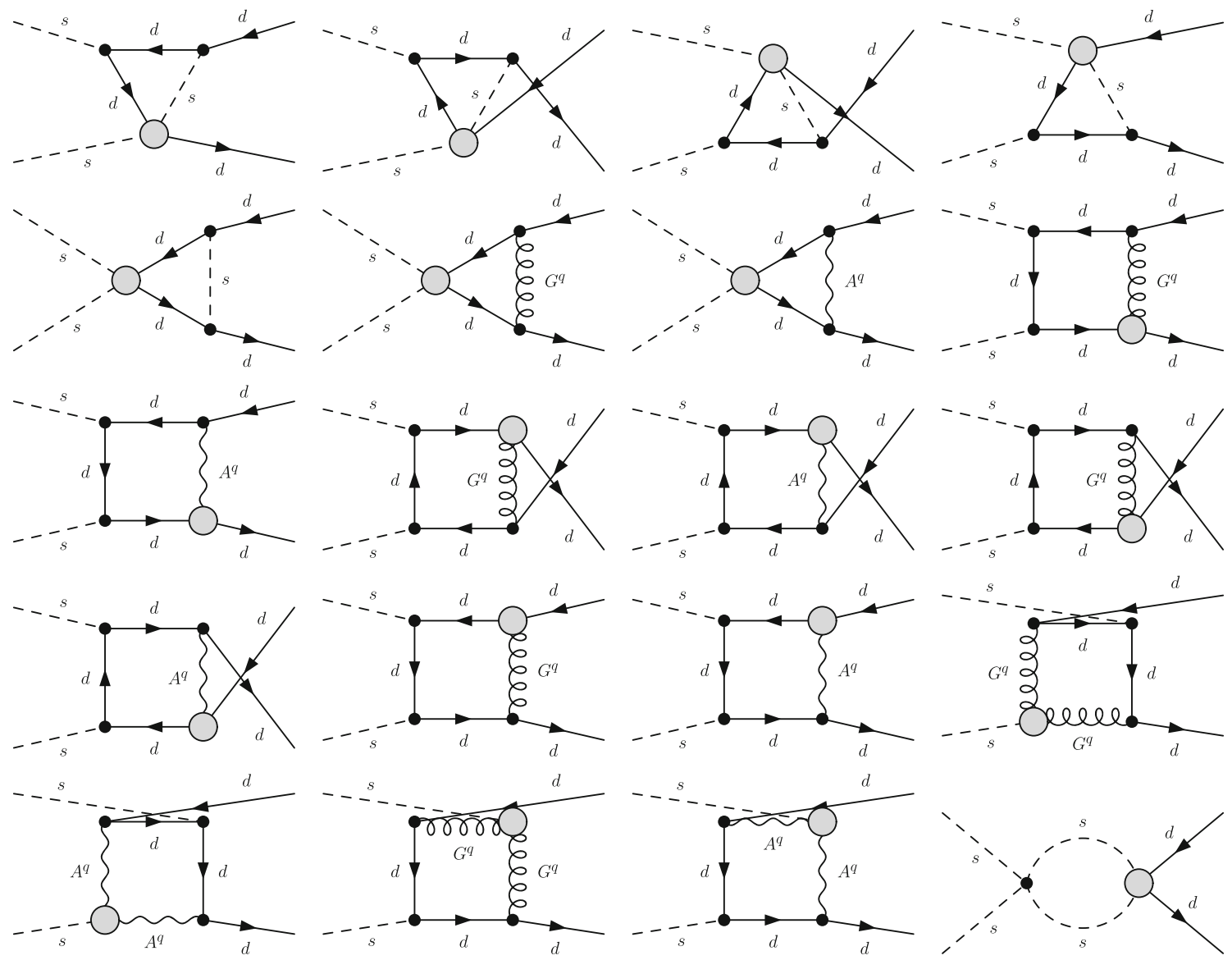

Fig. 29 Feynman diagrams for $s\left(p_{1}\right) s\left(p_{2}\right) \rightarrow \bar{d}\left(p_{3}\right) d\left(p_{4}\right)$
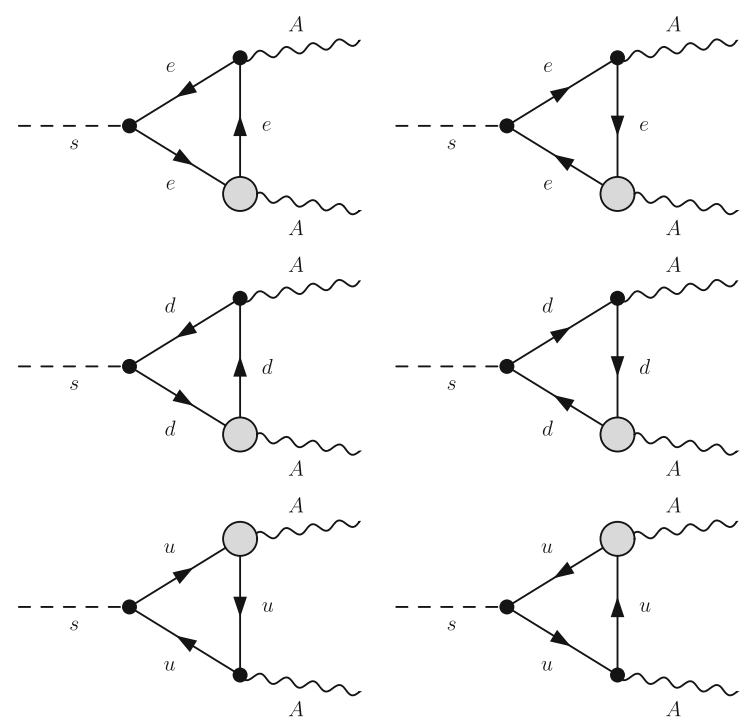

Fig. 30 Feynman diagrams for $s\left(p_{1}\right) \rightarrow A\left(p_{2}\right) A\left(p_{3}\right)$
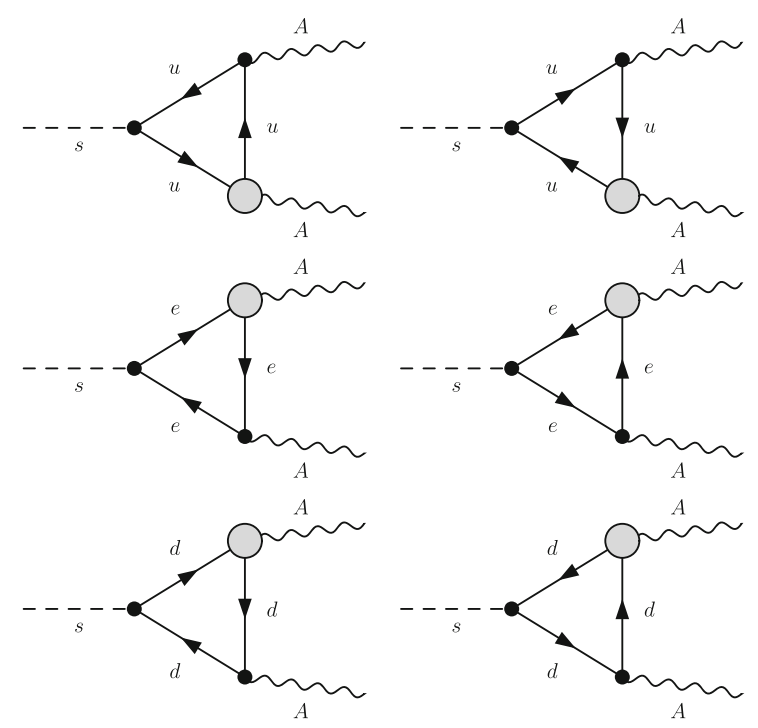

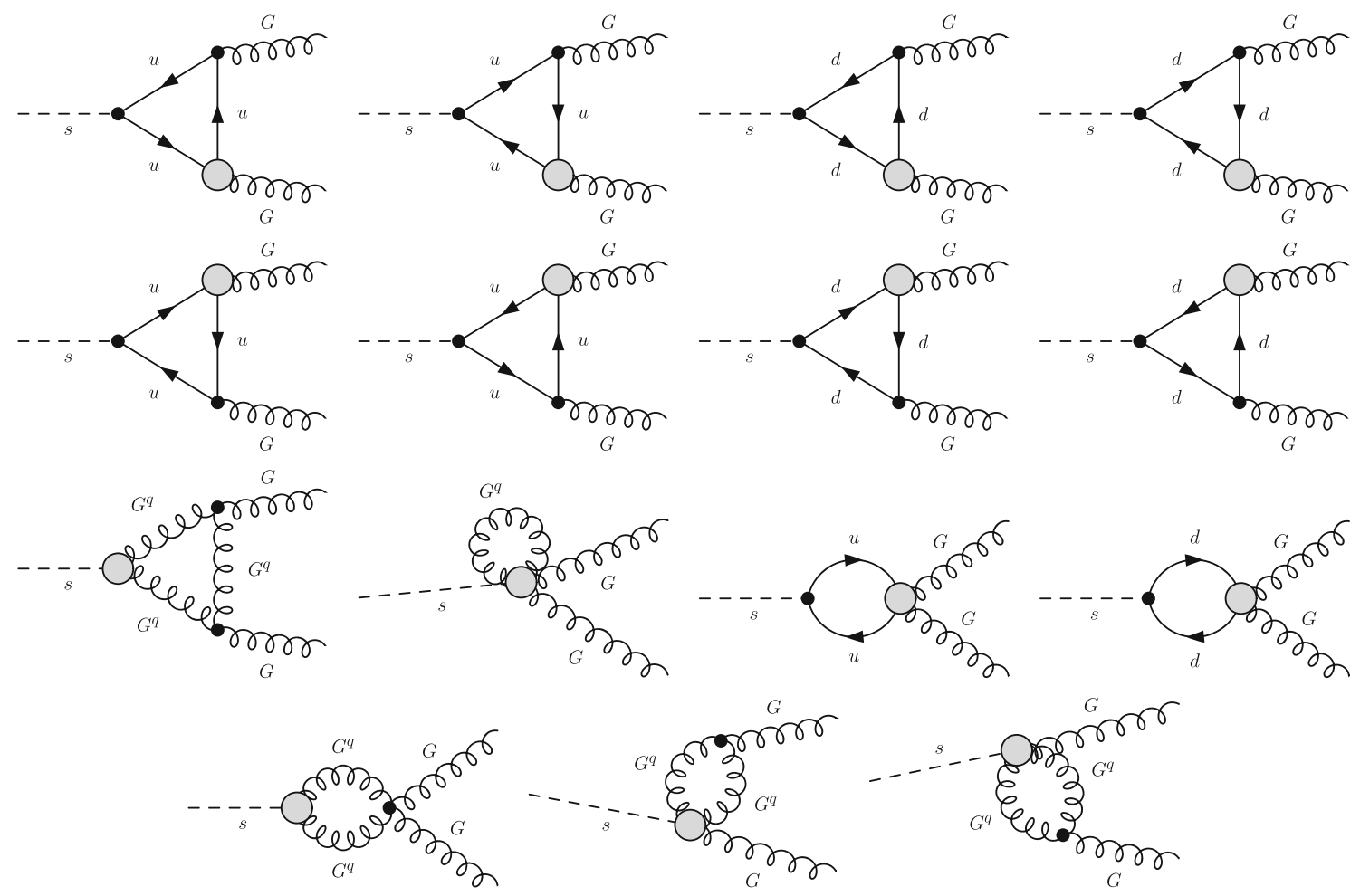

Fig. 31 Feynman diagrams for $s\left(p_{1}\right) \rightarrow G\left(p_{2}\right) G\left(p_{3}\right)$ 


\section{References}

1. R. Peccei, H.R. Quinn, CP conservation in the presence of instantons. Phys. Rev. Lett. 38, 1440 (1977). https://doi.org/10.1103/ PhysRevLett.38.1440

2. R. Peccei, H.R. Quinn, Constraints imposed by CP conservation in the presence of instantons. Phys. Rev. D 16, 1791 (1977). https:// doi.org/10.1103/PhysRevD.16.1791

3. S. Weinberg, A new light boson? Phys. Rev. Lett. 40, 223 (1978). https://doi.org/10.1103/PhysRevLett.40.223

4. F. Wilczek, Problem of strong $P$ and $T$ invariance in the presence of instantons. Phys. Rev. Lett. 40, 279 (1978). https://doi.org/10. 1103/PhysRevLett.40.279

5. B. Gripaios, A. Pomarol, F. Riva, J. Serra, Beyond the minimal composite Higgs model. JHEP 04, 070 (2009). https://doi.org/10. 1088/1126-6708/2009/04/070. arXiv:0902.1483

6. B. Gripaios, M. Nardecchia, T. You, On the structure of anomalous composite Higgs models. Eur. Phys. J. C 77, 28 (2017). https://doi. org/10.1140/epjc/s10052-017-4603-5. arXiv:1605.09647

7. M. Chala, G. Durieux, C. Grojean, L. de Lima, O. Matsedonskyi, Minimally extended SILH. JHEP 06, 088 (2017). https://doi.org/ 10.1007/JHEP06(2017)088. arXiv:1703.10624

8. F. Wilczek, Axions and family symmetry breaking. Phys. Rev. Lett. 49, 1549 (1982). https://doi.org/10.1103/PhysRevLett.49.1549

9. Y. Chikashige, R.N. Mohapatra, R. Peccei, Are there real goldstone bosons associated with broken lepton number? Phys. Lett. B 98, 265 (1981). https://doi.org/10.1016/0370-2693(81)90011-3

10. J. Preskill, M.B. Wise, F. Wilczek, Cosmology of the invisible axion. Phys. Lett. B 120, 127 (1983). https://doi.org/10.1016/ 0370-2693(83)90637-8

11. L. Abbott, P. Sikivie, A cosmological bound on the invisible axion. Phys. Lett. B 120, 133 (1983). https://doi.org/10.1016/ 0370-2693(83)90638-X

12. M. Dine, W. Fischler, The not so harmless axion. Phys. Lett. B 120, 137 (1983). https://doi.org/10.1016/0370-2693(83)90639-1

13. A. Davidson, K.C. Wali, Minimal flavour unification via multigenerational Peccei-Quinn symmetry. Phys. Rev. Lett. 48, 11 (1982). https://doi.org/10.1103/PhysRevLett.48.11

14. Y. Ema, K. Hamaguchi, T. Moroi, K. Nakayama, Flaxion: a minimal extension to solve puzzles in the standard model. JHEP 01, 096 (2017). https://doi.org/10.1007/JHEP01(2017)096. arXiv: 1612.05492

15. L. Calibbi, F. Goertz, D. Redigolo, R. Ziegler, J. Zupan, Minimal axion model from flavor. Phys. Rev. D 95, 095009 (2017). https:// doi.org/10.1103/PhysRevD.95.095009. arXiv:1612.08040

16. P.W. Graham, D.E. Kaplan, S. Rajendran, Cosmological relaxation of the electroweak scale. Phys. Rev. Lett. 115, 221801 (2015). https://doi.org/10.1103/PhysRevLett.115.221801. arXiv:1504.07551

17. R. Povey, J. Hartnett, M. Tobar, Microwave cavity light shining through a wall optimization and experiment. Phys. Rev. D 82, 052003 (2010). https://doi.org/10.1103/PhysRevD.82.052003. arXiv: 1003.0964

18. Shape ADMX Collaboration, A search for hidden sector photons with ADMX. Phys. Rev. Lett. 105, 17180 (2010). https://doi.org/ 10.1103/PhysRevLett.105.171801. arXiv:1007.3766

19. R. Essig et al., Working group report: new light weakly coupled particles, in Community Summer Study 2013: Snowmass on the Mississippi, vol. 10 (2013). arXiv:1311.0029

20. M. Betz, F. Caspers, M. Gasior, M. Thumm, S. Rieger, First results of the CERN resonant weakly interacting sub-eV particle search (CROWS). Phys. Rev. D 88, 075014 (2013). https://doi.org/10. 1103/PhysRevD.88.075014. arXiv:1310.8098

21. J.D. Bjorken, R. Essig, P. Schuster, N. Toro, New fixed-target experiments to search for dark gauge forces. Phys. Rev. D
80, 075018 (2009). https://doi.org/10.1103/PhysRevD.80.075018. arXiv:0906.0580

22. S. Andreas, C. Niebuhr, A. Ringwald, New limits on hidden photons from past electron beam dumps. Phys. Rev. D 86, 095019 (2012). https://doi.org/10.1103/PhysRevD.86.095019. arXiv: 1209.6083

23. J. Jaeckel, M. Spannowsky, Probing $\mathrm{MeV}$ to $90 \mathrm{GeV}$ axion-like particles with LEP and LHC. Phys. Lett. B 753, 482 (2016). https:// doi.org/10.1016/j.physletb.2015.12.037. arXiv:1509.00476

24. M. Bauer, M. Neubert, A. Thamm, Collider probes of axionlike particles. JHEP 12, 044 (2017). https://doi.org/10.1007/ JHEP12(2017)044. arXiv:1708.00443

25. N. Craig, A. Hook, S. Kasko, The photophobic ALP. JHEP 09, 028 (2018). https://doi.org/10.1007/JHEP09(2018)028. arXiv: 1805.06538

26. S. Knapen, T. Lin, H.K. Lou, T. Melia, Searching for axionlike particles with ultraperipheral heavy-ion collisions. Phys. Rev. Lett. 118, 171801 (2017). https://doi.org/10.1103/PhysRevLett. 118.171801. arXiv: 1607.06083

27. I. Brivio, M. Gavela, L. Merlo, K. Mimasu, J. No, R. del Rey et al., ALPs effective field theory and collider signatures. Eur. Phys. J. C 77, 572 (2017). https://doi.org/10.1140/epjc/s10052-017-5111-3. arXiv: 1701.05379

28. M. Bauer, M. Neubert, A. Thamm, LHC as an axion factory: probing an axion explanation for $(g-2)_{\mu}$ with exotic Higgs decays. Phys. Rev. Lett. 119, 031802 (2017). https://doi.org/10. 1103/PhysRevLett.119.031802. arXiv:1704.08207

29. G. Alonso-Álvarez, M. Gavela, P. Quilez, Axion couplings to electroweak gauge bosons. Eur. Phys. J. C 79, 223 (2019). https://doi. org/10.1140/epjc/s10052-019-6732-5. arXiv:1811.05466

30. J. Ebadi, S. Khatibi, M. Mohammadi Najafabadi, New probes for axionlike particles at hadron colliders. Phys. Rev. D 100, 015016 (2019). https://doi.org/10.1103/PhysRevD.100.015016. arXiv:1901.03061

31. M. Gavela, J. No, V. Sanz, J. de Trocóniz, Nonresonant searches for axionlike particles at the LHC. Phys. Rev. Lett. 124, 051802 (2020). https://doi.org/10.1103/PhysRevLett.124.051802. arXiv: 1905.12953

32. R. Coelho, V. Goncalves, D. Martins, M. Rangel, Production of axionlike particles in $\mathrm{PbPb}$ collisions at the LHC, HELHC and FCC: a phenomenological analysis. Phys. Lett. B 806, 135512 (2020). https://doi.org/10.1016/j.physletb.2020.135512. arXiv:2002.06027

33. G. Haghighat, D.H. Raissi, M. Mohammadi Najafabadi, New collider searches for axion-like particles coupling to gluons. arXiv:2006.05302

34. V. Goncalves, W. Sauter, Exclusive axionlike particle production by gluon-induced interactions in hadronic collisions. arXiv:2006.16716

35. M. Bauer, M. Heiles, M. Neubert, A. Thamm, Axion-like particles at future colliders. Eur. Phys. J. C 79, 74 (2019). https://doi.org/ 10.1140/epjc/s10052-019-6587-9. arXiv:1808.10323

36. C.-X. Yue, M.-Z. Liu, Y.-C. Guo, Searching for axionlike particles at future ep colliders. Phys. Rev. D 100, 015020 (2019). https:// doi.org/10.1103/PhysRevD.100.015020. arXiv:1904.10657

37. S. İnan, A. Kisselev, A search for axion-like particles in light-bylight scattering at the CLIC. JHEP 06, 183 (2020). https://doi.org/ 10.1007/JHEP06(2020)183. arXiv:2003.01978

38. J.L. Feng, I. Galon, F. Kling, S. Trojanowski, Axionlike particles at FASER: the LHC as a photon beam dump. Phys. Rev. D 98, 055021 (2018). https://doi.org/10.1103/PhysRevD.98.055021. arXiv: 1806.02348

39. Shape FASER Collaboration, FASER's physics reach for longlived particles. Phys. Rev. D 99, 095011 (2019). https://doi.org/ 10.1103/PhysRevD.99.095011. arXiv: 1811.12522 
40. F. Kling, S. Trojanowski, Looking forward to test the KOTO anomaly with FASER. Phys. Rev. D 102, 015032 (2020). https:// doi.org/10.1103/PhysRevD.102.015032. arXiv:2006.10630

41. Shape CAST collaboration, CAST search for sub-eV mass solar axions with $3 \mathrm{He}$ buffer gas. Phys. Rev. Lett. 107, 261302 (2011). https://doi.org/10.1103/PhysRevLett.107.261302. arXiv: 1106.3919

42. G.G. Raffelt, Astrophysical axion bounds. Lect. Notes Phys. 741, 51 (2008). https://doi.org/10.1007/978-3-540-73518-2_3. arXiv:hep-ph/0611350

43. J.S. Lee, Revisiting supernova 1987A limits on axion-likeparticles. arXiv:1808.10136

44. J.H. Chang, R. Essig, S.D. McDermott, Supernova 1987A constraints on sub-GeV dark sectors. Millicharged particles, the QCD axion, and an axion-like particle. JHEP 09, 051 (2018). https://doi. org/10.1007/JHEP09(2018)051. arXiv:1803.00993

45. J. Jaeckel, L.J. Thormaehlen, Axions as a probe of solar metals. Phys. Rev. D 100, 123020 (2019). https://doi.org/10.1103/ PhysRevD.100.123020. arXiv:1908.10878

46. P. Carenza, T. Fischer, M. Giannotti, G. Guo, G. Martínez-Pinedo, A. Mirizzi, Improved axion emissivity from a supernova via nucleon-nucleon bremsstrahlung. JCAP 10, 016 (2019). https:// doi.org/10.1088/1475-7516/2019/10/016. arXiv:1906.11844

47. F. Ertas, F. Kahlhoefer, On the interplay between astrophysical and laboratory probes of $\mathrm{MeV}$-scale axion-like particles. JHEP 07, 050 (2020). https://doi.org/10.1007/JHEP07(2020)050. arXiv:2004.01193

48. G. Lucente, P. Carenza, T. Fischer, M. Giannotti, A. Mirizzi, Heavy axion-like particles and core-collapse supernovae: constraints and impact on the explosion mechanism. JCAP 12, 008 (2020). https:// doi.org/10.1088/1475-7516/2020/12/008. arXiv:2008.04918

49. P. Carenza, B. Fore, M. Giannotti, A. Mirizzi, S. Reddy, Enhanced supernova axion emission and its implications. arXiv:2010.02943

50. W. Marciano, A. Masiero, P. Paradisi, M. Passera, Contributions of axionlike particles to lepton dipole moments. Phys. Rev. D 94, 115033 (2016). https://doi.org/10.1103/PhysRevD.94.115033. arXiv: 1607.01022

51. M. Gavela, R. Houtz, P. Quilez, R. Del Rey, O. Sumensari, Flavor constraints on electroweak ALP couplings. Eur. Phys. J. C 79, 369 (2019). https://doi.org/10.1140/epjc/s10052-019-6889-y. arXiv: 1901.02031

52. M. Bauer, M. Neubert, S. Renner, M. Schnubel, A. Thamm, Axionlike particles, lepton-flavor violation, and a new explanation of $a_{\mu}$ and $a_{e}$. Phys. Rev. Lett. 124, 211803 (2020). https://doi.org/10. 1103/PhysRevLett.124.211803. arXiv:1908.00008

53. C. Cornella, P. Paradisi, O. Sumensari, Hunting for ALPs with lepton flavor violation. JHEP 01, 158 (2020). https://doi.org/10. 1007/JHEP01(2020)158. arXiv:1911.06279

54. L. Calibbi, D. Redigolo, R. Ziegler, J. Zupan, Looking forward to lepton-flavor-violating ALPs. arXiv:2006.04795

55. J. Martin Camalich, M. Pospelov, P.N.H. Vuong, R. Ziegler, J. Zupan, Quark flavor phenomenology of the QCD axion. Phys. Rev. D 102, 015023 (2020). https://doi.org/10.1103/PhysRevD. 102.015023. arXiv:2002.04623

56. L. Di Luzio, R. Grober, P. Paradisi, Hunting for the CP violating ALP. arXiv:2010.13760

57. L. Di Luzio, M. Giannotti, E. Nardi, L. Visinelli, The landscape of QCD axion models. Phys. Rep. 870, 1 (2020). https://doi.org/10. 1016/j.physrep.2020.06.002. arXiv:2003.01100

58. K. Choi, S.H. Im, C.S. Shin, Recent progresses in physics of axions or axion-like particles. arXiv:2012.05029

59. M. Bauer, C. Hörner, M. Neubert, Diphoton resonance from a warped extra dimension. JHEP 07, 094 (2016). https://doi.org/10. 1007/JHEP07(2016)094. arXiv:1603.05978
60. K. Choi, S.H. Im, C.B. Park, S. Yun, Minimal flavor violation with axion-like particles. JHEP 11, 070 (2017). https://doi.org/10.1007/ JHEP11(2017)070. arXiv:1708.00021

61. B. Grzadkowski, M. Iskrzynski, M. Misiak, J. Rosiek, Dimensionsix terms in the standard model Lagrangian. JHEP 10, 085 (2010). https://doi.org/10.1007/JHEP10(2010)085. arXiv:1008.4884

62. J.C. Criado, BasisGen: automatic generation of operator bases. Eur. Phys. J. C 79, 256 (2019). https://doi.org/10.1140/epjc/ s10052-019-6769-5. arXiv:1901.03501

63. H. Georgi, D.B. Kaplan, L. Randall, Manifesting the invisible axion at low-energies. Phys. Lett. B 169, 73 (1986). https://doi.org/10. 1016/0370-2693(86)90688-X

64. A. Alloul, N.D. Christensen, C. Degrande, C. Duhr, B. Fuks, FeynRules 2.0 - a complete toolbox for tree-level phenomenology. Comput. Phys. Commun. 185, 2250 (2014). https://doi.org/ 10.1016/j.cpc.2014.04.012. arXiv:1310.1921

65. T. Hahn, Generating Feynman diagrams and amplitudes with FeynArts 3. Comput. Phys. Commun. 140, 418 (2001). https://doi.org/ 10.1016/S0010-4655(01)00290-9. arXiv:hep-ph/0012260

66. T. Hahn, M. Perez-Victoria, Automatized one loop calculations in four-dimensions and D-dimensions. Comput. Phys. Commun. 118, 153 (1999). https://doi.org/10.1016/ S0010-4655(98)00173-8. arXiv:hep-ph/9807565

67. P. Nogueira, Automatic Feynman graph generation. J. Comput. Phys. 105, 279 (1993). https://doi.org/10.1006/jcph.1993.1074

68. C. Cheung, C.-H. Shen, Nonrenormalization theorems without supersymmetry. Phys. Rev. Lett. 115, 071601 (2015). https://doi. org/10.1103/PhysRevLett.115.071601. arXiv:1505.01844

69. Z. Bern, J. Parra-Martinez, E. Sawyer, Nonrenormalization and operator mixing via on-shell methods. Phys. Rev. Lett. 124, 051601 (2020). https://doi.org/10.1103/PhysRevLett.124.051601. arXiv: 1910.05831

70. G. Buchalla, A. Celis, C. Krause, J.-N. Toelstede, Master formula for one-loop renormalization of bosonic SMEFT operators. arXiv:1904.07840

71. M. Chala, A. Titov, One-loop running of dimension-six Higgsneutrino operators and implications of a large neutrino dipole moment. arXiv:2006.14596

72. F. Lyonnet, I. Schienbein, PyR@TE 2: a Python tool for computing RGEs at two-loop. Comput. Phys. Commun. 213, 181 (2017). https://doi.org/10.1016/j.cpc.2016.12.003. arXiv:1608.07274

73. E.E. Jenkins, A.V. Manohar, P. Stoffer, Low-energy effective field theory below the electroweak scale: anomalous dimensions. JHEP 01, 084 (2018). https://doi.org/10.1007/JHEP01(2018)084. arXiv: 1711.05270

74. E.E. Jenkins, A.V. Manohar, P. Stoffer, Low-energy effective field theory below the electroweak scale: operators and matching. JHEP 03, 016 (2018). https://doi.org/10.1007/JHEP03(2018)016. arXiv: 1709.04486

75. J. Terol-Calvo, M. Tórtola, A. Vicente, High-energy constraints from low-energy neutrino nonstandard interactions. Phys. Rev. D 101, 095010 (2020). https://doi.org/10.1103/PhysRevD.101. 095010. arXiv:1912.09131

76. J.C. Collins, A.V. Manohar, M.B. Wise, Renormalization of the vector current in QED. Phys. Rev. D 73, 105019 (2006). https:// doi.org/10.1103/PhysRevD.73.105019. arXiv:hep-th/0512187 